\title{
Software Safety Hazard Analysis
}

\author{
AECLITEL

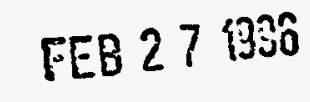 \\ OSTI
}

Prepared by

J. D. Lawrence

Lawrence Livermore National Laboratory

Prepared for

U.S. Nuclear Regulatory Commission 


\section{AVAILABILITY NOTICE}

Availability of Reference Materials Cited in NRC Publications

Most documents cited in NRC publications will be available from one of the following sources:

1. The NRC Public Document Room, 2120 L Street, NW., Lower Level, Washington, DC 20555-0001

2. The Superintendent of Documents, U.S. Government Printing Office, P. O. Box 37082 , Washington, DC 20402-9328

3. The National Technical Information Service, Springfield, VA 22161-0002

Although the listing that follows represents the majority of documents cited in NRC publications, it is not intended to be exhaustive.

Referenced documents available for inspection and copying for a fee from the NRC Public Document Room include NRC correspondence and internal NRC memoranda; NRC bulletins, circulars, information notices, inspection and investigation notices; licensee event reports; vendor reports and correspondence; Commission papers; and applicant and licensee documents and correspondence.

The following documents in the NUREG series are available for purchase from the Government Printing Office: formal NRC staff and contractor reports, NRC-sponsored conference proceedings, international agreement reports, grantee reports, and NRC booklets and brochures. Also available are regulatory guides, NRC regulations In the Cocte of Federal Regulations, and Nuclear Regulatory Commission Issuances.

Documents avallable from the National Technical Information Service include NUREG-series reports and technical reports prepared by other Federal agencies and reports prepared by the Atomic Energy Commission, forerunner agency to the Nuclear Regulatory Commission.

Documents available from public and special technical libraries include all open literature items, such as books, Journal articles, and transactions. Federal Register notices. Federal and State legislation, and congressional reports can usually be obtained from these libraries.

Documents such as theses, dissertations, foreign reports and translations, and non-NRC conference proceedings are available for purchase from the organization sponsoring the publication cited.

Single coples of NRC draft reports are available free. to the extent of supply. upon written request to the Office of Administration, Distribution and Mail Services Section, U.S. Nuclear Regulatory Commission, Washington, DC 20555-0001.

Coples of industry codes and standards used in a substantive manner in the NRC regulatory process are maintained at the NRC Library. Two White Flint North, 11545 Rockville Pike, Rockville. MD 20852-2738, for use by the public. Codes and standards are usually copyrighted and may be purchased from the originating organization or, if they are American National Standards, from the American National Standards Institute, 1430 Broadway, New York, NY 10018-3308.

\section{DISCLAIMER NOTICE}

This report was prepared as an account of work sponsored by an agency of the United States Government. Neither the United States Government nor any agency thereof, nor any of their employees, makes any warranty, expressed or implied, or assumes any legal liability or responsibility for any third party's use, or the results of such use, of any information, apparatus, product, or process disclosed in this report, or represents that its use by such third party would not infringe privately owned rights. 
NUREG/CR-6430

UCRL-ID-122514

\section{Software Safety Hazard Analysis}

Manuscript Completed: October 1995

Date Published: February 1996

Prepared by

J. D. Lawrence

Lawrence Livermore National Laboratory

7000 East Avenue

Livermore, CA 94550

J. Gallagher, NRC Technical Monitor

Prepared for

Division of Reactor Controls and Human Factors

Office of Nuclear Reactor Regulation

U.S. Nuclear Regulatory Commission

Washington, DC 20555-0001

NRC Job Code L1867 


\section{.}

. 


\begin{abstract}
Techniques for analyzing the safety and reliability of analog-based electronic protection systems that serve to mitigate hazards in process control systems have been developed over many years, and are reasonably well understood. An example is the protection system in a nuclear power plant The extension of these techniques to systems which include digital computers is not well developed, and there is little consensus among software engineering experts and safety experts on how to analyze such systems.

One possible technique is to extend hazard analysis to include digital computer-based systems. Software is frequently overlooked during system hazard analyses, but this is unacceptable when the software is in control of a potentially hazardous operation. In such cases, hazard analysis should be extended to fully cover the software. A method for performing software hazard analysis is proposed in this paper.
\end{abstract}





\section{CONTENTS}

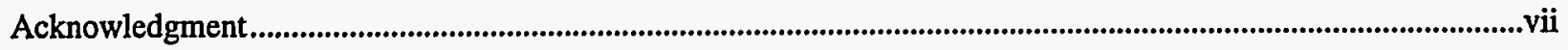

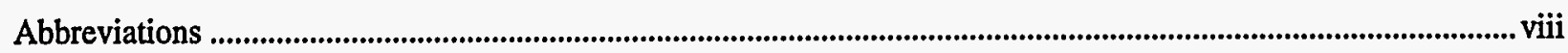

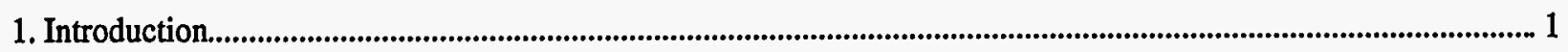

1.1. Purpose ................................................................................................................................................................ 1

1.2. Report Structure ........................................................................................................................................ 1

1.3. Terminology ....................................................................................................................................................... 2

2. Introduction to the Software Hazard Analysis Process .................................................................................................3

2.1. Software Hazard Analysis as Part of System Safety Analysis................................................................................. 3

2.2. Software Hazard Analysis as Part of Software Design ....................................................................................4

2.3. General Approach to Software Hazard Analysis .............................................................................................. 4

2.4. Prerequisites to Software Hazard Analysis ...............................................................................................................5

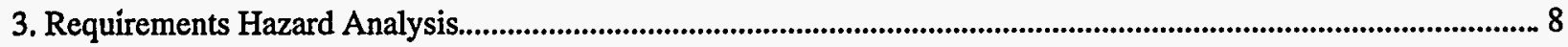

3.1. Inputs to Software Requirements Hazard Analysis......................................................................................... 14

3.2. Analysis Procedures ................................................................................................................................................ 14

3.3. Outputs of Software Requirements Hazard Analysis................................................................................. 15

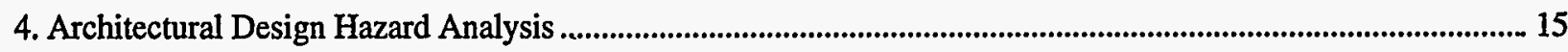

4.1. Inputs to Software Architecture Hazard Analysis ...................................................................................................16

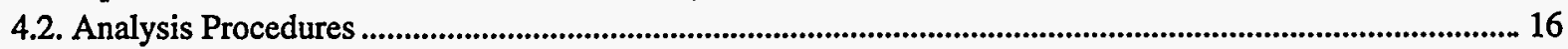

4.3. Outputs of Software Architecture Hazard Analysis .................................................................................... 18

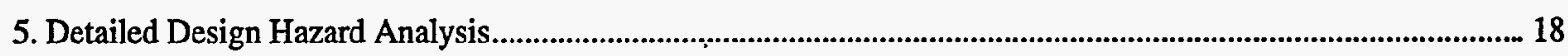

5.1. Inputs to Software Detailed Design Hazard Analysis ................................................................................19

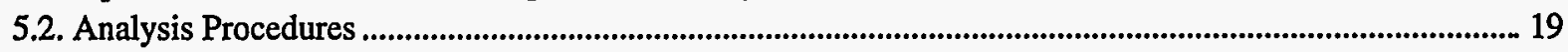

5.3. Outputs of Software Detailed Design Hazard Analysis ...................................................................................... 19

6. Code Hazard Analysis .......................................................................................................................................................19

6.1. Inputs to Software Code Hazard Analysis ............................................................................................................ 20

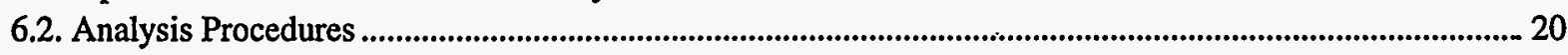

6.3. Outputs of Software Code Hazard Analysis.................................................................................................. 20

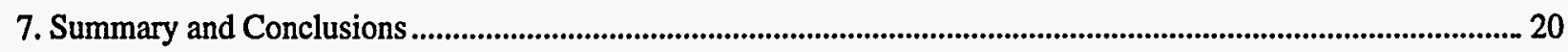

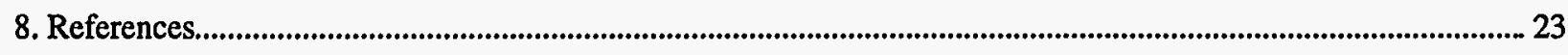

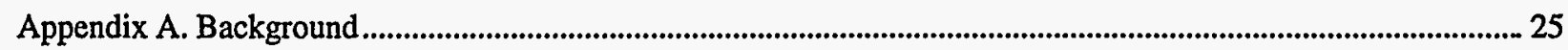

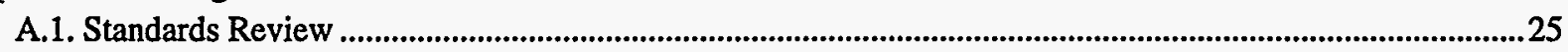

A.2. General Discussion of Hazard Analysis ........................................................................................................ 33

A.3. NIST Review of Software Hazard Analyses.................................................................................... 38

A.4. Review of the Published Literature ........................................................................................................ 39

Appendix B. Potential Software Safety Analysis Methods ..........................................................................................4

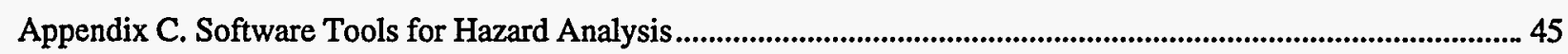

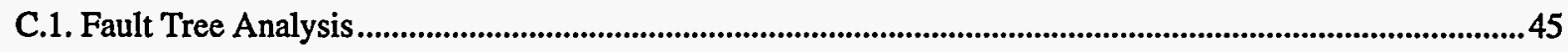

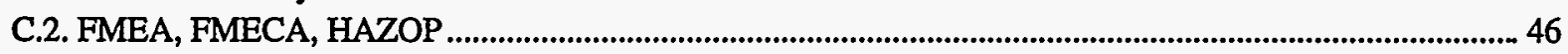

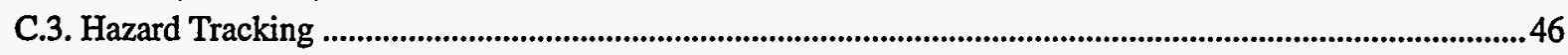

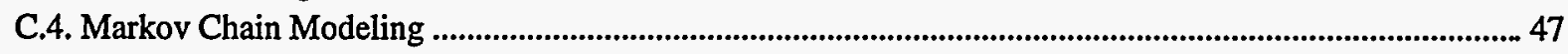

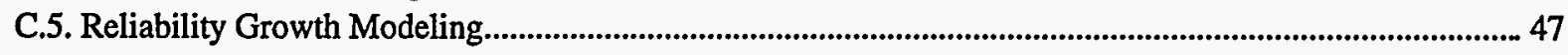




\section{FIGURES}

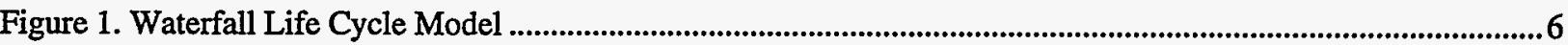

Figure 2. Software Hazard Analysis within the Software Life Cycle ..........................................................................7

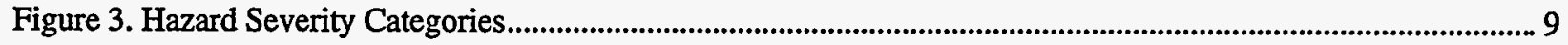

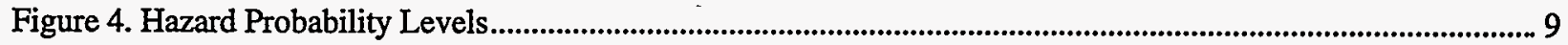

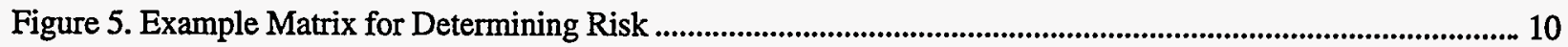

Figure 6. Software Qualities Relating to Potential Hazards.................................................................................... 10

Figure 7. Guide Phrases for Software Qualities ................................................................................................. 11

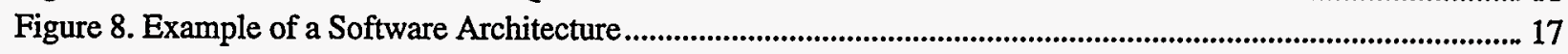

Figure 9. Determination of Architecture Risk Levels ......................................................................................18

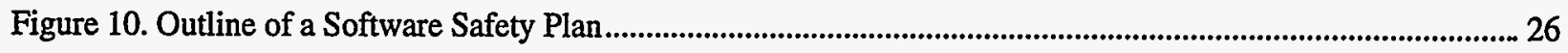

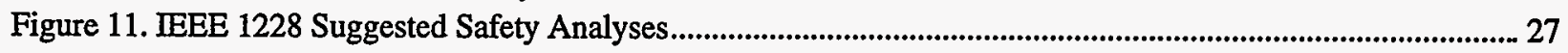

Figure 12. Hazard Severity Categories (from Mil-Std 882C) ................................................................................28

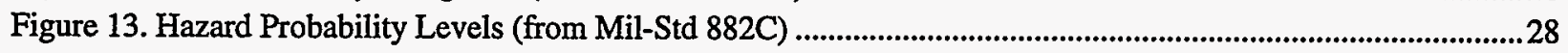

Figure 14. Detailed Safety Tasks (from Mil-Std 882C) .......................................................................................... 29

Figure 15. Example Matrix for Residual Risk (from Mil-Std 882C) ........................................................................... 29

Figure 16. Example Software Hazard Criticality Matrix (from Mil-Std 882C).......................................................... 31

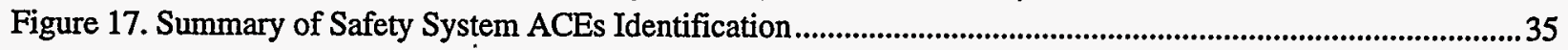

Figure 18. Summary of Software Requirements ACEs Identification ..................................................................... 35

Figure 19. Summary of Software Design ACEs Identification ......................................................................................36

Figure 20. Summary of Software Code ACEs Identification...................................................................................... 36

Figure 21. Summary of General Guidelines for ACE Resolution ............................................................................37

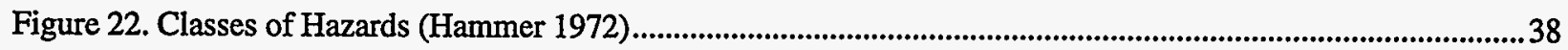

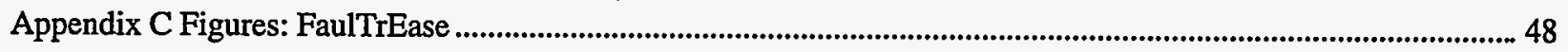

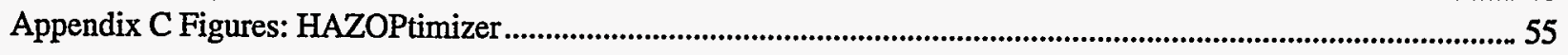

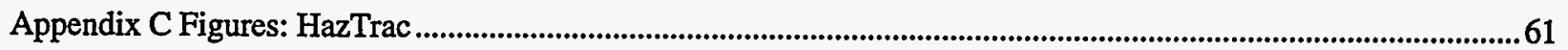

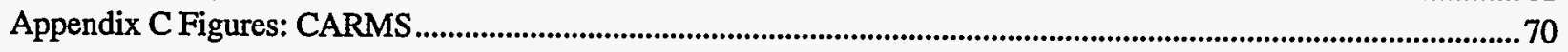

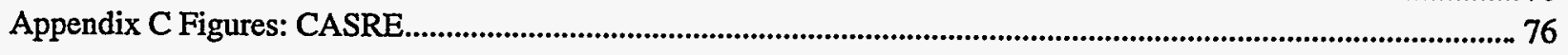




\section{ACKNOWLEDGMENT}

The author thanks and acknowledges the efforts of Mr. John Gallagher from the Nuclear Regulatory Commission who reviewed this work and provided insights and comments. 


\begin{tabular}{ll} 
& \multicolumn{1}{c}{ ABBREVIATIONS } \\
ACE & Abnormal Condition or Event \\
CHA & Component Hazard Analysis \\
COTS & Commercial Off-the-Shelf \\
DOD & Department of Defense \\
ETA & Event Tree Analysis \\
FMEA & Failure Modes and Effects Analysis \\
FMECA & Failure Modes, Effects and Criticality Analysis \\
FSE & Functions, Systems and Equipment \\
FTA & Fault Tree Analysis \\
HAZOP & Hazard and Operability Analysis \\
I\&C & Instrumentation and Control \\
IEEE & Institute of Electronic and Electrical Engineers \\
MOD & Ministry of Defense \\
NPP & Nuclear Power Plant \\
NSCCA & Nuclear Safety Cross-Check Analysis \\
O\&SHA & Operating and Support Hazard Analysis \\
PHA & Preliminary Hazard Analysis \\
PHL & Preliminary Hazard List \\
PIE & Postulated Initiating Event \\
PRA & Probabilistic Risk Assessment \\
RPS & Reactor Protection System \\
SAD & Software Architecture Description \\
SDD & Software Design Description \\
Software Hazard Analysis \\
Softem Hazard Analysis \\
Software Requirements Specification \\
SHA
\end{tabular}




\section{SOFTWARE SAFETY HAZARD ANALYSIS}

\section{INTRODUCTION}

\subsection{Purpose}

Techniques for analyzing the safety and reliability of analog-based electronic protection systems that serve to mitigate hazards in process control systems have been developed over many years, and are reasonably well understood. An example is the protection system in a nuclear power plant. The extension of these techniques to systems which include digital computers is not well developed, and there is little consensus among software engineering experts and safety experts on how to analyze such systems.

One possible technique is to extend hazard analysis to include digital computer-based systems. If safety is considered to be a measure of the degree of freedom from risk, then software safety ensures that the software in the computer system will execute within the application system without resulting in unacceptable risk. Hazard analysis is a method of identifying portions of a system which have the potential for unacceptable hazards; the purpose is to (1) encourage design changes which will reduce or eliminate hazards, or (2) carry out special analyses and tests which can provide increased confidence in especially vulnerable portions of the system.

Software is frequently overlooked during system hazard analyses, but this is unacceptable when the software is in control of a potentially hazardous operation. In such cases, hazard analysis should be extended to fully cover the software. A method for performing software hazard analysis is proposed in this paper. ${ }^{1}$

The report considers only those hazards affected by software. Only the software portion of the digital computer system is considered. In

1 Neither this proposed method of hazard analysis nor any other specific method has been endorsed by the U. S. Nuclear Regulatory Commission. particular, it is assumed that the computer hardware operates without failure. ${ }^{2}$

As a consequence of the above assumptions, the report concentrates on two questions.

- If the software operates correctly (i.e., follows its specifications), what is the potential effect on system hazards?

- If the software operates incorrectly (i.e., deviates from specifications), what is the potential effect on system hazards?

This report does not discuss how to determine whether a software item is correct or not. Software analyses, reviews and tests directed at finding faults in the software are not considered to be a direct part of software hazard analysis. See Lawrence (1993) for a discussion of these Verification and Validation (V\&V) topics within the software life cycle.

Although V\&V is not considered to be part of hazard analysis, the results of a V\&V effort may well be of use. For example, the use of testing to estimate the reliability of a software item might be used within a fault tree analysis to estimate the probability of a hazard occurring.

The performance of software hazard analysis can be facilitated by the use of automated or semi-automated tools. Examples of such tools are considered in Appendix $\mathbf{C}$.

\subsection{Report Structure}

Software hazard analysis is discussed in general terms in Chapter 2. This chapter includes a list of desirable prerequisites to software hazard analysis, and a general discussion of the approach proposed in the remainder of the report.

Chapters 3-6 provide the details of the proposed software hazard analysis process. Considerable emphasis is placed on the requirements and

\footnotetext{
2 A separate hardware hazard analysis and-for complex computer systems-a separate computer system hazard analysis, are recommended to supplement the software hazard analysis.
} 
architecture design phases of the software life cycle to reflect the belief that faults in requirements and architecture design specifications have a greater potential impact on system hazards than faults in the detailed design or coding phases.

Tool support can be very helpful when performing hazard analyses. A representative set of tools is discussed briefly in Appendix C. The goal here is to indicate the availability of different types of tools. The tools were selected for discussion based on availability on a PC platform and on price. No endorsement of specific tools is implied.

The software hazard analysis process proposed in this report is based on a variety of standards and technical papers described in Appendix A. The report continues with a list of possible safety analysis techniques taken from a System Safety Society report (Appendix B).

\subsection{Terminology}

Safety engineering has special terminology of its own. The following definitions, based primarily on those contained in IEEE Standard 1228, are used in this report. They are reasonably standard definitions that have been specialized to computer software in a few places.

- An accident is an unplanned event or series of events that result in death, injury, illness, environmental damage, or damage to or loss of equipment or property. (The word mishap is sometimes used to mean an accident, financial loss or public relations loss.)

Accidents generally can be divided into two categories: those that involve the unplanned release of energy and those that involve the unplanned release of toxicity.
- A system hazard is an application system condition that is a prerequisite to an accident.

That is, the system states can be divided into two sets. No state in the first set (of nonhazardous states) can directly lead to an accident, while accidents may result from any state in the second set (of hazardous states). Note that a system can be in a hazardous state without an accident occurring. It is the potential for causing an accident that creates the hazard, not necessarily the actuality, because conditions that convert the hazard to an accident are not concurrently present. A hazard is a potential for an accident that can be converted to actuality by encountering a triggering event or condition within the foreseeable operational envelope of the system.

- The term risk is used to designate a measure that combines the likelihood that a system hazard will occur, the likelihood that an accident will occur and an estimate of the severity of the worst plausible accident.

The simplest measure is to simply multiply the probability that a hazard occurs, the conditional probability that a triggering event or condition will occur while the hazard is present, and the estimated worstcase severity of the accident.

- Safety-critical software ${ }^{3}$ is software whose inadvertent response to stimuli, failure to respond when required, response out-ofsequence, or response in unplanned combination with others can result in an accident or the exacerbation of an accident. This includes software whose operation or failure to operate can lead to a hazardous state, software intended to recover from equipment malfunctions or

\footnotetext{
${ }^{3}$ The word "critical," as used in this report, refers to software criticality, not nuclear criticality.
} 
external insults, and software intended to mitigate the severity of, or recover from, an accident. 4

- A critical system is a system whose failure may lead to unacceptable consequences. The results of failure may affect the developers of the system, its direct users, their customers or the general public. The consequences may involve loss of life or property, financial loss, legal liability, regulatory actions or even the loss of good will if that is extremely important. The term safety critical refers to a system whose failure could lead to loss of life, injury, or damage to the environment. For nuclear reactors, this includes radiation releases or exposure to the public or operators and reactor workers.

- The term safety is used to mean the extent to which a system is free from system hazard.

- Hazard analysis is the process of identifying and evaluating the hazards of a system, and then either eliminating the hazard or reducing its risk to an acceptable level. (NIST 1993)

- Software hazard analysis "... eliminates or controls software hazards and hazards related to interfaces between the software and the system (including hardware and human components). It includes analyzing the requirements, design, code, user interfaces and changes." (NIST 1993)

\section{INTRODUCTION TO THE SOFTWARE HAZARD ANALYSIS PROCESS}

\subsection{Software Hazard Analysis as Part of System Safety Analysis}

Software hazard analysis should be performed within the context of the overall system design,

\footnotetext{
${ }^{4}$ There are cases in which software other than control system software can cause a hazard. An example is a fire suppression modeling program which makes incorrect recommendations for fire suppression equipment, causing a hazard in case of fire due to lack of the necessary fire fighting equipment. Such cases are outside the scope of this report.
}

for both those attributes of the system design that contribute to the system's ability to perform the assigned tasks that are derived from the plant's safety mission as well as the assigned tasks derived from the plant's primary mission that could be detrimental to the plant's safety mission. Consequently, those performing the software hazard analysis must understand the role of the software in the performance of the system safety functions and also in the performance of the system control and monitoring functions, and the effect of the software acting within the system with respect to its potential impact on the accomplishment of the plant's safety mission. This understanding is obtained from the system safety analysis; in particular, the system's hazard analysis. IEEE Standard 1228 presents the relationship between the system safety analysis and the software safety analysis in more detail. The following discussion provides an overview of the safety case for a nuclear power plant.

The safety properties of a nuclear reactor design are fundamentally affected by three broad design principles: quality, diversity and defense-indepth. These principles may be applied at various levels of the design; determining where and how to apply the principles is one of the more important activities of the design process.

All three principles should have wide applicability to other forms of process control systems.

The main hazards in a nuclear reactor are the possibility of a rapid, energetic fission reaction (e.g., Chernobyl) and the release of radioactive fission products which are the waste products of the fission reaction. In the U.S. (and many other countries), regulations mandate that the physics of the core design make rapid reactions self limiting. This leaves the prevention of the release of fission products as the main hazard to be controlled.

Three levels of defense-in-depth are provided to control the release of fission products. Each is sufficient to prevent public exposure to any significant level of radiation. First, each element of the fuel is surrounded by a barrier. In light water reactors, the fuel is composed of 
numerous metal tubes, each tube containing fuel pellets and associated fission products. Second, fission products that might be released from the fuel are further contained by the reactor coolant systems. Should some event cause breach of both of these barriers, a third barrier, the containment building, surrounds the reactor coolant system. Each of these barriers is fundamentally different in design, providing diversity at each level of defense-in-depth.

Barrier integrity is maintained first by building in a high degree of quality, and second by ensuring the barriers are not exposed to environmental conditions that exceed design assumptions. Active systems are provided to enforce these environmental limits. For example, the most important environmental considerations for the fuel are that the heat generated by the fuel be limited, and that the heat that is generated be taken away. These are the safety functions that must be accomplished to ensure the barrier (fuel clad) immediately surrounding the fuel and fission products remains intact. Diversity and defense-in-depth are provided for these functions. For example, power can be limited by dropping solid neutron absorbers (control rods) or injecting liquid absorber into the coolant system.

Each function can be actuated by multiple independent systems. For example, the control rods may be inserted automatically by the control system, the reactor protection system, the ATWS (Anticipated Transient Without Scram) Mitigation System, the Engineered Safety Actuation System (ESFAS), or the reactor operator. In proposed U. S. advanced reactor designs that involve computer-based control and protection systems, at least two diverse, automatic systems must be capable of initiating each safety function such that the consequences of each postulated accident are acceptable. Furthermore, sufficient information and manual controls must be provided to allow the operator to start and control each safety function.

This diversity may be accomplished via diversity in the computer systems. Hardware diversity may include multiple CPU types and multiple communication paths. Software diversity could involve independent calculations of the process state using different types of information-temperature and pressure calculations in one piece of software compared to pressure and neutron flux in another pieceeither of which is sufficient to determine, in time, if backup systems must be started to achieve safety functions.

Finally, quality parts and design are used to reduce the probability of any individual failures from occurring.

\subsection{Software Hazard Analysis as Part of Software Design}

The ultimate objectives of any hazard analysis program are to identify and correct deficiencies and to provide information on the necessary safeguards. This is certainly true of Software Hazard Analysis. There is no point to the analysis unless appropriate action is taken. At least four types of actions may be appropriate, depending on the circumstances:

1. The system design may be changed to eliminate identified hazards which are affected by software or are not adequately handled by software, or to reduce the hazards to acceptable levels, or to adjust the system architecture so that identified hazards are compensated by defense-indepth.

2. The software design may be changed to eliminate identified hazards, or reduce them to acceptable levels.

3. The quality of the software may be improved sufficiently to reduce the probability of a hazard to an acceptable level.

4. The application system may be rejected if it is considered too hazardous.

\subsection{General Approach to Software Hazard Analysis}

Software hazard analysis should be a defined aspect of the software life cycle. No specific life 
cycle is endorsed here (see Lawrence (1993) for a discussion of life cycles). To provide some specificity to the discussion, a waterfall life cycle is assumed, as shown in Figure 1. Not all the phases in the figure are included in the hazard analysis.

Hazard analysis begins with analyses of the reactor design which identify parameter limits of the safe operating region for the thermalhydraulic properties of the reactor. This provides a variety of documents which serve as the starting point for the software hazard analysis. The general approach is shown in Figure 2, which shows the technical development activities (requirements, architecture, design, code), the V\&V activities, and the hazard analysis activities. Results of the various software hazard analyses are used, as appropriate, to change the protection system design, change the software architecture or design, and to identify portions of the software which require increased attention to quality.

This report does not discuss methods or techniques for performing the recommended hazard analyses. Little extensive experience with analysis techniques has been reported in the literature. Hazard and Operability Analysis (HAZOP), Failure Modes and Effects Analysis (FMEA) and Fault Tree Analysis (FTA) are possibilities (see Appendix A). Other potential possibilities are listed in Appendix B.

\subsection{Prerequisites to Software Hazard Analysis}

Considerable work is required before a software hazard analysis process can begin. The following list will generally require some modifications to fit specific projects. Since iterations of analyses are necessary as the software development proceeds, no strict chronological rigidity is implied. For example, a Preliminary Hazard Analysis is needed before a Software Requirements Hazard Analysis can take place. However, the results of that analysis or some other requirements analysis might result in a system design change, which in turn might require modifications to the Preliminary Hazard Analysis.
Each of the prerequisite steps should result in one or more documents. These will be required in order to perform the various software hazard analyses.

1. Prepare a Preliminary Hazard List (PHL) for the application system. This will contain a list of all identified hazards, and will generally be based on the reactor Safety Analysis Report and the list of Postulated Initiating Events (PIE).

2. Prepare a Preliminary Hazard Analysis (PHA) for the application system and subsystems which have impact on the software. This evaluates each of the hazards contained in the PHL, and should describe the expected impact of the software on each hazard.

It is recommended that the PHA assign a preliminary severity level to each hazard. The method outlined in IEC 1226 is acceptable (see Appendix A.1.4 for a discussion). This method assigns a level code of $\mathrm{A}, \mathrm{B}$ or $\mathrm{C}$ to each hazard, where " $\mathrm{A}$ " is assigned to the most critical software.

3. Carry out the required hazard investigations and evaluations at the application system and application subsystem level. This should include an evaluation of the impact of software on hazards.

There are at least four potential impacts of software on each hazard (see IEEE 1228, discussed in Appendix A.1.1). These are:

a. The software may challenge the reactor safety systems; failure of the software to operate correctly has the potential for creating a hazardous condition that must be removed or mitigated by some other system. An example is a software-based reactor control system whose failure may initiate a reactor transient that causes reactor operation to diverge toward an unsafe operating region. 
Section 2. Introduction to the Software Hazard Analysis Process

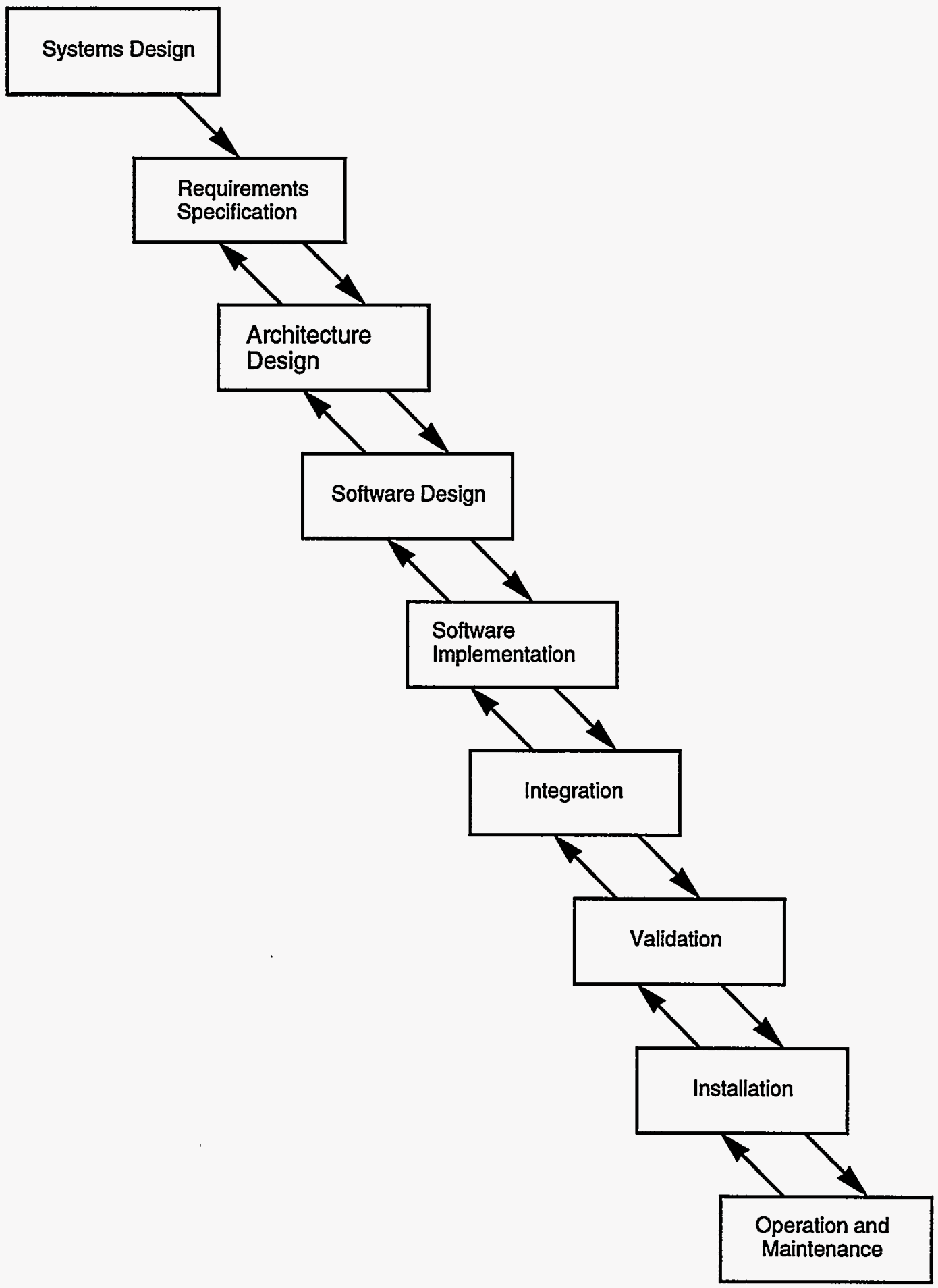

Figure 1. Waterfall Life Cycle Model 


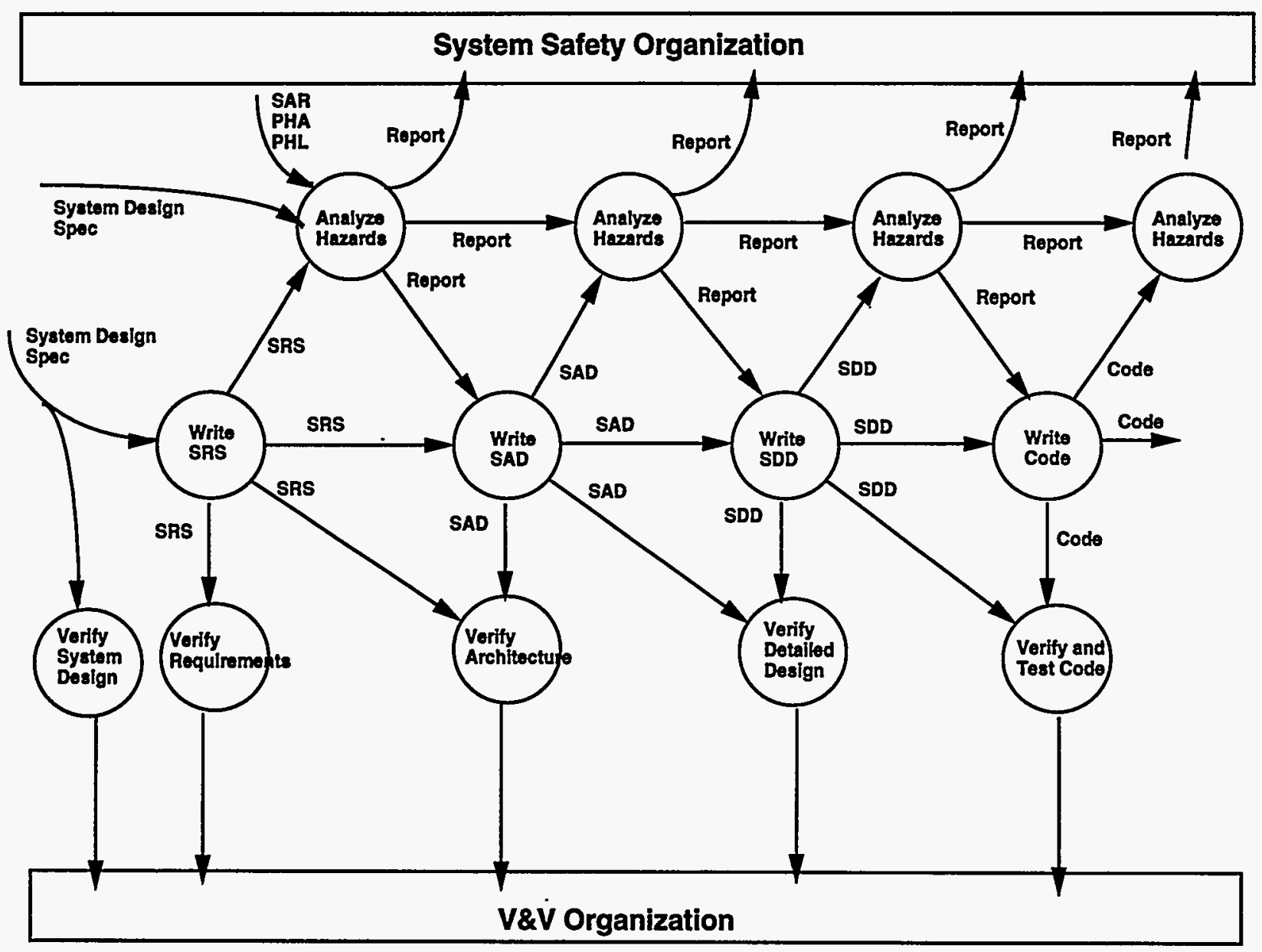

Figure 2. Software Hazard Analysis within the Software Life Cycle

\section{Abbreviations}

PHA Preliminary Hazard Analysis

PHL Preliminary Hazard Analysis

SAD Software Architecture Description

SAR Safety Analysis Report

SDD Software Design Description

SRS Software Requirements Specification 
b. The software may be responsible for preventing a hazard from progressing to an incident; failure of the software to operate correctly has the potential for converting the hazard to an accident. An example is software control of the. reactor trip system, where potential failure of this system during an emergency would permit a reactor transient to progress to a significant event.

c. The software may be used to move the system from a hazardous state to a nonhazardous state, where the hazardous state is caused by some portion of the application system other than the software. Software controlling the emergency core cooling systems is an example of this, where decay heat is removed to move a reactor from hot to cold shutdown when other cooling systems are unavailable.

d. The software may be used to mitigate the consequences of an accident. An example is software controlling the containment isolation system, which prevents a radiation release inside the containment structure from escaping and affecting the general public.

4. Assign a consequence level and probability of occurrence to each identified hazard. The tables shown in Figures 3 and 4 can be used as a basis for this. These tables are based on IEC 1226 and MilStd 882C, and are discussed in Appendix A.1.4 and A.1.2, respectively.

5. Prepare a table like that in Figure 5 from the tables created in step 4. This table can be used to derive an estimate of risk for each hazard.

This table matches the hazard severity categories of Figure 3 to the hazard probability levels of Figure 4 to obtain a measure of overall risk. Thus, events with critical severity and occasional probability of occurrence are judged to have high risk.
6. For each hazard identified in the PHL, PHA or other hazard analyses, identify its risk level using the table prepared in step 5 .

7. Prepare an application system requirements specification.

8. Create and document a system design, which shows the allocation of safety functions to software components and other system components and shows how the software component and the remaining application system components will coordinate to address the hazards discovered in previous analyses.

9. Prepare the remaining documents to the extent required in order to specify, design, implement, verify and analyze the software component of the RPS. This includes analysis of additional hazards introduced by choice of specific digital hardware, computer language, compiler, software architecture, software design techniques, and design rules. This analysis will be revisited as digital system design and software design are elaborated.

\section{REQUIREMENTS HAZARD ANALYSIS}

Software requirements hazard analysis investigates the impact of the software requirements specification on system hazards. Requirements can generally be divided into sets, each of which addresses some aspect of the software. These sets are termed qualities here. A recommended list of qualities to be considered during software hazard analysis is given in Figure 6. Some variations may be required to match special situations.

The general intent of software requirements hazard analysis is to examine each quality, and each requirement within the quality, to assess the likely impact on hazards. McDermid et al. $(1994,1995)$ suggest the use of guide words to assess impact; this idea is adapted here. A set of guide phrases is supplied for each quality that can be used to help assess the impact on hazards of each requirement associated with the quality. 
These guide phrases are shown in Figure 7. This figure suggests concepts to be examined for each requirement that relates to specific software qualities. In some cases, a requirement may affect more than one quality. The figure lists the various qualities; in some cases, these are further divided into aspects of the quality. The third column contains a code for the life cycle phase during which use of the guide phrase is recommended.
Letters are:

$\mathrm{R}$ Requirements

A Architectural Design

D Detailed Design

C Coding

The last column contains the guide phrases. In addition to the phrases listed, the analyst should examine the impact on hazards if the requirement is actually met.

\begin{tabular}{|l|c|l|}
\hline \multicolumn{1}{|c|}{ Description } & Category & \multicolumn{1}{c|}{ Definition } \\
\hline \hline Catastrophic & A & Death, system loss, or severe environmental damage \\
\hline Critical & B & $\begin{array}{l}\text { Severe injury, severe occupational illness, major } \\
\text { system or environmental damage }\end{array}$ \\
\hline Marginal & C & $\begin{array}{l}\text { Minor injury, minor occupational illness or minor } \\
\text { system or environmental damage }\end{array}$ \\
\hline Negligible & -- & $\begin{array}{l}\text { Less than minor injury, occupational illness or less } \\
\text { than minor system or environmental damage }\end{array}$ \\
\hline
\end{tabular}

Figure 3. Hazard Severity Categories (based on IEC 1126)

\begin{tabular}{|l|c|l|}
\hline \multicolumn{1}{|c|}{ Description } & Level & \multicolumn{1}{|c|}{ Estimate of Probability } \\
\hline \hline Frequent & A & Likely to occur frequently \\
\hline Probable & B & $\begin{array}{l}\text { Will occur several times in the } \\
\text { life of an item }\end{array}$ \\
\hline Occasional & C & $\begin{array}{l}\text { Likely to occur some time in the } \\
\text { life of an item }\end{array}$ \\
\hline Remote & D & $\begin{array}{l}\text { Unlikely but possible to occur } \\
\text { in the life of an item }\end{array}$ \\
\hline Improbable & E & $\begin{array}{l}\text { So unlikely, it can be assumed } \\
\text { occurrence may not be } \\
\text { experienced }\end{array}$ \\
\hline
\end{tabular}

Figure 4. Hazard Probability Levels (based on Mil-Std 882C) 


\begin{tabular}{|l||c|c|c|c|}
\hline \multicolumn{1}{|c||}{ Frequency } & \multicolumn{4}{c|}{ Hazard Category } \\
\cline { 2 - 5 } & Catastrophic & Critical & Marginal & Negligible \\
\hline \hline Frequent & High & High & High & Medium \\
\hline Probable & High & High & Medium & Low \\
\hline Occasional & High & High & Medium & Low \\
\hline Remote & High & Medium & Low & Low \\
\hline Improbable & Medium & Low & Low & Low \\
\hline
\end{tabular}

Figure 5. Example Matrix for Determining Risk

\begin{tabular}{|c|c|}
\hline Quality & Description of Quality \\
\hline Accuracy & $\begin{array}{l}\text { The term accuracy denotes the degree of freedom from error of sensor and } \\
\text { operator input, the degree of exactness possessed by an approximation or } \\
\text { measurement, and the degree of freedom of actuator output from error. }\end{array}$ \\
\hline Capacity & $\begin{array}{l}\text { The terms capacity denotes the ability of the software system to achieve its } \\
\text { objectives within the hardware constraints imposed by the computing } \\
\text { system being used. The main factors of capacity are Execution Capacity } \\
\text { (timing) and Storage Capacity (sizing). These refer, respectively, to the } \\
\text { availability of sufficient processing time and memory resources to satisfy } \\
\text { the software requirements. }\end{array}$ \\
\hline Functionality & $\begin{array}{l}\text { The term functionality denotes the operations which must be carried out by } \\
\text { the software. Functions generally transform input information into output } \\
\text { information in order to affect the reactor operation. Inputs may be obtained } \\
\text { from sensors, operators, other equipment or other software as appropriate. } \\
\text { Outputs may be directed to actuators, operators, other equipment or other } \\
\text { software as appropriate. }\end{array}$ \\
\hline Reliability & $\begin{array}{l}\text { The term reliability denotes the degree to which a software system or } \\
\text { component operates without failure. This definition does not consider the } \\
\text { consequences of failure, only the existence of failure. Reliability } \\
\text { requirements may be derived from the general system reliability } \\
\text { requirements by imposing reliability requirements on the software } \\
\text { components of the application system which are sufficient to meet the } \\
\text { overall system reliability requirements. }\end{array}$ \\
\hline Robustness & $\begin{array}{l}\text { The term robustness denotes the ability of a software system or component } \\
\text { to function correctly in the presence of invalid inputs or stressful } \\
\text { environmental conditions. This includes the ability to function correctly } \\
\text { despite some violation of the assumptions in its specification. }\end{array}$ \\
\hline Safety & $\begin{array}{l}\text { The term safety is used here to denote those properties and characteristics } \\
\text { of the software system that directly affect or interact with system safety } \\
\text { considerations. The other qualities discussed in this table are important } \\
\text { contributors to the overall safety of the software-controlled protection } \\
\text { system, but are primarily concerned with the internal operation of the } \\
\text { software. This quality is primarily concerned with the affect of the software } \\
\text { on system hazards and the measures taken to control those hazards. }\end{array}$ \\
\hline Security & $\begin{array}{l}\text { The term security denotes the ability to prevent unauthorized, undesired } \\
\text { and unsafe intrusions. Security is a safety concern in so far as such } \\
\text { intrusions can affect the safety-related functions of the software. }\end{array}$ \\
\hline
\end{tabular}

Figure 6. Software Qualities Relating to Potential Hazards 


\begin{tabular}{|c|c|c|c|}
\hline Quality & Aspect & Phase & Guide Phrases \\
\hline \multirow[t]{27}{*}{ Accuracy } & \multirow[t]{8}{*}{ Sensor } & RADC & Stuck at all zeroes \\
\hline & & RADC & Stuck at all ones \\
\hline & & RADC & Stuck elsewhere \\
\hline & & RADC & Below minimum range \\
\hline & & RADC & Above maximum range \\
\hline & & RADC & Within range, but wrong \\
\hline & & RADC & Physical units are incorrect \\
\hline & & RADC & Wrong data type or data size \\
\hline & \multirow[t]{8}{*}{ Actuator } & RADC & Stuck at all zeroes \\
\hline & & RADC & Stuck at all ones \\
\hline & & RADC & Stuck elsewhere \\
\hline & & RADC & Below minimum range \\
\hline & & RADC & Above maximum range \\
\hline & & RADC & Within range, but wrong \\
\hline & & RADC & Physical units are incorrect \\
\hline & & RADC & Wrong data type or data size \\
\hline & \multirow[t]{6}{*}{$\begin{array}{l}\text { Operator Input \& } \\
\text { Output }\end{array}$} & RA & Numerical value below acceptable range \\
\hline & & RA & Numerical value above acceptable range \\
\hline & & $\mathrm{RA}$ & Numerical value within range, but wrong \\
\hline & & RA & Numerical value has wrong physical units \\
\hline & & RA & $\begin{array}{l}\text { Numerical value has wrong data type or data } \\
\text { size }\end{array}$ \\
\hline & & $\mathrm{RA}$ & Non-numerical value incorrect \\
\hline & \multirow[t]{5}{*}{ Calculation } & RDC & $\begin{array}{l}\text { Calculated result is outside acceptable error } \\
\text { bounds (too low) }\end{array}$ \\
\hline & & RDC & $\begin{array}{l}\text { Calculated result is outside acceptable error } \\
\text { bounds (too high) }\end{array}$ \\
\hline & & $\mathrm{RDC}$ & Formula or equation is wrong \\
\hline & & RDC & Physical units are incorrect \\
\hline & & $\mathrm{RDC}$ & Wrong data type or data size \\
\hline
\end{tabular}

Figure 7. Guide Phrases for Software Qualities 


\begin{tabular}{|c|c|c|c|}
\hline \multirow[t]{23}{*}{ Capacity } & \multirow[t]{8}{*}{ Message } & RADC & Message volume is below stated minimum \\
\hline & & RADC & Message volume exceeds stated maximum \\
\hline & & RADC & Message volume is erratic \\
\hline & & RADC & Message rate is below stated minimum \\
\hline & & RADC & Message rate exceeds stated maximum \\
\hline & & RADC & Message rate is erratic \\
\hline & & RADC & Message contents are incorrect, but plausible \\
\hline & & RADC & Message contents are obviously scrambled \\
\hline & \multirow[t]{15}{*}{ Timing } & RADC & Input signal fails to arrive \\
\hline & & RADC & Input signal occurs too soon \\
\hline & & RADC & Input signal occurs too late \\
\hline & & RADC & Input signal occurs unexpectedly \\
\hline & & RADC & System behavior is not deterministic \\
\hline & & RADC & Output signal fails to arrive at actuator \\
\hline & & RADC & Output signal arrives too soon \\
\hline & & RADC & Output signal arrives too late \\
\hline & & RADC & Output signal arrives unexpectedly \\
\hline & & $\mathrm{R}$ & Insufficient time allowed for operator action \\
\hline & & $\mathrm{AD}$ & Processing occurs in an incorrect sequence \\
\hline & & $\overline{\mathrm{DC}}$ & Code enters non-terminating loop \\
\hline & & $\mathrm{DC}$ & Deadlock occurs \\
\hline & & $\mathrm{C}$ & Interrupt loses data \\
\hline & & $\mathrm{C}$ & Internupt loses control information \\
\hline \multirow[t]{10}{*}{ Functionality } & & RA & $\begin{array}{l}\text { Function is not carried out as specified (for } \\
\text { each mode of operation) }\end{array}$ \\
\hline & & $\mathrm{RA}$ & $\begin{array}{l}\text { Function is not initialized properly before } \\
\text { being executed }\end{array}$ \\
\hline & & $\mathrm{RA}$ & $\begin{array}{l}\text { Function executes when trigger conditions } \\
\text { are not satisfied }\end{array}$ \\
\hline & & $\mathrm{RA}$ & $\begin{array}{l}\text { Trigger conditions are satisfied but function } \\
\text { fails to execute }\end{array}$ \\
\hline & & RA & $\begin{array}{l}\text { Function continues to execute after } \\
\text { termination conditions are satisfied }\end{array}$ \\
\hline & & $\overline{\mathrm{RA}}$ & $\begin{array}{l}\text { Termination conditions are not satisfied but } \\
\text { function terminates }\end{array}$ \\
\hline & & $\mathrm{RA}$ & $\begin{array}{l}\text { Function terminates before necessary actions, } \\
\text { calculations, events, etc. are completed }\end{array}$ \\
\hline & & $\mathrm{R}$ & $\begin{array}{l}\text { Function is executed in incorrect operating } \\
\text { mode }\end{array}$ \\
\hline & & $\mathrm{R}$ & Function uses incorrect inputs \\
\hline & & $\mathrm{R}$ & Function produces incorrect outputs \\
\hline
\end{tabular}

Figure 7. Guide Phrases for Software Qualities, continued 


\begin{tabular}{|c|c|c|}
\hline \multirow[t]{14}{*}{ Reliability } & RA & Software is less reliable than required \\
\hline & $\overline{\mathrm{RA}}$ & Software is more reliable than required \\
\hline & $\overline{\mathrm{RA}}$ & $\begin{array}{l}\text { Software reliability is not known when the } \\
\text { system goes into production use }\end{array}$ \\
\hline & RA & $\begin{array}{l}\text { Software does not degrade gracefully when } \\
\text { required (crashes instead) }\end{array}$ \\
\hline & RA & $\begin{array}{l}\text { Software fault tolerance requirements (if } \\
\text { any) are not met }\end{array}$ \\
\hline & $\overline{R A}$ & $\begin{array}{l}\text { Reliability varies among the different modes } \\
\text { of operation }\end{array}$ \\
\hline & $\mathrm{R}$ & Software fails in-service test \\
\hline & $\mathbf{R}$ & Software fails \\
\hline & $\bar{A}$ & Hardware unit fails \\
\hline & $\overline{\mathrm{A}}$ & $\begin{array}{l}\text { Software failure propagates to uninvolved } \\
\text { processes }\end{array}$ \\
\hline & $\mathrm{A}$ & Software fails to recover from failure \\
\hline & $\mathrm{A}$ & $\begin{array}{l}\text { Hardware or software failure is not reported } \\
\text { to operator }\end{array}$ \\
\hline & $\overline{\mathrm{A}}$ & $\begin{array}{l}\text { Software fails to detect inappropriate } \\
\text { operation action }\end{array}$ \\
\hline & $\mathrm{AD}$ & Data is passed to incorrect process \\
\hline \multirow[t]{6}{*}{ Robustness } & RA & $\begin{array}{l}\text { Software fails in the presence of unexpected } \\
\text { input data }\end{array}$ \\
\hline & $\mathrm{RA}$ & $\begin{array}{l}\text { Software fails in the presence of incorrect } \\
\text { input data }\end{array}$ \\
\hline & $\overline{\mathrm{RA}}$ & $\begin{array}{l}\text { Software fails when anomalous conditions } \\
\text { occur }\end{array}$ \\
\hline & RA & Software fails to recover itself when required \\
\hline & $\overline{\mathrm{RA}}$ & Software fails during message overload \\
\hline & $\mathrm{RA}$ & Software fails when messages missed \\
\hline \multirow[t]{4}{*}{ Safety } & RA & $\begin{array}{l}\text { Software causes system to move to a } \\
\text { hazardous state }\end{array}$ \\
\hline & RA & $\begin{array}{l}\text { Software fails to move system from } \\
\text { hazardous to nonhazardous state }\end{array}$ \\
\hline & $\mathrm{RA}$ & $\begin{array}{l}\text { Software fails to initiate emergency } \\
\text { shutdown when required to do so }\end{array}$ \\
\hline & $\mathrm{RA}$ & $\begin{array}{l}\text { Software fails to recognize hazardous reactor } \\
\text { state }\end{array}$ \\
\hline \multirow[t]{3}{*}{ Security } & $\overline{R A}$ & $\begin{array}{l}\text { Unauthorized person has access to software } \\
\text { system }\end{array}$ \\
\hline & $\mathrm{RA}$ & $\begin{array}{l}\text { Unauthorized changes have been made to } \\
\text { software }\end{array}$ \\
\hline & $\mathrm{RA}$ & $\begin{array}{l}\text { Unauthorized changes have been made to } \\
\text { plant data }\end{array}$ \\
\hline
\end{tabular}

Figure 7. Guide Phrases for Software Qualities, continued 
Numerous traditional qualities generally considered necessary to an adequate software requirements specification are not included in Figure 7. Completeness, consistency, correctness, traceability, unambiguity and verifiability are, of course, necessary, but should be handled as part of requirements analysis and verification, not as part of hazards analysis.

For example, the first quality is sensor accuracy. Suppose there were an accuracy requirement for a particular sensor that "The value from sensor 123 shall be between 100 and 500, with an error of no more than 5\%." Then, the following questions should be asked:

- What is the effect on hazards if the sensor reading satisfies the requirement? In particular, what if the reading is $5 \%$ away from the actual value?

- What is the effect on hazards if the sensor is stuck at all zeros?

- What if the sensor is stuck at all ones?

- What if the sensor value is less than 100 ?

- What if the sensor value is greater than 500 ?

- What if the sensor value is between 100 and 500 , but is not within $5 \%$ of the actual value?

It is important that this analysis not be sidetracked into asking how such conditions might occur, or into arguments on the impossibility of the conditions. For hazard analysis, assume that the conditions can occur, and examine the consequences.

\subsection{Inputs to Software Requirements Hazard Analysis}

The following information should be available to perform the requirements hazard analysis.

- Preliminary Hazard List

- Preliminary Hazard Analysis
- Safety Analysis Report

- Protection System Design Description

- Software Requirements Specification

\subsection{Analysis Procedures}

The following steps may be used to carry out the requirements hazard analysis. The steps are meant to help organize the process. Variations in the process, as well as overlap in time among the steps, is to be expected.

1. Identify the hazards for which software is in any way responsible. This identification includes an estimate of the risk associated with each hazard.

2. Identify the software criticality level associated with each hazard and control category, using the table in Figure 5.

3. Match each safety-critical requirement in the software requirements specification (SRS) against the system hazards and hazard categories in order to assign a criticality level to each requirement.

4. Analyze each requirement using the guide phrases in Figure 7 which are marked with an "R." These guide phrases are meant to initiate discussion and suggest possibilities to consider, not to bound the analysis.

There are a great many phrases in Figure 7. For any particular requirement, most of these will not apply. For example, only about eight of the phrases would apply to the example given at the beginning of Section 3. Part of the analysis of this step is to select the quality or qualities that apply to the requirement, so that only applicable phrases are used.

5. Document the results of the analysis. 
The information collected during this hazard analysis can be of considerable use later during software development. The combination of criticality level assigned to the various software requirements and the guide phrase analysis provides information on the assignment of resources during further development, verification and testing. It can also suggest the need for redesign of the application system to reduce software-affected hazards.

It is possible that the Software Requirements Hazard Analysis leads to the conclusion that some changes should be made to the system design. In particular, it might be discovered that some system requirements assigned to software can be better met through hardware.

It is likely that the hazard analysis will conclude that some requirements do not pose hazardsthat is, there are no circumstances where failure to satisfy the requirements can cause a hazard. Such requirements probably do not need to be considered in the following analysis steps.

There are many ways to carry out the analysis of step 4. The technique most prominently documented in the literature is Fault Tree Analysis (FTA) (see Appendix A.4 for a discussion). Event Tree Analysis (ETA) should also be considered, using the guide phrases as top events in the tree and expanding the tree to consider consequences. The choice of technique depends on what information is known to the analyst and what information is sought.

\subsection{Outputs of Software Requirements Hazard Analysis}

The products of the requirements hazard analysis consist of the following items:

- A list of software hazards.

- A criticality level for each hazard that can be affected by the software.

- A criticality level for each software requirement.

- An analysis of the impact on hazards of the software when it operates correctly or incorrectly with respect to meeting each requirement.

\section{ARCHITECTURAL DESIGN HAZARD ANALYSIS}

Software design hazard analysis is divided here into two sections: one which examines the computer system architecture, and one which examines the detailed software design. The former is discussed in this chapter.

A computer system architecture consists of three segments: the hardware architecture, the software architecture and the mapping between them. The hardware architecture describes the various hardware elements: processors, memories, disk drives, display devices and communication lines. The software architecture describes the various software processes, data stores, screen layouts and logical communication paths. The mapping describes how the software will operate on the hardware; this includes identifying which processes will operate on which processors, where the various data stores will be located, where the various screens will be displayed, and how logical communications will take place over physical paths.

Some architectures may introduce complex functions or may have failure modes that other architectures do not have. These represent additional hazards introduced by design choices and which are not identified by previous hazards analyses.

The architectural design documents should contain a two-way trace between requirements and design elements. Each requirement is traced to the design elements that implement that requirement, and each design element is traced back to the requirements which it implements. If this trace does not exist, it should be created before the architecture hazard analysis begins.

The analysis here builds on the requirements hazard analysis by extending the latter to the software architecture. A similar analysis is recommended for the hardware architecture and 
the overall computer system architecture (hardware, software and mapping).

For example, suppose there is a timing requirement that a certain signal be sent to a particular valve actuator within five seconds of receiving an overload signal from a particular sensor. This requirement would have been analyzed as part of the software requirements hazard analysis. Now, suppose the logical data path is as shown in Figure 8. Among other guide phrases that apply, the analyst should consider the effect on the hazard if the message to be sent on path "c" never arrives. In this instance, a hazard that did not exist previously has been added by the decision to implement the logical data path "c."

\subsection{Inputs to Software Architecture Hazard Analysis}

The following information should be available to perform the architecture hazard analysis.

- Preliminary Hazard List

- Preliminary Hazard Analysis

- Safety Analysis Report

- Software Requirements Specification

- Software Requirements Hazard Analysis

- Requirements to Architecture Trace Matrix

- Software Architecture Description

\subsection{Analysis Procedures}

The following steps may be used to carry out the software architecture hazard analysis.

1. For each software architectural element, determine all the requirements affected by the element. This results from the trace matrix.

2. Assign a risk level to each software architectural element, based on the risk associated with all the requirements affected by the element. Figure 9 shows one method of doing this. The figure uses the risk levels taken from Figure 5, and considers the number of requirements of various risk levels affected by the element in order to assign a risk to the element. The suggested algorithm is as follows:

a. Pick one requirement. Assign the architectural element severity level to be the same as that of the requirement. If the requirement has medium severity, for example, then the initial element level is also "medium."

b. For each additional requirement, accumulate an architectural element severity estimate by estimating the severity of consequences should all of the identified requirements fail to be met simultaneously.

c. Continue until all requirements affected by the architectural element have been considered. The final architectural element risk level is the design failure probability of the architectural element times the accumulated severity associated with failure.

3. Analyze each safety-critical architectural element using the guide phrases in Figure 7 marked "A." These phrases are meant to initiate discussion and suggest possibilities to consider, not to bound the analysis.

As with the requirements discussion in Section 4.2 , there are a great many phrases in Figure 7 marked "A." For any particular architectural element, many of these will not apply. Part of the analysis of this step is to select the quality or qualities that apply to the architectural element, so that only applicable phrases are used.

4. Document the results of the analysis. 


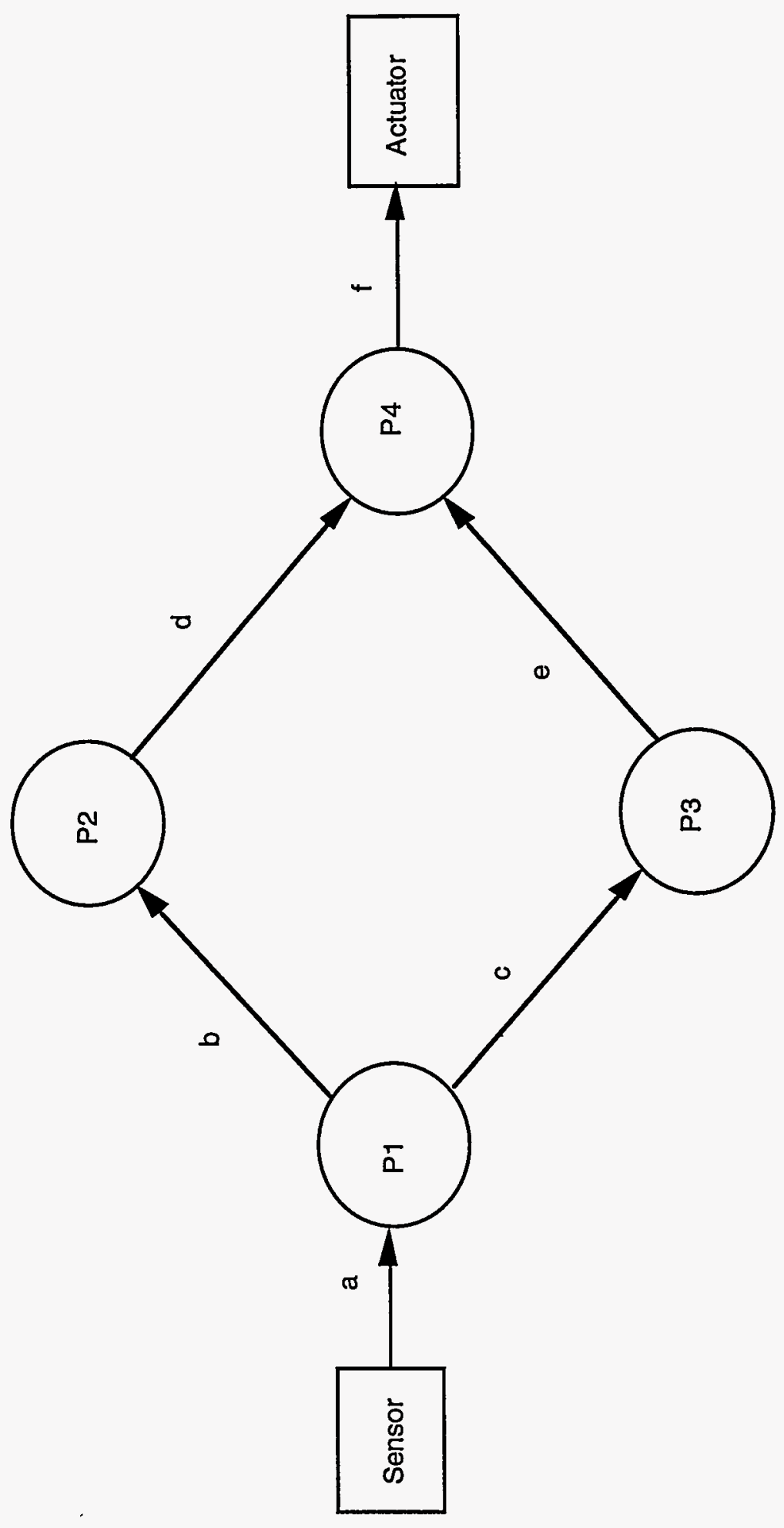

Figure 8. Example of a Software Architecture 


\begin{tabular}{|l||l|l|l|}
\hline \multirow{2}{*}{$\begin{array}{l}\text { Architecture Element } \\
\text { Risk } \\
\text { Level }\end{array}$} & \multicolumn{3}{|c|}{ Risk Level from Adding a Requirement } \\
\cline { 2 - 4 } & High & Medium & Low \\
\hline \hline Very High & Very High & Very High & Very High \\
\hline High & Very High & High & High \\
\hline Medium & High & Medium & Medium \\
\hline Low & High & Medium & Low \\
\hline
\end{tabular}

Figure 9. Determination of Architecture Risk Levels

The information collected during this analysis can supplement that of the software requirements hazard analysis. In particular, if several architectural elements are classified as very-high-risk, consideration should be given to redesigning the architecture, either to lower the risk associated with the software architecture or to provide compensatory mechanisms to lower overall application system risk. As with the requirements hazard analysis, assignment of resources to further development, verification, and testing can be based on this hazard analysis.

Architecture hazard analysis is likely to demonstrate that some architectural elements are nonhazardous; that is, the analysis shows that no possible failure of the element can affect a system hazard. Such elements require only minimal attention during design and implementation hazard analysis.

If FTA or ETA were used during the requirements hazard analysis, they may be extended to include the software and hardware architectures. The value of the trees comes mostly in the information contained in the structure of the trees. It is not likely to be possible to make a convincing assignment of failure probabilities to architectural elements, so using the tree to attempt to calculate the probability of root events should be used as a reality check and resource allocation tool only.

\subsection{Outputs of Software Architecture Hazard Analysis}

The products of the architecture hazard analysis consist of the following items:

- A list of software architectural design elements with assigned risk level.
- Analysis of the impact on hazards of the software when the specified architecture is used.

- A list of design constraints and coding constraints which are required to mitigate hazards associated with the chosen architecture.

- Recommendations for design changes which will reduce the hazard criticality level of software elements.

- Recommendations for increased analysis and testing to be carried out during detailed design V\&V, code $V \& V$ and final system validation analysis and testing.

\section{DETAILED DESIGN HAZARD ANALYSIS}

The detailed design documents should contain a two-way trace among the software requirements, the software architecture and the detailed design. Each requirement is traced through the architecture to the detailed design elements that implement the requirement. Each detailed design element is traced back through the architecture to the requirements which it implements. If this trace does not exist, it should be created before this hazard analysis begins.

The primary task here is to see if the detailed design changes any of the results of the requirements or architecture hazard analyses. If the latter have been performed carefully and completely, there should be little more to do. Verification becomes of increasing importance at this point in the life cycle, using the results of the hazard analyses to direct the verification activities. 


\subsection{Inputs to Software Detailed Design Hazard Analysis}

The following information should be available to perform the architecture hazard analysis.

- Preliminary Hazard List

- Preliminary Hazard Analysis

- Safety Analysis Report

- Software Requirements Specification

- Software Architecture Description

- Software Detailed Design Description

- Software Requirements and Architecture

- Hazard Analyses

- Trace Matrix, Requirements to Architecture to Detailed Design

\subsection{Analysis Procedures}

The following steps may be used to carry out the software detailed design hazard analysis.

1. For each software architecture element, prepare a list of detailed design elements which together constitute the architectural element. It may happen that some design elements are used in more than one architectural element. For example, low level communication software may be used by almost every element of the architecture. Device drivers are additional examples.

2. For each design element, use the guide phrases in Figure 7 that are marked " $D$ " to determine if the hazards associated with the architecture elements have changed. This may occur if design elements, design rules, design tools, or design techniques introduce common-mode failure mechanisms to two or more architectural elements. If so, previous hazard analyses may need to be redone.

3. Document the results.

If resources do not exist to analyze all design elements, choose those elements that (1) constitute architectural elements of very high or high risk and (2) those elements that occur in many architectural elements. The latter are most likely service elements, such as communications modules, device drivers or file managers.

It should be expected that, in most cases, the analysis will quickly determine that there has been no change to systems hazards due to the detailed design. That is, if a careful job has been done in identifying, controlling and mitigating hazards during the requirements and architecture phrases, there should be little left to do at the detailed design phase. If this is true, emphasis can start shifting from the global concern of systems hazards to the more local concern of implementation correctness.

The information collected during this analysis can help provide assurance that no new hazards have been introduced by the detailed design. It can also help with the assignment of resources for coding and testing.

\subsection{Outputs of Software Detailed Design Hazard Analysis}

The product of the software detailed design hazard analysis consists of the documented analysis.

\section{CODE HAZARD ANALYSIS}

The software documents should contain a twoway trace between the detailed design element and the code elements which implement the design elements. If this trace does not exist, it should be created before code hazard analysis begins.

Correctness is much more a concern at this point than hazard analysis, provided that the previous three analyses have been performed well. The main emphasis is on making sure that nothing in the code changes the previous analyses or creates a new hazard. Results of the previous analyses can be used to direct verification and testing resources to the most critical code elements. 


\subsection{Inputs to Software Code Hazard Analysis}

The following information should be available to perform the architecture hazard analysis.

- Preliminary Hazard List

- Preliminary Hazard Analysis

- Safety Analysis Report

- Software Requirements Specification

- Software Architecture Description

- Software Detailed Design Description

- Code

- Software Requirements, Architecture and Design Hazard Analyses

- Trace Matrix, Requirements for Architecture to Design to Code Elements

\subsection{Analysis Procedures}

The following steps may be used to carry out the code hazard analysis.

1. For each code element, use the guide phrases in Figure 7 that are marked " $C$ " to determine if the results of the design hazard analysis need to be modified or if new hazards have been introduced. If so, some or all of the previous analyses may need to be redone.

Resources are not likely to exist to analyze all code elements. Concentrate on those that encode the most risky design elements and those that support basic computing system functions.

2. Examine tools, computer language, and coding techniques for their potential to introduce common-mode failure mechanisms to all modules. Identify coding rules or tool-usage rules that avoid risky tool features or coding techniques. If a preexisting operating system will be used, identify the risky features or functions that should be avoided.

3. Document the results.

\subsection{Outputs of Software Code Hazard Analysis}

The product of the code hazard analysis consists of the documented analysis.

\section{SUMMARY AND CONCLUSIONS}

The software hazard analysis described in Sections 3-6 could require a significant effort when applied to the digital computer-based I\&C system for modern reactor control and protection systems or another process I\&C system whose failure could result in significant adverse public, environmental, or financial consequences. It must be recognized that in reality, software hazards analysis is only one of several activities necessary for the development of software to be used in safety-critical applications. Principal activities in this regard include configuration management, verification and validation, and quality assurance activities. A detailed discussion of the life cycle activities for the development of safety-critical software is given in Lawrence (1993). A summary of design factors important to the realization of highquality software that is "fit for duty" in safetycritical applications is given in Lawrence (1994).

With the above view in mind, one can then consider where software hazards analysis offers a unique capability to improve the integrity of safety-critical software. Section 2 provides an overview of the objectives of the hazards analysis activities for both system hazards and software hazards and the relation between hazards analysis activities and other principal software development life cycle activities. A major impact of the results from the software hazards analysis is on changes to the software requirements specification for the purpose of eliminating identified hazards that are affected by the software or that are not adequately managed by the software. Another major impact of these results is on the software architecture, in particular the addition of software architectural features that improve the management of hazards through the concept of defense-in-depth. 
The impact of software hazards analysis on the software design specification, with the exception of the use of potentially complex operations associated with data flow and control flow, is overshadowed by the need to address concerns related to correctness through the traceability and V\&V aspects discussed in Section 5. The emphasis on correctness is even more true for the software code. The discussion in Section 6 provides guidance on matters that are more effectively dealt with through correctness concerns.

The more detailed presentation of the software hazards analysis in Section 3, Requirements Hazards Analysis, includes an approach to guide the assessment of the impact on hazards of each requirement as it is related to the qualities given in Figure 6. The guide phrases for this assessment are presented in Figure 7.

The selection of applicable guide phrases to particular requirements must be governed by the potential impact of each software hazard on the system, as presented in item 3 of section 2.4 and the accompanying risk associated with that hazard, as given in Figure 5. Similar selection considerations are applicable for the architectural design hazards analysis described in Section 4.

In conclusion, limiting the bulk of the software hazards investigation to the software requirements specification and the software architectural design and the judicious selection of the events to be assessed should lead to a hazards analysis result that (1) minimizes the probability of occurrence of those hazards with the more significant consequences and (2) minimizes the increase in design requirements that could have the potential for an increase in the complexity of the design.

The process outlined in Chapters 3-6 is based on the documents listed in the References. It has not been tested or evaluated in the field. 



\section{REFERENCES}

Air Force Inspection and Safety Center. 1985. Software System Safety. Headquarters Air Force Inspection and Safety Center, AFISC SSH 1-1, September 5.

Bowman, William C., Glenn H. Archinoff, Vijay M. Raina, David R. Tremaine and Nancy G. Leveson. 1991. "An Application of Fault Tree Analysis to Safety Critical Software at Ontario Hydro." Probabilistic Safety Assessment and Management, G. Apostolakis, ed. (1991): 363368.

Brown, Michael L. 1985. "Software Safety for Complex Systems. IEEE Annual Conf. of the Eng. in Medicine and Biology Society (1985): 210-216.

Center for Chemical Process Safety. 1992. 1992. Guidelines for Hazard Evaluation Procedures, American Institute of Chemical.Engineers.

Clarke, Stephen J. and John A. McDermid. 1993. "Software Fault Trees and Weakest Preconditions: a Comparison and Analysis." Soft. Eng. J. 8, no. 4 (July): 225-236.

Connolly, Brian. 1989. "Software Safety Goal Verification Using Fault Tree Techniques: A Critically Ill Patient Monitor Example." Computer Assurance Conf. (Compass) (June): 18-21.

Elahi, B. John. 1993. "Safety and Hazard Analysis for Software Controlled Medical Devices." Sixth Annual IEEE Symp. On Comp.Based Med. Syst (June): 10-15.

Froome, P. K. D. 1992. Interim Def Stan 00-56: Hazard Analysis and Safety Classification of the Computer and Programmable Electronic System Elements of Defense Equipment," Centre for Software Reliability, Ninth Annual Conf. on Soft. Safety, Luxembourg (April): 1-14.

Gowen, Lon D., James S. Collofello and Frank W. Calliss. 1992. "Preliminary Hazard Analysis for Safety-Critical Software Systems," Phoenix Conf. Comp. and Comm. (April): 501-508.
Hammer, Willie. 1972. Handbook of System and Product Safety. Prentice-Hall.

IEC 1226. 1993. Nuclear Power PlantsInstrumentation and Control Systems Important for Safety-Classification. International Electrotechnical Commission.

IEEE 7-4.3.2. 1993. IEEE Standard Criteria for Digital Computers in Safety Systems of Nuclear Power Generating Stations. Institute of Electronic and Electrical Engineers.

IEEE 1228. 1994. IEEE Standard for Software Safety Plans. Institute of Electronic and Electrical Engineers.

Lal-Gabe, Anshoo. 1990. "Hazards Analysis and its Application to Build Confidence in Software Test Results." Third Annual IEEE Symp. On Comp.-Based Med. Syst. (June): 129-134.

Lawrence, J. Dennis. 1993. Software Reliability and Safety in Nuclear Reactor Protection Systems. NUREG/CR-6101, UCRL-ID-114839, Lawrence Livermore National Laboratory, November.

Lawrence, J. Dennis and G. Gary Preckshot. 1994. Design Factors for Safety-Critical Software, NUREG/CR-6294, Lawrence Livermore National Laboratory, December.

Levan, David G. 1992. "Preliminary Procedure for Software Hazard Analysis of Safety-Critical Software." Prepared for Ontario Hydro Nuclear Safety Department by DLSF Systems, Inc., January.

Leveson, Nancy G. and Peter R. Harvey. 1983. "Analyzing Software Safety." IEEE Trans. Soft. Eng. 9, no. 5 (September): 569-579.

Leveson, Nancy G. 1991a. "Safety Assessment and Management Applied to Software."

Probabilistic Safety Assessment and Management, G. Apostolakis, ed. 377-382.

Leveson, Nancy G. 1991b. "Safety." Aerospace Software Engineering. Christine Anderson and Merlin Dorfman, ed., AIAA, 319-336. 
Leveson, Nancy G., Stephen S. Cha and Timothy J. Shimeall, 1991c. "Safety Verification of Ada Programs Using Software Fault Trees." IEEE Software (July): 48-59.

Levinson, Stanley H. and H. Tazewell Doughtrey. 1993. "Risk Analysis of SoftwareDependent Systems." Prob. Safety Assessment Int'l. Topical Meeting (January).

McDermid, J. A., and D. J. Pumfrey. 1994."A Development of Hazard Analysis to Aid Software Design." Computer Assurance. (Compass) (June): 17-25.

McDermid, John A., M. Nicholson, D. J. . Pumfrey and P. Fenelon. 1995. "Experience with the Application of HAZOP to Computer-Based Systems." Computer Assurance (Compass) (June): 37-48

McKinlay, Archibald. 1991. "The State of Software Safety Engineering." Probabilistic Safety Assessment and Management, G. Apostolakis, ed. (1991): 369-376.

Mil-Hdbk-764. 1990. System Safety Engineering Design Guide for Army Materiel. U.S. Dept. of Defense (January 12).
Mil-Std 882C. 1993. System Safety Program Requirements. U.S. Dept. of Defense (January).

MOD 00-56. 1991. Hazard Analysis and Safety Classification of the Computer and Programmable Electronic System Elements of Defense Equipment. Defence Standard 00-56, Ministry of Defence, Great Britain (April).

Mojdehbakhsh, Ramin, Satish Subramanian, Ramakrishna Vishnuvajjala, Wei-Tek Tsai and Lynn Elliott. 1994. "A Process for Software Requirements Safety Analysis.” Int'l Symp. on Software Reliability Engineering, (November): 45-54.

NIST 1993. Review of Software Hazard Analyses. National Institutes of Standards and Technology. Draft (June 4).

Stephans, Richard A. and Warner W. Talso. 1993. System Safety Analysis Handbook. System Safety Society, New Mexico Chapter, Albuquerque, NM, July. 


\section{APPENDIX A. BACKGROUND}

\section{A.1. Standards Review}

\section{A.1.1. IEEE 1228, Standard for Software Safety Plans}

IEEE 1228 Standard, Standard for Software

Safety Plans, "describes the minimum acceptable requirements for the content of a Software Safety Plan." A Software Safety Plan developed to satisfy this Standard will contain information on both management and technical aspects of the development activity. The recommended contents of a Software Safety Plan, as given by the Standard, are shown in Figure 10.

Only the analyses which are required in Sections 4.2-4.4 of the Safety Plan (sections 4.4.2-4.4.4 of the Standard) are relevant to the scope of this report. The Standard itself does not require any particular types of analyses. It does contain an appendix which lists some suggested analyses.

The Standard distinguishes between the Application System and the Software System. In the context of reactors, for example, the application system might be the entire reactor, or the entire reactor protection system, and the software system is the software which is contained in the reactor control system or reactor protection system, respectively. The word "system," used here without modification, will always refer to the entire application system.

The Standard assumes that certain information will be available prior to performing any safety analyses. This information is listed next.

1. A Preliminary Hazard Analysis (PHA) and any other hazard analyses which have been performed on the entire application system or any portion of it should be available. These analyses must include the following information:

a. Hazardous application system states.

b. Sequences of actions that can cause the application system to enter a hazardous state. c. Sequences of actions intended to return the application system from a hazardous state to a nonhazardous state.

d. Actions intended to mitigate the consequences of an accident.

2. A high-level application system design should exist and specify:

a. The functions which will be performed by the software contained in the application system.

b. Any actions that will be required of the software in order to prevent the application system from entering a hazardous state.

c. Any actions that will be required of the software in order to move the application system from a hazardous state to a nonhazardous state.

d. Any actions that will be required of the software to mitigate the consequences of an accident.

3. The interfaces between the software and the rest of the application system should be completely defined.

4. A software safety plan (SSP) should exist. It will describe fully the means by which software safety analyses will be carried out for the application system. IEEE Standard 1228 may be used as a model for the SSP. If the developer prefers, the software safety plan may be included in the general system safety plan.

These will be referred to as the System Hazard Analyses, the System Design, the System Interface Specification and the Software Safety Plan, respectively.

The Appendix to the Standard suggests certain analyses which may be performed during software development. These are shown in Figure 11. 


\begin{tabular}{|c|c|c|}
\hline 1. & \multicolumn{2}{|c|}{ Purpose } \\
\hline 2. & \multicolumn{2}{|c|}{ Definitions, Acronyms and References } \\
\hline 3. & \multicolumn{2}{|c|}{ Software Safety Management } \\
\hline & 3.1. & Organization and Responsibilities \\
\hline & 3.2 . & Resources \\
\hline & 3.3. & Staff Qualifications and Training \\
\hline & 3.4 . & Software Life Cycle \\
\hline & 3.5 . & Documentation Requirements \\
\hline & 3.6. & Software Safety Program Records \\
\hline & 3.7. & Software Configuration Management Activities \\
\hline & 3.8. & Software Quality Assurance Activities \\
\hline & 3.9. & Software Verification and Validation Activities \\
\hline & 3.10. & Tool Support and Approval \\
\hline & 3.11 . & Previously Developed or Purchased Software \\
\hline & 3.12 . & Subcontract Management \\
\hline & 3.13. & Process Certification \\
\hline \multirow[t]{7}{*}{4.} & \multicolumn{2}{|c|}{ Software Safety Analyses } \\
\hline & 4.1. & Software Safety Analyses Preparation \\
\hline & 4.2. & Software Safety Requirements Analysis \\
\hline & 4.3. & Software Safety Design Analysis \\
\hline & 4.4. & Software Safety Code Analysis \\
\hline & 4.5. & Software Safety Test Analysis \\
\hline & 4.6. & Software Safety Change Analysis \\
\hline \multirow[t]{9}{*}{5.} & \multicolumn{2}{|c|}{ Post-Development } \\
\hline & 5.1. & Training \\
\hline & 5.2. & Deployment \\
\hline & & 5.2.1. Installation \\
\hline & & 5.2.2. Startup and Transition \\
\hline & & 5.2.3. Operations Support \\
\hline & 5.3. & Monitoring \\
\hline & 5.4. & Maintenance \\
\hline & 5.5. & Retirements and Notification \\
\hline 6. & Plan & pproval \\
\hline
\end{tabular}

Figure 10. Outline of a Software Safety Plan 


\begin{tabular}{|l|}
\hline Software Safety Requirements Analysis \\
Criticality Analysis \\
Specification Analysis \\
Timing and Sizing Analysis \\
Software Safety Design Analysis \\
Logic Analysis \\
Data Analysis \\
Interface Analysis \\
Constraint Analysis \\
Functional Analysis \\
Module Analysis \\
Revised Timing and Sizing Analysis \\
Software Safety Code Analysis \\
Logic Analysis \\
Data Analysis \\
Interface Analysis \\
Constraint Analysis \\
Programming Style Analysis \\
Non-critical Code Analysis \\
Revised Timing and Sizing Analysis \\
Software Safety Test Analysis \\
Unit Test Analysis \\
Interface Test Analysis \\
Subsystem Test Analysis \\
System-level Test Analysis \\
Stress Test Analysis \\
Regression Test Analysis \\
\end{tabular}

Figure 11. IEEE 1228 Suggested Safety Analyses

\section{A.1.2. Mil-Std 882C, System Safety Program Requirements}

This Standard applies to all military systems in which safety is a factor. The Standard is directed at DoD program managers, and is meant to assist them in overseeing contractors. The contractors are expected to carry out the safety program.

The Standard defines hazard severity categories which provide a qualitative measure of the worst credible accident. These are shown in Figure 12. A second table, reproduced here in Figure 13, categorizes the probability that a hazard will be created during the planned life expectancy of the system. This latter table is also qualitative, and is given both in terms of specific individual items, and in terms of all items in the inventory. The Standard points out that the two tables may need to be modified in some cases to fit individual situations. 
The Standard presents detailed requirements as tasks. These are organized into four sections, with specific tasks in each section. This grouping is intended to facilitate understanding, and does not imply that the tasks are to be carried out in the order listed. The task sections and individual tasks are listed in Figure 14.

It is possible to combine Figures 12 and 13 to show a hazard risk level. One way of doing this is shown in Figure 15. This latter table can be used in a hazard analysis in order to manage risk. For example, if a hazard falls in the "high" risk category, it might be possible to redesign the system or use better quality parts in order to move to a "medium" risk category. Figure 15 can also be used to determine where assessment resources should be concentrated.

\begin{tabular}{|l|c|l|}
\hline \multicolumn{1}{|c|}{ Description } & Category & \multicolumn{1}{c|}{ Definition } \\
\hline \hline Catastrophic & I & Death, system loss, or severe environmental damage \\
\hline Critical & II & $\begin{array}{l}\text { Severe injury, severe occupational illness, major } \\
\text { system or environmental damage }\end{array}$ \\
\hline Marginal & III & $\begin{array}{l}\text { Minor injury, minor occupational illness or minor } \\
\text { system or environmental damage }\end{array}$ \\
\hline Negligible & IV & $\begin{array}{l}\text { Less than minor injury, occupational illness or less } \\
\text { than minor system or environmental damage }\end{array}$ \\
\hline
\end{tabular}

Figure 12. Hazard Severity Categories (from Mil-Std 882C)

\begin{tabular}{|l|c|l|l|}
\hline \multicolumn{1}{|c|}{ Description } & Level & \multicolumn{1}{|c|}{ Specific Individual Item } & \multicolumn{1}{|c|}{ Fleet or Inventory } \\
\hline \hline Frequent & A & Likely to occur frequently & Continuously experienced \\
\hline Probable & B & $\begin{array}{l}\text { Will occur several times in the } \\
\text { life of an item }\end{array}$ & Will occur frequently \\
\hline Occasional & C & $\begin{array}{l}\text { Likely to occur some time in the } \\
\text { life of an item }\end{array}$ & Will occur several times \\
\hline Remote & D & $\begin{array}{l}\text { Unlikely but possible to occur } \\
\text { in the life of an item }\end{array}$ & $\begin{array}{l}\text { Unlikely but can reasonably be } \\
\text { expected to occur }\end{array}$ \\
\hline Improbable & E & $\begin{array}{l}\text { So unlikely, it can be assumed } \\
\text { occurrence may not be } \\
\text { experienced }\end{array}$ & Unlikely to occur, but possible \\
\hline
\end{tabular}

Figure 13. Hazard Probability Levels (from Mil-Std 882C) 


\begin{tabular}{|c|c|}
\hline Task Number & Task Title \\
\hline 100 & $\overline{\text { Program Management and Control }}$ \\
\hline 101 & System Safety Program \\
\hline 102 & System Safety Program Plan \\
\hline 103 & $\begin{array}{l}\text { Integration / Management of Associate Contractors, Subcontractors and Architect and } \\
\text { Engineering Firms }\end{array}$ \\
\hline 104 & System Safety Program Reviews and Audits \\
\hline 105 & System Safety Group / System Safety Working Group Support \\
\hline 106 & Hazard Tracking and Risk Resolution \\
\hline 107 & System Safety Progress Summary \\
\hline 200 & Design and Integration \\
\hline 201 & Preliminary Hazard List \\
\hline 202 & \begin{tabular}{|l|} 
Preliminary Hazard Analysis \\
\end{tabular} \\
\hline 203 & \begin{tabular}{|l|} 
Safety Requirements / Criteria Analysis \\
\end{tabular} \\
\hline 204 & \begin{tabular}{|l|} 
Subsystem Hazard Analysis \\
\end{tabular} \\
\hline 205 & System Hazard Analysis \\
\hline 206 & Operating and Support Hazard Analysis \\
\hline 207 & Health Hazard Assessment \\
\hline 300 & Design Evaluation \\
\hline 301 & Safety Assessment \\
\hline 302 & Test and Evaluation Safety \\
\hline 303 & $\begin{array}{l}\text { Safety Review of Engineering Change Proposals, Specification Change Notices, } \\
\text { Software Problem Reports and Requests for Deviation / Waiver }\end{array}$ \\
\hline 400 & Compliance and Verification \\
\hline 401 & Safety Verification \\
\hline 402 & Safety Compliance Assessment \\
\hline 403 & Explosive Hazard Classification and Characteristics Data \\
\hline 404 & Explosive Ordnance Disposal Data \\
\hline
\end{tabular}

Figure 14. Detailed Safety Tasks (from Mil-Std 882C)

\begin{tabular}{|l||c|c|c|c|}
\hline \multicolumn{1}{|c||}{ Frequency } & \multicolumn{4}{c|}{ Hazard Category } \\
\hline Frequent & Catastrophic & Critical & Marginal & Negligible \\
\hline Probable & High & High & High & Medium \\
\hline Occasional & High & High & Medium & Low \\
\hline Remote & High & High & Medium & Low \\
\hline Improbable & High & Medium & Low & Low \\
\hline
\end{tabular}

Figure 15. Example Matrix for Residual Risk (from Mil-Std 882C) 
An additional assessment of risk is recommended for software, which considers the potential hazard severity and the degree of control that the software exercises over the application. Four control categories are defined, as follows.

"C1. Software exercises autonomous control over potentially hazardous hardware systems $^{5}$, subsystems or components without the possibility of intervention to preclude the occurrence of a hazard. Failure of the software or a failure to prevent an event leads directly to a hazard's occurrence.

"C2a. Software exercises control over potentially hazardous hardware systems, subsystems, or components allowing time for intervention by independent safety systems to mitigate the hazard. However, these systems by themselves are not considered adequate.

"C2b. Software item displays information requiring immediate operator action to mitigate a hazard. Software failures will allow or fail to prevent the hazard's occurrence.

"C3a. Software item issues commands over potentially hazardous hardware systems, subsystems or components requiring human action to complete the control function. There are several, redundant, independent safety measures for each hazardous event.

"C3b. Software generates information of a safety critical nature used to make safety critical decisions. There are several, redundant, independent safety measures for each hazardous event.

"C4. Software does not control safety critical hardware systems, subsystems or components and does not provide safety critical information."

\footnotetext{
5 In this list, "hardware" refers to all forms of equipment, not just computer hardware. For example, a missile is considered to be hardware here.
}

From this list and the list of hazard categories, a software hazard criticality matrix can be defined. This is shown in Figure 16. Risks range from 1 to 5, which may be interpreted as follows:

1 High risk-significant analysis and testing is required.

2 Medium risk-requirements and design analysis and in-depth testing is required.

3,4 Moderate risk-high level analysis and testing is acceptable with management approval.

5 Low risk - acceptable; no additional analysis is required.

This scheme does not easily fit reactor protection systems. It addresses a primary control system which controls potentially hazardous equipment. A reactor protection system is an "independent safety system" in the sense of item C1A.

\section{A.1.3. AFISC SSH 1-1, Software System Safety Handbook}

This is a companion document to Mil-Std 882, and is designed to specifically address software. ${ }^{6}$ Software hazards fall into four broad categories:

1. Inadvertent/unauthorized event. An unexpected/unwanted event occurs.

2. Out-of-sequence event. A known and planned event occurs but not when desired.

3. Failure of event to occur. A planned event does not occur (e.g., a hazard is allowed to propagate because the program does not detect the occurrence of the hazard or fails to act).

4. Magnitude or direction of event is wrong. This is normally indicative of an algorithm error.

\footnotetext{
${ }^{6}$ Another handbook, Mil-Hdbk-764, briefly discusses software hazards analysis.
} 


\begin{tabular}{|c||c|c|c|c|}
\hline \multicolumn{1}{|c||}{} & \multicolumn{4}{c|}{ Hazard Category } \\
\hline Control Category & Catastrophic & Critical & Marginal & Negligible \\
\hline \hline C1 & 5 & 5 & 3 & 1 \\
\hline C2 & 5 & 4 & 2 & 1 \\
\hline C3 & 4 & 3 & 1 & 1 \\
\hline C4 & 3 & 2 & 1 & 1 \\
\hline
\end{tabular}

Figure 16. Example Software Hazard Criticality Matrix (from Mil-Std 882C)

The software hazard analysis effort should begin early in the software life cycle. It is intended to ensure that the software complies with safety requirements and to identify potentially hazardous conditions. Software hazard analysis (SwHA) must be fully integrated with the overall system hazard analysis. Two phases are identified: preliminary software hazard analysis (PSwHA) and follow-on software hazard analysis (FSwHA). However, it is probably better to view these as a single analysis which starts with the PSwHA and is revised as needed during software development.

The PSwHA is based on an analysis of the following documents:

1. System and subsystem PHAs

2. System and subsystem specifications

3. System allocation and interface documents

4. Functional flow diagrams and related data

5. Flow charts or their functional equivalent

6. Storage allocation and program structure documents

7. Background information related to safety requirements associated with the contemplated testing, manufacturing, storage, repair and use

8. System energy and toxic or hazardous event sources which are controlled or influenced by software

The combination of the PHA and the allocation of system functions to software can be used to identify the software components which are critical to safety. These must be investigated in depth; other components must be analyzed to ensure that their operation or failure cannot impact or influence safety-critical components.

The software hazard analyses should be revised from time to time during the development process. The handbook recommends revision after the critical design review, during coding, and during integration. Special attention should be placed on changes to requirements, design, and coding.

The handbook lists several methods of software hazard analysis; the list is not meant to be exhaustive. Software fault tree analysis, software sneak circuit analysis, nuclear safety cross-check analysis and Petri net analysis are discussed.

\section{A.1.4. IEC 1226, Classification of Safety Systems in Nuclear Power Plants}

This Standard also uses a hazard severity classification scheme, but does not weight it by probability of occurrence. Three categories are used, labeled A, B and C. The Standard is specific to nuclear reactors, so is particularly appropriate to this report. See Figure 3.

The following notations are used:

FSE Functions and the associated Systems and Equipment that implement them

I\&C Instrumentation and Control

NPP Nuclear Power Plant

PIE Postulated Initiating Event

\section{A.1.4.1. Category A}

Category A "denotes the FSE which play a principal role in the achievement or maintenance of NPP safety." An I\&C FSE falls into this category if it meets any of the following criteria: 
- It is required to mitigate the consequence of a PIE to prevent it from leading to an accident.

- Its failure when required to operate in response to a PIE could result in an accident.

- A fault or failure in the FSE would not be mitigated by another category A FSE, and would lead directly to an accident.

- It is required to provide information or control capabilities that allow specified manual actions to be taken to mitigate the consequences of a PIE to prevent it from leading to an accident.

Typical functions of a category A FSE are the following:

- Reactor shutdown and maintenance of subcriticality

- Decay heat transport to an ultimate heat sink

- Isolation of containment

- Information for essential operator action

Examples of such FSE are the reactor protection system, the safety actuation system and safety system support features. Key instrumentation and displays that permit operator actions defined in the operations manual, and required to ensure safety are also examples of category A FSEs.

\section{A.1.4.2. Category B}

Category B "denotes FSE that play a complementary role to the category A FSE in the achievement or maintenance of NPP safety." An I\&C FSE falls into this category if it meets any of the following criteria:

- It controls the plant so that process variables are maintained within safe limits.

- A requirement for operation of a category A FSE in order to avoid an accident would result from faults or failures of the category B FSE.

- It is used to prevent or mitigate a minor radioactive release, or minor degradation of fuel.
- It is provided to alert control room personnel to failures in category A FSE.

- It is provided to continuously monitor the availability of category A FSEs to accomplish their safety duties.

- It is used to reduce considerably the frequency of a PIE.

Typical functions of a category B FSE are:

- Automatic control of the reactor primary and secondary circuit conditions, keeping variables within safe limits, and prevention of events from escalating to accidents.

- Monitoring and controlling performance of individual systems and items of equipment during the post-accident phase to gain early warning of the onset of problems.

- Limiting the consequences of internal hazards.

- Monitoring or controlling the handling of fuel where a failure could cause a minor radioactive release.

Examples of category B FSE are the reactor automatic control system, control room data processing systems, fire suppression systems and safety circuits and interlocks of fuel handling systems used when the reactor is shut down.

\section{A.1.4.3. Category C}

Category $\mathrm{C}$ "denotes FSE that play an auxiliary or indirect role in the achievement or maintenance of NPP safety." An I\&C FSE falls into this category if it meets any of the following criteria:

- It is used to reduce the expected frequency of a PIE.

- It is used to reduce the demands on, or to enhance the performance of, a category A FSE.

- It is used for the surveillance or recording of conditions of FSE, to determine their safety status. 
- It is used to monitor and take mitigating action following internal hazards within the reactor design bases, such as fire and flood.

- It is used to ensure personnel safety during or following events that involve or result in release of radioactivity within the reactor, or risk radiation exposure.

- It is used to warn personnel of a significant release of radioactivity in the reactor or of a risk of radiation exposure.

- It is used to monitor and take mitigating action following natural events such as earthquakes and tornadoes.

- It is the NPP internal access control.

Typical functions of a category C FSE are:

- Those necessary to warn of internal or external hazards, such as fire, flood, explosions, earthquakes.

- Those for which operating mistakes could cause minor radioactive releases, or lead to radioactive hazard to the operators.

- Access control systems.

Examples include alarm systems, waste stream monitoring, area radiation monitoring, access control systems, and emergency communications systems.

\section{A.1.4.4. Effect of the Classification Scheme}

The primary effect is to concentrate development and assurance efforts on the most important FSEs - those of category A. An example is the use of redundancy to achieve reliability. A category A FSE is required to have redundancy so that no single point of failure exists. Redundancy is encouraged for category $B$ FSEs, but is not required if the reliability goals can be met without it. No redundancy is generally needed for category C FSEs, though it can be used if necessary to meet reliability goals.

\section{A.1.5. IEEE 7-4.3.2, Annex F, Abnormal Conditions and Events}

Annex F of IEEE 7-4.3.2 discusses the identification and resolution of abnormal conditions and events (ACEs).

ACEs are grouped into two categories, depending on their source. Some are caused by conditions or events that occur outside the computer system - a failure of a system component is an example. Others are caused by failures within the computer system.

Section F.2.3 of the Annex describes a process for identifying ACEs based on the software life cycle. It begins at the system design phase, and proceeds through computer system design, software requirements, software design, software implementation, computer integration testing and computer validation testing. The Standard lists various considerations for most of the life cycle phases; these are summarized in Fìgures 17-20.

A general procedure is provided for resolving ACEs. The intent is to eliminate ACEs or reduce the associated risk where possible. A summary of the procedure is given in Figure 21.

\section{A.2. General Discussion of Hazard Analysis}

Hammer (1972) lists six functions of hazard analysis:

1. The investigation and evaluation of the interrelationships of primary, initiating and contributory hazards that may be present.

2. The investigation and evaluation of the circumstances, conditions, equipment, personnel and other factors involved in the safety of the system and its operation.

3. The investigation and evaluation of the means of avoiding or eliminating any specific hazard by using suitable designs, procedures, processes or materials. 
4. The investigation and evaluation of the controls that may be required to prevent possible hazards and the best methods for incorporating those controls in the product or system.

5. The investigation and evaluation of the possible damaging effects resulting from lack or loss of control of any hazard that cannot be avoided or eliminated.

6. The investigation and evaluation of the safeguards for preventing injury or damage if control of the hazard is lost.

Initial hazards analyses must be carried out for the entire application system. This report assumes that the five forms of system-level hazards analyses identified in Mil-Std 882C have been carried out, except for software components. The following is a brief list of the types of hazard analysis given in the Standard:

1. Preliminary Hazard List (PHL) identifies hazards that may require safety design consideration or special analyses. It occurs upon completion of the concept definition phase of the system life cycle.

2. Preliminary Hazard Analysis (PHA) identifies and evaluates all system hazards. It starts in the concept definition phase of the system life cycle, and ends when the component-level System Hazard Analysis is able to begin. The PHA is the foundation for future system and software hazard analyses.

3. System Hazard Analysis (SHA) examines the entire system to identify hazards and assess the risk of the entire system design, including software. It starts as the system design matures, close to the design review, and is updated until the system design is complete.

4. Component SHA identifies hazards associated with the design of the components, and how those hazards will affect the entire system. It begins as each component is designed and is updated as the component design matures.

5. Operating and Support Hazard Analysis (O\&SHA) identifies and evaluates hazards related to the environment, personnel, procedures and equipment during a system operation performed by humans. It begins before the system test and integration life cycle phase. O\&SHA identifies safety requirements necessary to eliminate hazards or mitigate the risk of hazards.

These hazard analyses will identify certain hazards. The table in Figure 22 suggests broad classes of hazards that may be present. The various system hazard analyses will attempt to eliminate as many hazards as possible, reduce the probability of occurrence of those that remain, and reduce the potential damage which may result from accidents. In the latter two cases, responsibility will be assigned to specific system components for the control of occurrences and consequences. In some cases, software components may be assigned such responsibility. If this occurs, software hazard analysis is a form of component hazard analysis. 


\begin{tabular}{|ll|}
\hline a. & Occurrence of design bases conditions identified in the Safety Analysis Report. \\
\hline b. & $\begin{array}{l}\text { Possible independent, dependent and simultaneous ACE events considering failures of } \\
\text { safety equipment. }\end{array}$ \\
\hline c. & Interface considerations among various elements of the system. \\
\hline d. & Environmental constraints. \\
\hline e. & Operating, test, maintenance and emergency procedures. \\
\hline f. & $\begin{array}{l}\text { Design and use of test and maintenance equipment that has potential for introducing } \\
\text { damage, software errors or interrupts. }\end{array}$ \\
\hline g. & Safety equipment design and possible alternate approaches. \\
\hline h. & $\begin{array}{l}\text { Degradation in a subsystem or the total system from normal operation of another } \\
\text { subsystem including non-safety systems. }\end{array}$ \\
\hline i. & $\begin{array}{l}\text { Modes of failure, including reasonable human errors as well as single point failures, and } \\
\text { ACEs created when failures occur in subsystem components. }\end{array}$ \\
\hline j. & Potential contribution of software, events, faults and occurrences on safety of the system. \\
\hline k. & Potential common mode failures. \\
\hline l. & $\begin{array}{l}\text { The method of implementation of the software design requirements and corrective actions } \\
\text { will not impair or degrade the safety system nor introduce new ACEs. }\end{array}$ \\
\hline m. & $\begin{array}{l}\text { The method of controlling design changes during and after system acceptance will not } \\
\text { degrade the safety system nor introduce new ACEs. }\end{array}$ \\
\hline
\end{tabular}

Figure 17. Summary of Safety System ACEs Identification

a. Software requirements should be evaluated to identify those that are essential to accomplishing the safety function. These critical requirements should be evaluated against the ACE to assess their significance.

b. Requirements for timing and sizing should be included to ensure adequate resources for execution time, clock time and memory allocations are provided to support the critical requirements.

c, In designs involving the integration of multiple software systems, consideration should be given for interdependencies and interactions between the components of the system.

d. Existing software should be evaluated to ensure adequate confidence that no "unintended functions" detrimental to the operation of the safety system are introduced.

Figure 18. Summary of Software Requirements ACEs Identification 


\begin{tabular}{|ll|}
\hline a. & Equations, algorithms and control logic should be evaluated for potential problems. \\
\hline b. & Potential computational problems should be evaluated. \\
\hline c. & Evaluation of data structure and intended use should be performed. \\
\hline d. & Potential data handling problems should be evaluated. \\
\hline e. & Interface design considerations should be reviewed. \\
\hline g. & Adequate confidence that the design fits within the identified system constraints. \\
\hline h. & Software modules that implement critical functions should be identified. \\
\hline i. & $\begin{array}{l}\text { Non-safety modules should be evaluated to provide adequate confidence that they do not } \\
\text { adversely affect safety software. }\end{array}$ \\
\hline
\end{tabular}

Figure 19. Summary of Software Design ACEs Identification

\begin{tabular}{|ll|}
\hline a. & Evaluate equations, algorithms and control logic for potential problems. \\
\hline b. & $\begin{array}{l}\text { Confirm the correctness of algorithms, accuracy, precision and equation discontinuities, } \\
\text { out of range conditions, breakpoints, erroneous inputs, etc. }\end{array}$ \\
\hline c. & $\begin{array}{l}\text { Evaluate the data structure and usage in the code to provide adequate confidence that the } \\
\text { data items are defined and used properly. }\end{array}$ \\
\hline d. & $\begin{array}{l}\text { Provide adequate interface compatibility of software modules with each other and with } \\
\text { external hardware and software. }\end{array}$ \\
\hline e. & Provide adequate confidence that the software operates within the imposed constraints. \\
\hline f. & $\begin{array}{l}\text { Examine non-critical code to provide adequate confidence that it does not adversely } \\
\text { affect the function of critical software. }\end{array}$ \\
\hline g. & $\begin{array}{l}\text { Provide adequate confidence that the results of coding activities are within the timing and } \\
\text { sizing constraints. }\end{array}$ \\
\hline
\end{tabular}

Figure 20. Summary of Software Code ACEs Identification 


\begin{tabular}{|ll|}
\hline a. & Eliminate identified ACEs or reduce associated risk through design, if possible. \\
\hline b. & $\begin{array}{l}\text { Ensure that the safety functions are protected from identified ACEs, and that non-safety } \\
\text { functions do not create ACEs for the safety functions. }\end{array}$ \\
\hline c. & $\begin{array}{l}\text { Identify, evaluate and eliminate ACEs associated with each system throughout the entire } \\
\text { life cycle of a system. }\end{array}$ \\
\hline d. & Minimize risk resulting from excessive environmental conditions. \\
\hline e. & $\begin{array}{l}\text { Design to minimize risk created by human error in the operation and support of the } \\
\text { system. }\end{array}$ \\
\hline f. & $\begin{array}{l}\text { Create unambiguous requirements definitions to minimize the probability of } \\
\text { misinterpretation by developers. }\end{array}$ \\
\hline g. & Consider and use historical ACEs data, including lessons learned from other systems. \\
\hline h. & Minimize risk by using existing designs and test techniques whenever possible. \\
\hline i. & $\begin{array}{l}\text { Analyze for ACEs and document changes in design, configuration or system } \\
\text { requirements. }\end{array}$ \\
\hline j. & Document identified ACEs and their resolution. \\
\hline
\end{tabular}

Figure 21. Summary of General Guidelines for ACE Resolution 


\begin{tabular}{|l|l|}
\hline Acceleration and motion & Leakage \\
\hline Chemical reactions & Moisture \\
\hline Dissociation & High humidity \\
\hline Oxidation & Low humidity \\
\hline Replacement & Power source failure \\
\hline Contamination & Pressure \\
\hline Corrosion & High pressure \\
\hline Electrical & Low pressure \\
\hline System failure & Changes \\
\hline Inadvertent activation & Radiation \\
\hline Shock & Thermal \\
\hline Thermal & Electromagnetic \\
\hline Explosion & Ionizing \\
\hline Fire & Ultraviolet \\
\hline Heat and temperature & Structural damage or failure \\
\hline High temperature & Stress concentrations \\
\hline Low temperature & Stress reversals \\
\hline Changes & Toxicity \\
\hline Impact and shock & Vibration and noise \\
\hline
\end{tabular}

Figure 22. Classes of Hazards (Hammer 1972)

\section{A.3. NIST Review of Software Hazard Analyses}

This draft report, based primarily on Mil-Std $882 B$ (the predecessor of $882 \mathrm{C}$ ), lists three requirements for software hazard analysis. The Software Hazard Analysis should:

1. Respond to every hazard identified in the System Hazard Analysis.

2. Ensure that the operation of the software does not interfere with the safety goals or operation of the system.

3. Evaluate and mitigate how software could hinder the safety goals or operation of the system.
The report describes six different software hazard analyses. The following description is taken from the report.

1. Software Requirements Hazard Analysis (SwRHA) ensures that system safety requirements have been properly defined, and that they can be traced from the system requirements to the software requirements; software design; and operator, user and diagnostic manuals. It begins during the requirements phase of the system life cycle. The PHL and PHA are inputs to this analysis. SwRHA examines the system requirements, software requirements and software design by reviewing system and software requirements documentation and program documentation. Recommendations and design and test requirements are incorporated into the Software Design 
Documents and the Software Test Plan. The results of the SwRHA are presented at the System Requirements Review (draft), System Design Review (update) and Software Requirements Review (final).

2. Software Design Hazard Analysis (SwDHA) identifies safety-critical software components that require analysis beyond design. It starts after the Software Requirements Review and should be mostly completed before starting software coding. The PHA, SHA and SwRHA are inputs to this analysis. SwDHA defines and analyzes safety critical software components (e.g., assessing their degree of risk and relationships to other components) and the design and test plan (e.g., ensuring safety requirements are properly defined in the design). Changes are made to the Software Design Document (to eliminate hazards or mitigate the risk of hazards), and safety requirements are integrated into the Software Test Plan. Recommendations are made for coding. The results of the SwDHA are presented at the Software Design Review.

3. Software Code Hazard Analysis (SwCHA) identifies how to eliminate hazards or mitigate the risk of hazards during the coding phase of the life cycle. It starts at the beginning of that phase and continues until after system testing has been completed. The SwDHA is the input to this analysis.

SwCHA analyzes the actual source and object code, system interfaces, and software documentation (to ensure safety requirements are included).

Recommendations are made to change the software design, code and software testing. The results of the SwCHA are presented at the Test Readiness Review. (SwCHA results for lower level units are given to programmers during coding.)

4. The purpose of Software Safety Testing is to determine that all hazards have been eliminated or that each hazard's risk has been mitigated. Software Safety Testing of lower-level units starts very soon after their coding is completed. Software Safety

Testing tests safety-critical software components under normal conditions and under abnormal environment and input conditions. It also ensures that the software performs correctly and safely under system testing. Software Safety Testing includes testing of any commercial or government furnished software present in the system. The results of Software Safety Testing is to identify corrections to the software which, when implemented, will eliminate hazards or mitigate the risk of hazards. Retests are then performed on the corrected software under the same conditions. Testing of the software at the system level starts following a successful Test Readiness Review.

5. The Software/User Interface Analysis manages hazards that were not eliminated or controlled in the system design phase. For example, change recommendations are made to the design that provide hazard detection and operator warning, hazard recovery, and event or process termination.

6. Software Change Hazard Analysis analyzes all changes made to the software to determine their impact on safety. Software hazard analysis and testing is performed on all changes made to the requirements, design, code, systems, equipment, and test documentation to ensure that the changes do not create new hazards or affect existing hazards and that the change is properly incorporated into the code.

\section{A.4. Review of the Published Literature}

The published literature on software hazard analysis is sparse and recent, except for the application of fault trees to software. Some general background can be found in (Brown 1985; Leveson 1991b; Gowen 1992; and Elahi 1993).

Leveson (1991a) proposes to augment traditional software engineering by a form of hazard analysis; this idea forms the basis for the approach proposed in this report. 
The use of fault trees to analyze software has received considerable attention. The following may be consulted: Leveson 1983; Fryer 1985; Connolly 1989; Lal-Gabe 1990; Bowman 1991; Leveson 1991a; Leveson 1991c; McKinlay 1991; Levan 1992; Clarke 1993; and Levinson 1993. Much of this, however, uses fault trees to analyze code for correctness. There are two difficulties with this approach, and it is not recommended in this report. First, the most important decisions that may affect hazards occur early in the life cycle, when the requirements are specified and the basic computer system architecture is chosen. A fault tree constructed after this is done is likely to overlook the early decisions, resulting in missed opportunities for improvement. Second, a fault tree carried to the program language level is likely to be very large, making it hard to analyze. There is also the temptation to concentrate on the leaves (statements), missing the important intermediate nodes of the tree that capture combinations of events that can lead to failures.

The use of fault trees early in the software development process can be quite useful, particularly if they are an extension of fault trees developed for the overall reactor. They should probably be restricted to analysis, since the assignment of failure probabilities to software architectural elements is very problematic.

A few articles discuss other techniques. Leveson (1991a) also includes a discussion of Petri nets and state charts, and Mojdehbakhsh (1994) includes Statemate charts within a discussion of fault trees. Levinson (1993) includes fault trees, failure modes and effects analysis (FMEA) and Parts Stress Analysis (PSA).

Two articles were most influential on the development of this report, both by McDermid $(1994,1995)$. The first proposes the use of HAZOP and guide words to structure software hazard analysis, while the latter describes experiences in carrying out the technique. This report extends the approach by McDermid, placing it into a broader plan for software hazard analysis, extending the list of guide words to cover many aspects of software, and specializing somewhat to the nuclear reactor industry. 


\section{APPENDIX B. POTENTIAL SOFTWARE SAFETY ANALYSIS METHODS}

The New Mexico chapter of the System Safety Society issued a report on safety analysis in 1993. The relevant portion of that report is a 312-page discussion of hazard analysis techniques. Ninety techniques are discussed to varying levels of detail. The following topics are included for each technique:

- alternate names

- purpose

- method

- application .

- thoroughness

- mastery required

- difficulty of application

- general comments and references

Many of the techniques do not apply directly to software (for example, Tornado Analysis). Some of the remaining analyses could have indirect application to software. Bent Pin Analysis, for example, applies to connector pins in a cable connection. If the cable carries computer data, a bent pin could affect software functions.

However, the analysis is performed on the cable, not the software, so it is considered to be indirect.

The 47 techniques that might potentially apply to software are listed below. The word "potential" means that it is conceivable that the technique could be used, not that there is any evidence of use. For each of these techniques, the list gives its name and an extract of the purpose. In some cases, the purpose sections were not very complete.

- Accident Analysis evaluates the effect of scenarios that develop into credible and incredible accidents. This is expected to be performed at the system level, but could be extended to software safety by considering the effect of software on the prevention, initiation or mitigation of accidents identified in the system accident analysis.
- Cause-Consequence Analysis combines the inductive reasoning features of Event Tree Analysis with deductive reasoning features of Fault Tree Analysis. The result is a technique that relates specific accident consequences to their many possible causes. Computer codes exist to assist in the performance of this analysis. GENII, RSAC4, MACCS, ARA, EPA-AIRDOS and HOTSPOT are examples.

- Change Analysis examines the potential effects of modifications from a starting point or baseline. The Change Analysis systematically hypothesizes worst-case effects from each modification from that baseline.

- Checklist Analysis uses a list of specific items to identify known types of hazards, design deficiencies and potential accident situations associated with common equipment and operations. The identified items are compared to appropriate standards.

- Common Cause Analysis identifies any accident sequences in which two or more events could occur as the result of a common event or causative mechanism.

- Comparison-To-Criteria (CTC) Analysis provides a formal and structured format that identifies all safety requirements for a (software) system and ensures compliance with those requirements.

- Contingency Analysis is a method of preparing for emergencies by identifying potential accident-causing conditions and respective mitigating measures to include protective systems and equipment.

- Critical Incident Technique uses historical information or personal experience in order to identify or determine hazardous conditions and high-risk practices.

- Criticality Analysis ranks each potential failure mode identified in a Failure Modes and Effects Analysis (FMEA) according to 
the combined influence of severity classification and its probability of occurrence based on the best available data. It is often combined with FMEA, forming a Failure Modes, Effects and Criticality Analysis (FMECA).

- Digraph Utilization Within System Safety is used to model failure effect scenarios within large complex systems, thereby modeling FMEA data. Digraphs can also be used to model hazardous events and reconstruct accident scenarios. As a result, both hazard analysis and accident investigation processes can be improved via modeling event sequences.

- Event and Casual Factor Charting reconstructs the event and develops root cause(s) associated with the event.

- Event Tree Analysis is an analytical tool that can be used to organize, characterize and quantify potential accidents in a methodical manner. An event tree models the sequence of events that results from a single initiating event.

- Failure Modes and Effects Analysis (FMEA) determines the result or effects of sub-element failures on a system operation and classifies each potential failure according to its severity.

- Failure Modes, Effects and Criticality Analysis (FMECA) tabulates a list of equipment in a process along with all of the possible failure modes for each item. The effect of each failure is evaluated.

- Fault Hazard Analysis is a basic inductive method of analysis used to perform an evaluation that starts with the most specific form of the system and integrates individual examinations into the total system evaluation. It is a subset of FMEA.

- Fault Isolation Methodology is applied to large hardware/software systems that are unmanned and computer-controlled. There are five specific methods: half-step search, sequential removal or replacement, mass replacement, lambda search and point of maximum signal concentration.

- Fault Tree Analysis (FTA) assesses a system by identifying a postulated undesirable end event and examines the range of potential events that could lead to that state or condition.

- Hazard and Operability Study (HAZOP) is a group review method that assesses the significance of each way a process element could malfunction or be incorrectly operated. The technique is essentially a structured brainstorming session using specific rules.

- Hardware/Software Safety Analysis examines an entire computer system so that the total system will operate at an acceptable level of risk.

- Human Error Analysis is used to identify the systems and the procedures of a process where the probability of human error is of concern. This technique systematically collects and analyzes the large quantities of information necessary to make human error assessments.

- Human Factors Analysis allocates functions, tasks and resources among humans and machines.

- Interface Analysis identifies potential hazards that could occur due to interface incompatibilities.

- Maximum Credible Accident/Worst-Case Analysis determines the upper bounds on a potential accident without regard to the probability of occurrence of the particular accident identified.

- Nuclear Safety Cross-Check Analysis (NSCCA) verifies and validates software designs. It is also a reliability hazard assessment method that is traceable to requirements-based testing.

- Petri Net Analysis provides a technique to model system components at a wide range of abstraction levels. It is particularly useful 
in modeling interactions of concurrent components. There are many other applications.

- Preliminary Hazard Analysis (PHA) can be used in the early stages of system design (possibly including software design), thus saving time and money which could have been required for major redesign if the hazards were discovered at a later date.

- Preliminary Hazard List (PHL) creates a list of hazards to enable management to choose any hazardous areas to place management emphasis.

- Probabilistic Risk Assessment (PRA) provides an analysis technique for low probability, but catastrophically severe events. It identifies and delineates the combinations of events that, if they occur, will lead to an accident and an estimate of the frequency of occurrence for each combination of events, and then estimates the consequences.

- Production System Hazard Analysis identifies (1) potential hazards that may be introduced during the production phase of system development which could impair safety and (2) their means of control. This could apply to software if "production" is replaced by "operation."

- Prototype Development provides a modeling/simulation analysis technique that constructs early pre-production products so that the developer may inspect and test an early version.

- Repetitive Failure Analysis provides a systematic approach to address, evaluate and correct repetitive failures.

- Root Cause Analysis identifies causal factors relating to a mishap or near-miss incident. The technique goes beyond the direct causes to identify fundamental reasons for the fault or failure.

- Safety Review assesses a system or evaluates operator procedures for hazards in the design, the operations, or the associated maintenance.

- Scenario Analysis identifies and corrects potentially hazardous situations by postulating accident scenarios where credible and physically possible events could cause the accident.

- Sequentially-Timed Events Plot (STEP) Investigation System is a multi-linear events sequence-based analytical methodology used to define systems; analysis system operations to discover, assess and find problems; find and assess options to eliminate or control problems; monitor future performance; and investigate accidents. STEP results are consistent, efficiently produced, non-judgmental, descriptive and explanatory work products useful over a system's entire life cycle.

- Single-Point Failure Analysis identifies those failures that would produce a catastrophic event if they were to occur by themselves.

- Sneak-Circuit Analysis identifies unintended paths or control sequences that may result in undesired events or inappropriately timed events.

- Software Failure Modes and Effects Analysis (SFMEA) identifies softwarerelated design deficiencies through analysis of process flow charting. It also identifies interest areas for verification/validation and test and evaluation.

- Software Fault Tree Analysis applies FTA to software. It can be applied to design or code.

- Software Hazard Analysis identifies, evaluates and eliminates or mitigates software hazards by means of a structured analytical approach that is integrated into the software development process.

- Software Sneak Circuit Analysis (SSCA) is used to discover program logic that could cause undesired program outputs or inhibits, or incorrect sequencing/timing. 
- Subsystem Hazard Analysis (SSHA) identifies hazards and their effects that may occur as a result of the design of a subsystem.

- System Hazard Analysis (SHA) concatenates and assimilates the results of Subsystem Hazard Analyses into a single analysis to ensure that hazards or their controls or monitors are elevated to a system level and handled as intended.

- Systematic Inspection uses checklists, codes, regulations, industry consensus standards and guidelines, prior mishap experience and common sense to methodically examine a design, system or process in order to identify discrepancies representing hazards.
- Uncertainty Analysis identifies the incertitude of a result based on the confidence levels (or lack thereof) and variability associated with the inputs.

- What-If Analysis is a brainstorming approach in which a group of experienced individuals asks questions or voices concerns about possible undesired events in a process.

- What-If/Checklist Analysis is a combination of What-If Analysis and Checklist Analysis. 


\section{APPENDIX C. SOFTWARE TOOLS FOR HAZARD ANALYSIS}

Hazard analysis in general, and software hazard analysis in particular, can be much assisted by the use of convenient software tools. Many tools are available that address different aspects of hazard analysis and run on different platforms. Costs vary from free to tens of thousands of dollars. Capabilities and quality also vary considerably. Platforms include PC, Macintosh, Sun and other Unix, VAX and other systems.

A small sample of tools was examined as an indication of the types of tools available. The sampling was restricted to tools that use the PC as a platform (running either under MS-DOS or Windows 3.x), and tools that cost less than $\$ 500.00$. Only one example from each type of analysis was examined. Results are indicative, but are not necessarily representative of the tools available in the marketplace. Tool revisions are frequent, so the vendors should be contacted directly for current release information. No endorsement of any tool is implied by this study.

Six subject areas were used in the study:

- Fault tree analysis (FTA)

- Failure modes, effects and criticality analysis (FMEA and FMECA)

- HAZOP

- Hazard tracking

- Markov chain modeling

- Reliability growth analysis

The remainder of this appendix describes the various programs that were investigated. Each section begins with a brief description of the program: program name, vendor, platform and primary functions. A description of the program's capabilities follows, with illustrations of the reports that may be produced. No attempt is made here to discuss the various techniques (see Lawrence (1993) and the references given there for background). Opinions expressed are those of the author, and apply only to the software versions actually examined. Most of the versions examined had minor faults; these are not discussed. Some major problems are given for a few of the programs when these appeared to create considerable difficulty in using the program.

\section{C.1. Fault Tree Analysis}

$\begin{array}{ll}\text { Product Name: } & \text { FaulTrEase } \\ \text { Product Vendor: } & \begin{array}{l}\text { Arthur D. Little, Inc., } \\ \text { Cambridge, MA. }\end{array} \\ \text { Platform: } & \begin{array}{l}\text { Windows 3.1. (A } \\ \text { Macintosh version is } \\ \text { available) }\end{array}\end{array}$

FaulTrEase is a program for drawing and evaluating fault trees. The program provides considerable help in drawing the trees, since it calculates the proper position of the entire tree as nodes and branches are added or deleted. As a result, a fault tree can be constructed with considerable ease by concentrating on the contents of nodes and the connections between them. Building fault trees using this program is quite efficient.

Calculations include cut sets, probability calculations and calculation of importance.

The program is not able to handle n-out-of-m nodes, which hampers use for analysis of reactor protection systems, where it is desirable to include 2-out-of-4 and similar logics. Printing is limited to a single sheet, even though the program knows that several sheets are required in order to print the full tree. This makes large fault trees difficult to document. The solution is to divide the tree into separate subtrees, perform calculations on the latter, and manually insert the results into the main tree. This is subject to copying errors, and is quite inconvenient.

The figures show an example using fault trees for the AP600 reactor design. The probability data shown is that used in the AP600 fault trees when available; estimates are used when AP600 data was not given.

The approach used was to copy the fault trees from material provided by the NRC Program Monitor. Options in the program permit tree layouts to be compressed to save space, or 
expanded for better appearance. Both options are illustrated in the examples. Probability values are assigned to leaves of the tree and the probability associated with the top node of the tree can be calculated by the program. If subtrees are used, they are evaluated first, and then the value associated with the top node of the subtree is manually entered into the corresponding off-page connector of the main tree. For example, the first page shows the toplevel tree, as printed by the program. Off-page connecting nodes labeled 8,11,22 and 24 are all similar, and use the results of the second tree, "AP600 Failure to Actuate Valve" tree. This tree requires two printed pages since it's too large to fit on a single sheet; off-page connector " $B$ " is used to connect the sheets.

Some additional lower-level charts are illustrated on succeeding pages.

\section{C.2. FMEA, FMECA, HAZOP}

Product Name: HAZOPtimizer

Product Vendor: $\quad$ Arthur D. Little, Inc., Cambridge, MA.

Platform: $\quad$ DOS 5.x or Windows 3.1.

HAZOPtimizer is used to organize, store and print tabular reports used for various types of hazard analysis. Report columns can be named, defined and arranged to suit the needs of the user. The product gives the appearance of a semi-structured interface to a database.

The primary unit of data collection is termed a study — which documents the results of a particular analysis on a particular problem. Typical analyses include FMEA, FMECA, HAZOP and PHA. Some pre-defined templates exist, but were not found useful.

Study results are organized into sheets. Each sheet has the same definition of data columns; the use of multiple sheets is for convenience. A sheet contains lines, upon which the study results are entered.

The figures show two sample studies. The first is an FMECA study from the book Guidelines for Hazard Evaluation Procedures, Center for
Chemical Process Safety, 1992, page 207. The second uses data from a 1992 evaluation of the GE ABWR design performed by LLNL for NRC/NRR. Only a small portion of that study is included.

The examples illustrate the flexibility of the program, since different column definitions were used by the two different sources.

The program limits each box in the tables to 256 characters; this appears to be a limitation inherited from the underlying database management system, and was found to be extremely inconvenient.

\section{C.3. Hazard Tracking}

Product Name: HazTrac

Product Vendor: Hoes Engineering, Davis, CA.

Platform: $\quad$ DOS 5.x or Windows 3.1.

HazTrac assists in carrying out a hazard analysis. It is considerably more structured than HAZOPtimizer, and is organized to meet the requirements of Mil Std 882B. HazTrac can be used to support the analyses specified therein: PHA, SHA, SSHA, FMEA and OSHA. (There is also an option specific to the State of California, which is not discussed here.)

Information is organized into three levels: hazard, recommendations and status. There is an entry at the first level for each defined hazard. This depends on the type of analysis; a PHA, for example, records the scenario under which the hazard may occur, the potential effects and an assessment of risk. The latter use the Mil Std tables shown earlier in Figures 12 and 13.

The second level permits the recording of recommendations on eliminating or mitigating the hazard. The program records the recommendations and some associated information, including names of people and organizations responsible for the recommendation and due dates.

The third level permits tracking of changes in the status of each recommendation. This includes the current status (open, agreed, 
dropped or verified) and a historical log of status events.

This program is considerably easier to use than HAZOPtimizer, but restricts the user to the built-in forms. That is, ease of use comes at the expense of flexibility. Text fields are also limited to 256 characters, which remains troublesome. A particularly irritating feature is the restriction that the program and data must reside on different disks. The computer used for examining the program has only a single hard disk, so the HazTrac data was placed on a floppy disk. No logical reason for this requirement was known.

An example of a PHA is shown in the later figures. It shows a hypothetical analysis of a chlorine plant, taken from the book Guidelines for Hazard Evaluation Procedures, Center for Chemical Process Safety, 1992, pages 270-271.

\section{C.4. Markov Chain Modeling}

$\begin{array}{ll}\text { Product Name: } & \text { CARMS } \\ \text { Product Vendor: } & \text { Daina } \\ \text { Platform: } & \text { Windows } 3.1\end{array}$

CARMS is a general Markov modeling program. It can be used to draw a Markov model, assign probabilities to transitions and run a simulation. The latter provides a graph of the calculated probabilities of the various states through time, which provides the user with knowledge of how the state probabilities change with time, and how fast they move to a steady state.

A model is constructed by defining states, transitions between states, initial probabilities for the states, and transition probabilities. The latter can be defined using equations. Drawing the model is quite easy using the built-in capabilities.

CARMS can show the model as a drawing or as a table. Several examples are shown, giving the diagram and the results of the simulation. The screen display of the simulation shows labels for the various lines in the graph; they are not printed, however. To show this, the lines in the graph were annotated by hand below.
There's not much more to write about this program. It does one thing, and does it very nicely.

\section{C.5. Reliability Growth Modeling}

Product Name: CASRE.

$\begin{array}{ll}\text { Product Vendor: } & \text { Jet Propulsion Laboratory, } \\ & \text { Pasadena, CA. }\end{array}$

Platform: $\quad$ Windows 3.1.

Reliability growth modeling is a technique for predicting the reliability of a product undergoing test when the product is re-engineered after each failure. This pattern is well suited to software testing when each failure causes the underlying fault to be repaired and testing to be resumed with the modified program.

The primary product for modeling software reliability growth is SMERFS, a public domain package available from the Naval Surface Warfare Center. CASRE uses SMERFS, but has a window interface and several additional features.

CASRE can be used starting at any point during the testing of a module, program or software system. Failure data is recorded as testing and repair takes place. Two formats are possible: recording time units between each failure or recording the number of failures during each time interval.

The program analyzes the failure data in various ways and plots the results on the PC screen or a printer. Different models can be used to fit the data and make predictions. Several methods of displaying the results can be used; all are illustrated in the example below. This example uses a sample data set supplied with the product. Curve fitting is done using a Generalized Poisson model, with a prediction of future reliability shown as a line on the charts.

The final plot shows the same data fitted to two other models: the Schneidewind model and a Yamada S-shaped model. 


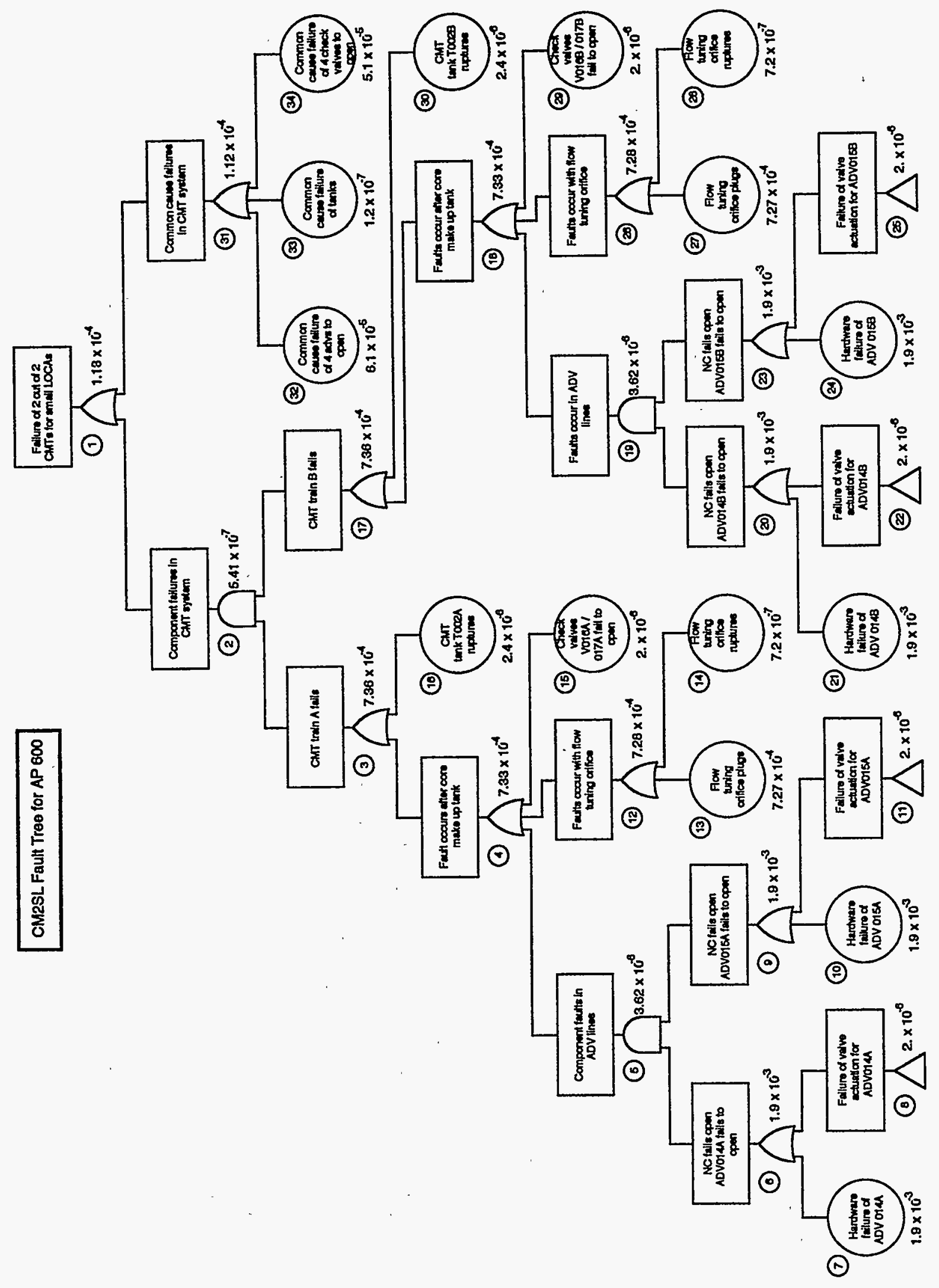




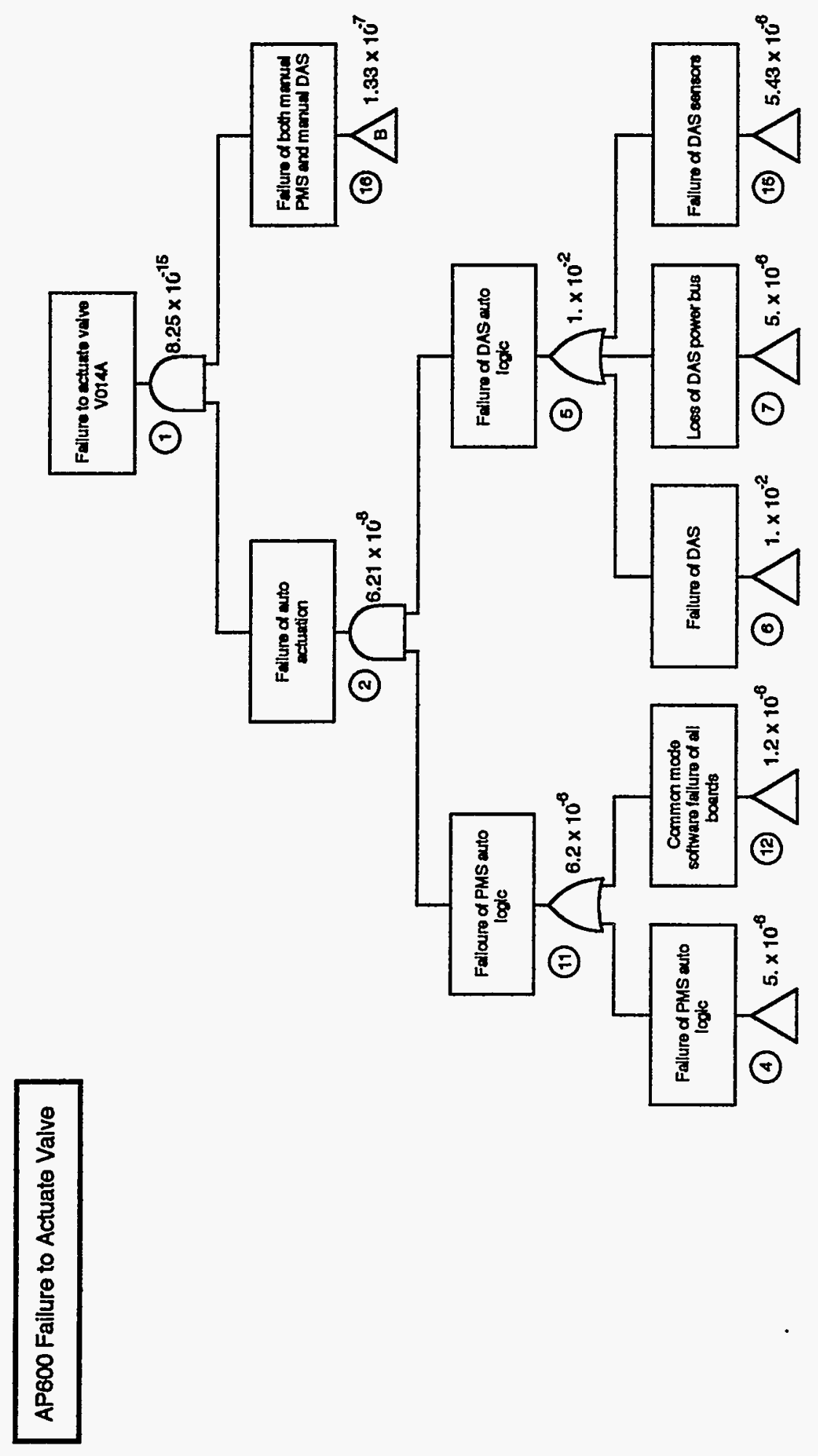




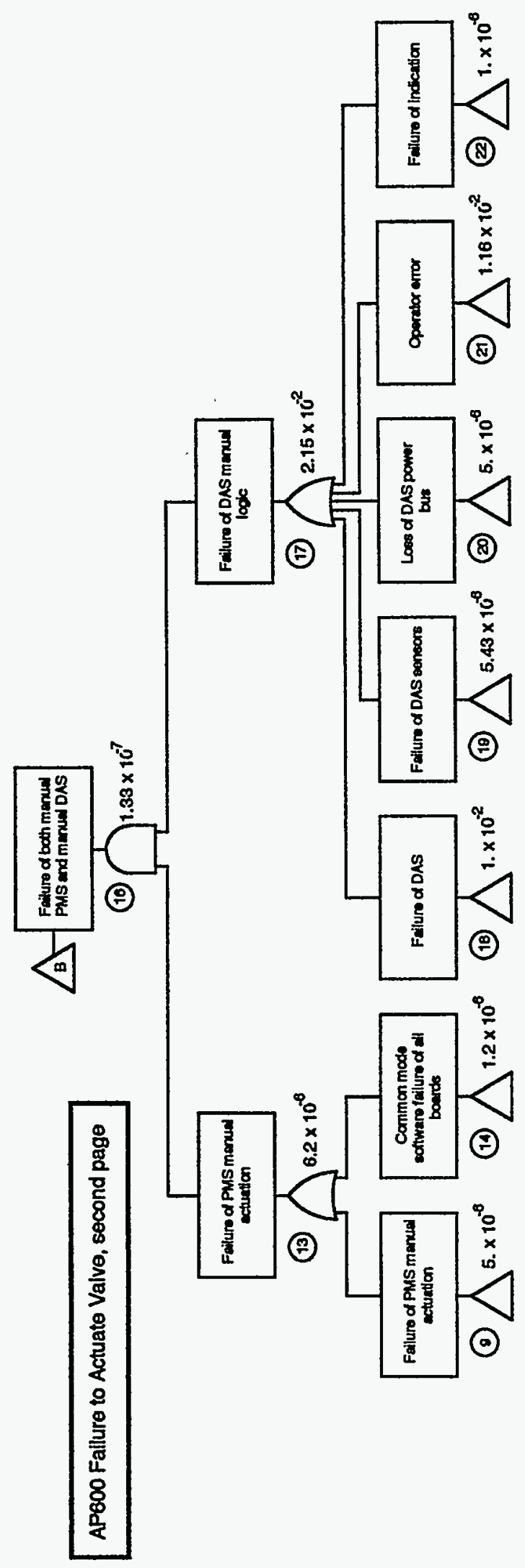



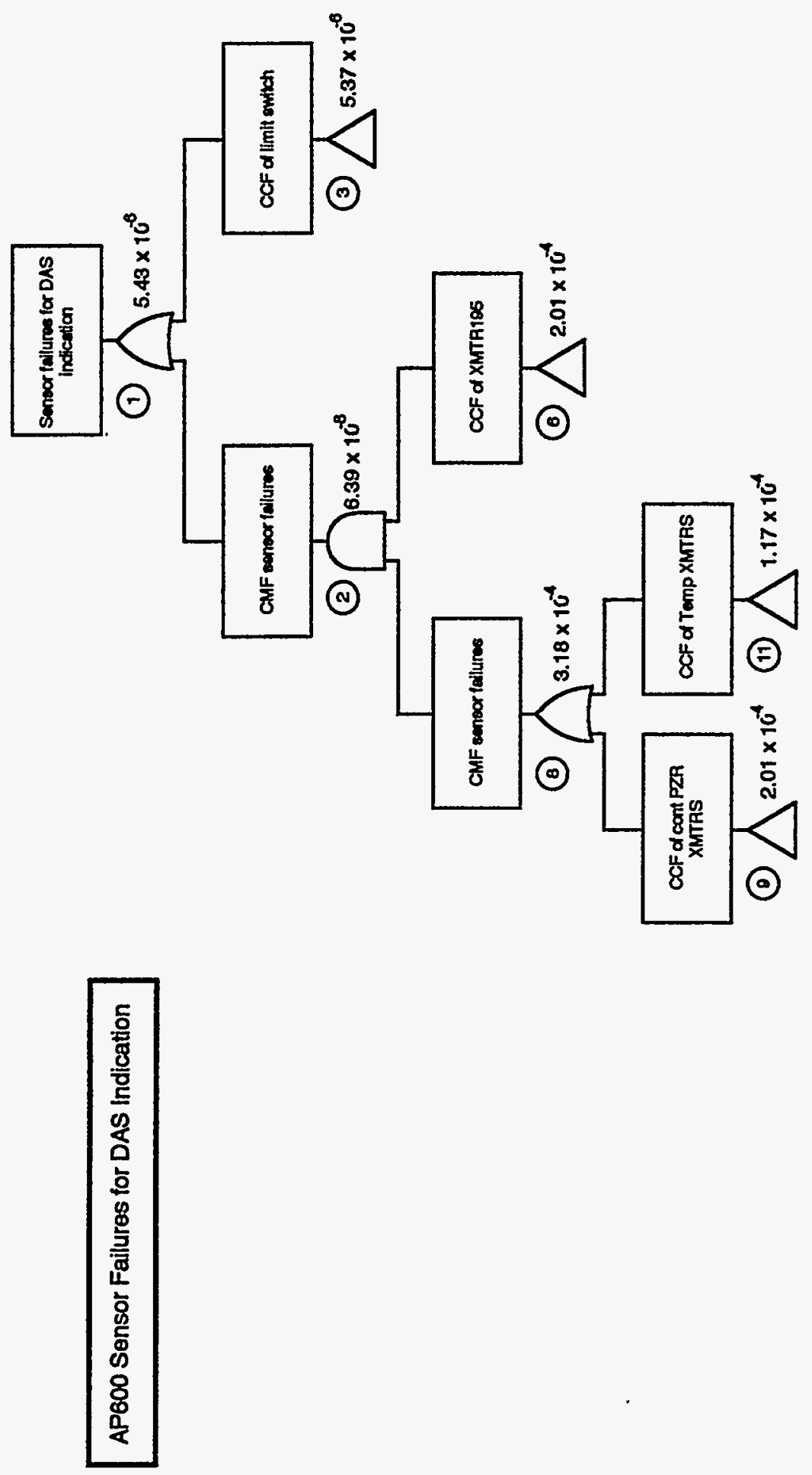

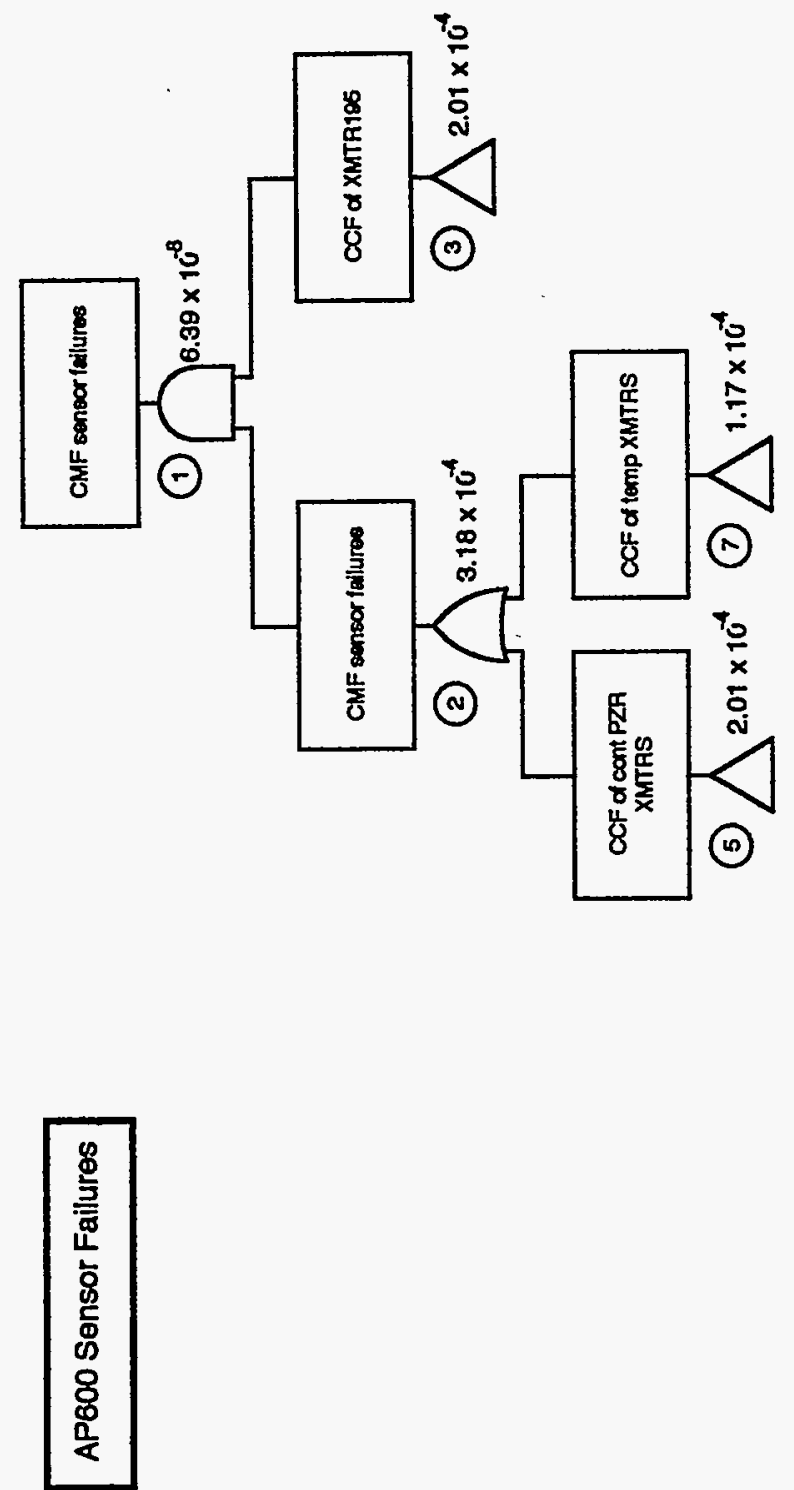

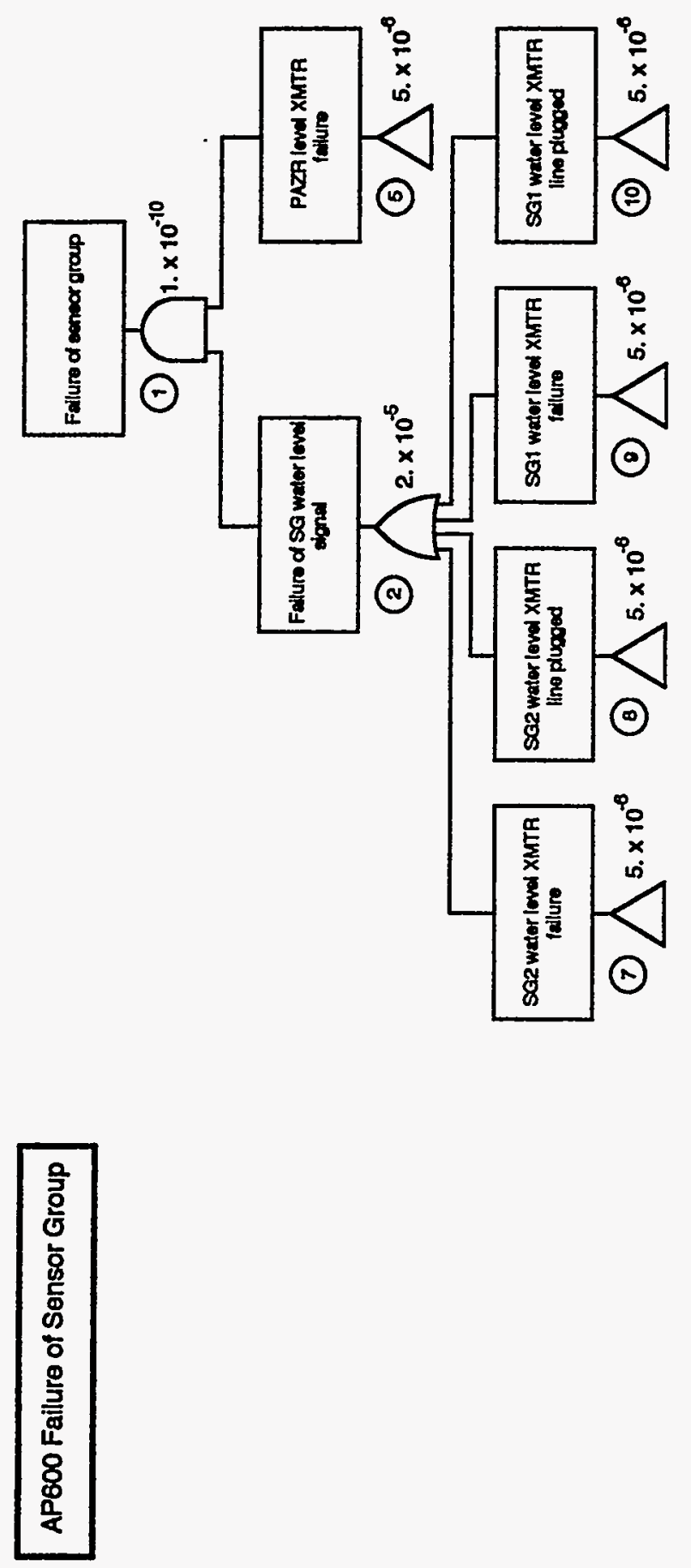
AP600 Sensor Failures

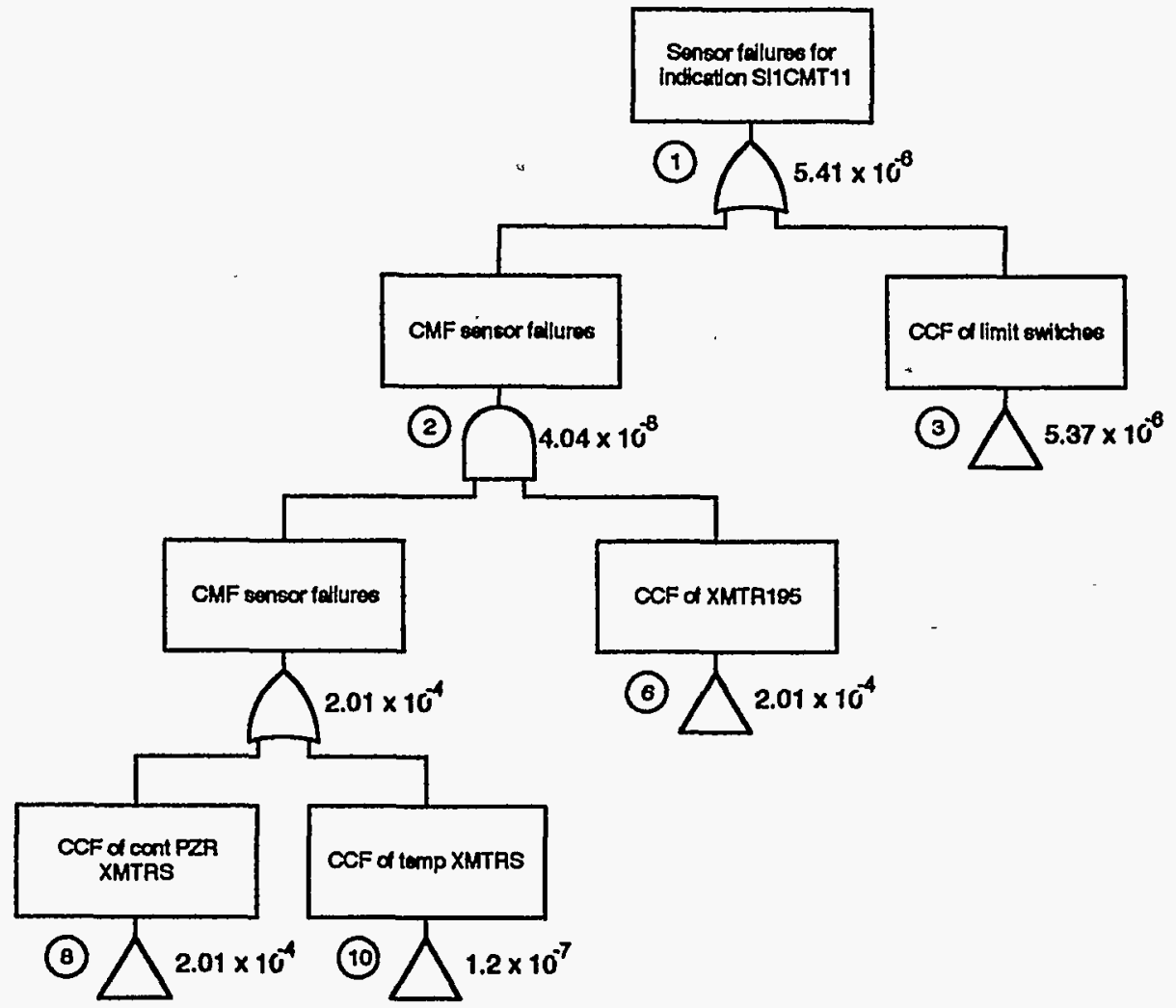


Hzzoptinizer by arthur D. Little, Inc.

Page 1

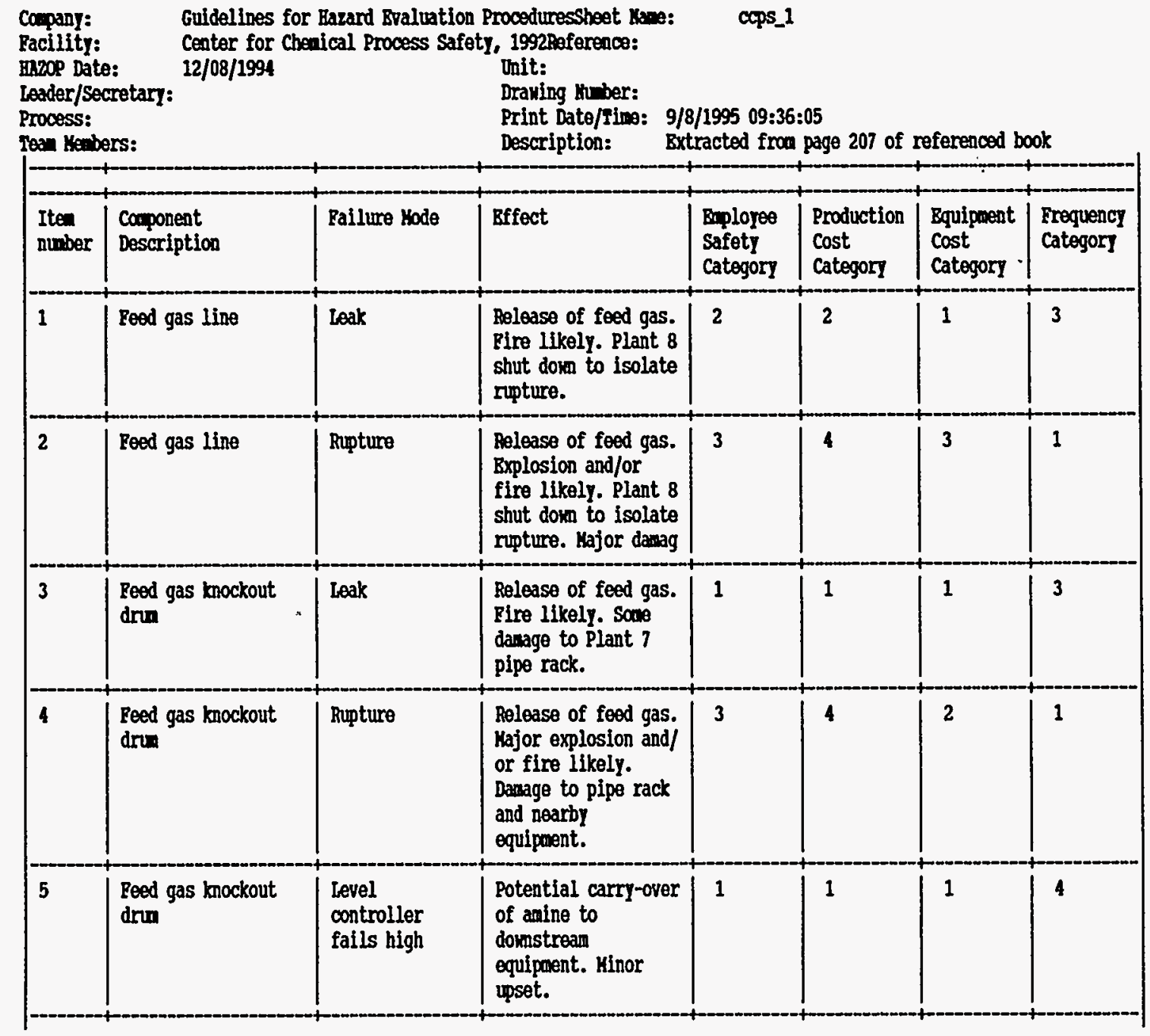


Hrzoptimizer by Arthur D. Little, Inc.

Page 2

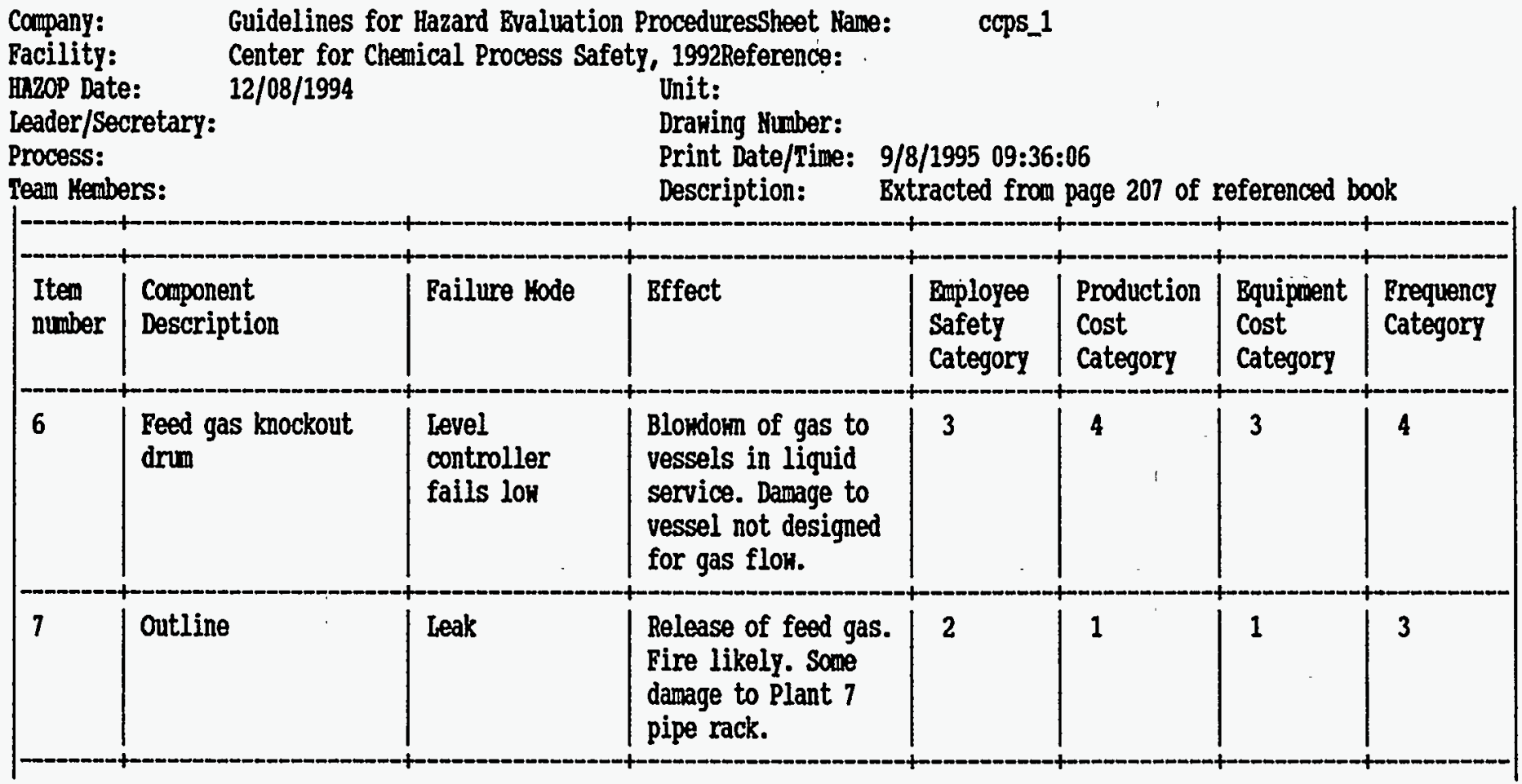


Hazoptinizer by Arthur D. Little, Inc.

\begin{tabular}{|c|c|c|c|c|c|c|}
\hline \multicolumn{2}{|c|}{$\begin{array}{ll}\text { Coupany: } & \text { LLK } \\
\text { Pacility: } & \text { GB } \\
\text { HiwOP Date: } & 12 / \\
\text { Leader/Secretary: } & \\
\text { Process: } & \\
\text { Tean Henbers: } & \end{array}$} & actor Design & $\begin{array}{l}\text { Sheot Mane: } \\
\text { Reference: } \\
\text { Unit: } \\
\text { Drauing Nunber: } \\
\text { Print Date/Time: } \\
\text { Description: }\end{array}$ & \multicolumn{2}{|c|}{$\begin{array}{l}\text { abur } \\
\text { LLIL Report to IRC, Sept. 7, } 1992 \\
9 / 8 / 199509: 40: 42\end{array}$} & \\
\hline $\begin{array}{l}\text { Iten } \\
\text { number }\end{array}$ & Component & Function & Bailure Hode & Inpact on System & Detection & Remarks \\
\hline 1 & 1LDS & $\begin{array}{l}\text { Uses various process } \\
\text { variables to detemine } \\
\text { if a loss of coolant is } \\
\text { occurring, and if so, it } \\
\text { then isolates the leak } \\
\text { from RPV. }\end{array}$ & $\begin{array}{l}\text { The IDS consists of } \\
\text { independent input } \\
\text { devices, software and } \\
\text { output devices contained } \\
\text { in other safety systens. } \\
\text { The input devices are } \\
\text { all quadruly redundant. } \\
\text { single failure of the } \\
\text { input devices bas no } \\
\text { effect on the successful } \\
\text { operation of uDS. }\end{array}$ & $\begin{array}{l}\text { to single failure can } \\
\text { completely disable IDS. }\end{array}$ & By surveillance. & \\
\hline 2 & MuS & $\begin{array}{l}\text { Takes in IPRN neutron } \\
\text { sensor values, averages } \\
\text { the values to get a } \\
\text { divisional average, } \\
\text { sends the divisional } \\
\text { average to other } \\
\text { divisions, takes in } \\
\text { other divisional } \\
\text { averages, and averages } \\
\text { divisional averages to } \\
\text { produce an APR trip } \\
\text { signal. }\end{array}$ & $\begin{array}{l}\text { The resultant average of } \\
\text { the divisional average } \\
\text { is incorrect and lower } \\
\text { than the actual reutron } \\
\text { flux. }\end{array}$ & $\begin{array}{l}\text { The resultant average of } \\
\text { the divisional averages } \\
\text { in the Aris for all } \\
\text { divisions is calculated } \\
\text { to be significantly less } \\
\text { than true value; reactor } \\
\text { will not trip on desired } \\
\text { APRA signal. }\end{array}$ & By surveillance. & $\begin{array}{l}\text { It is assued that range } \\
\text { checking uill be used. }\end{array}$ \\
\hline
\end{tabular}




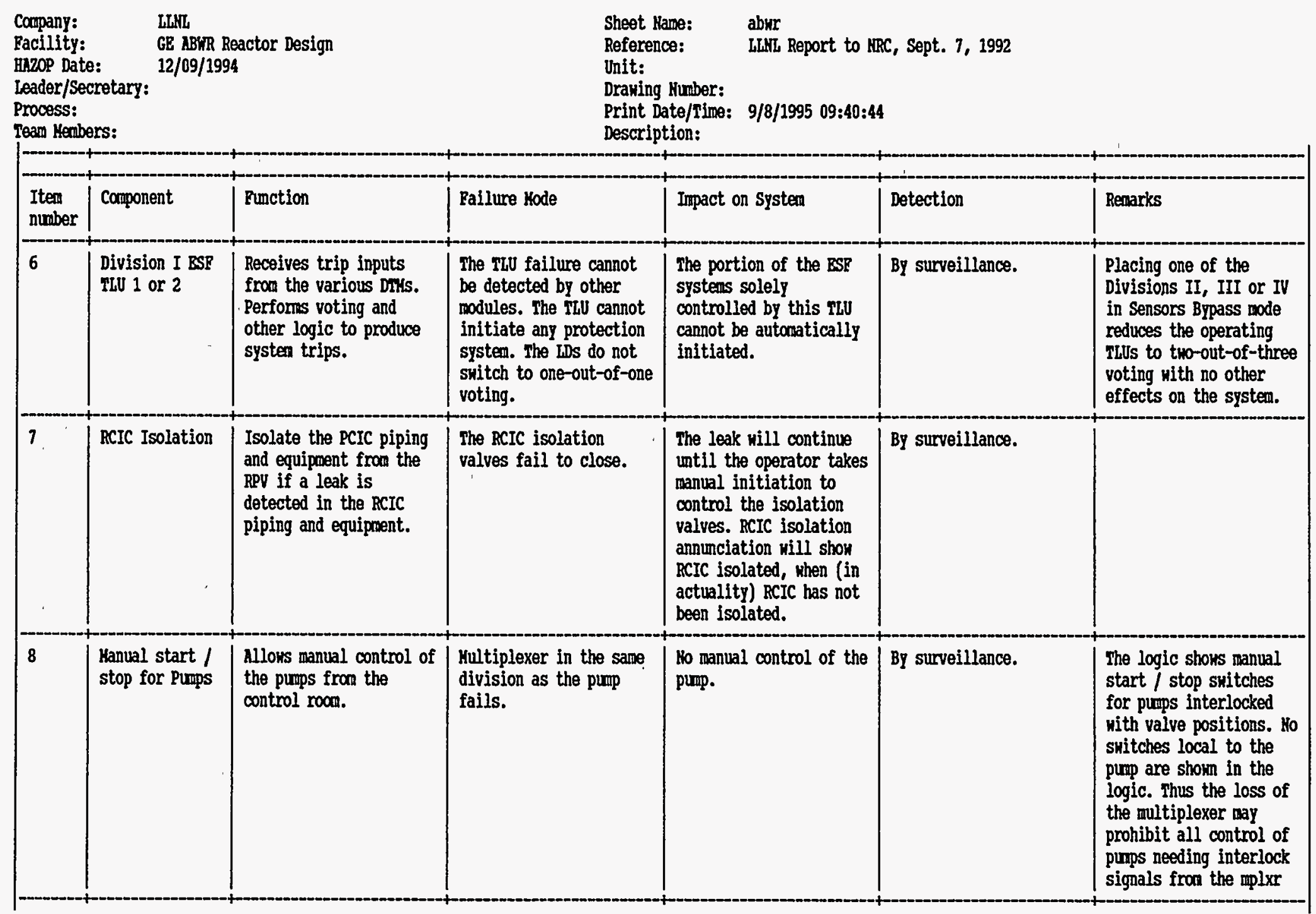


Hazoptimizer by Arthur D. Little, Inc.

Page 4

\begin{tabular}{|c|c|c|c|c|c|c|}
\hline \multicolumn{2}{|c|}{$\begin{array}{l}\text { Company: } \\
\text { Iacility: }\end{array}$} & $\begin{array}{l}\text { LLHT } \\
\text { GB IBWR Reactor Design } \\
12 / 09 / 1994\end{array}$ & $\begin{array}{l}\text { Sheet lame: } \\
\text { Reference: } \\
\text { Unit: } \\
\text { Drauing Number: } \\
\text { Print Date/Time: } \\
\text { Description: }\end{array}$ & $\begin{array}{ll}\text { me: } & \text { abur } \\
\text { e: } & \text { LLHL Report } \\
\text { Himber: } & \\
\text { te/Tine: } & 9 / 8 / 199509: \\
\text { ion: } & \end{array}$ & $\begin{array}{l}\text { abur } \\
\text { LLHL Report to KRC, Sept. 7, } 1992\end{array}$ & \\
\hline $\begin{array}{l}\text { Itend } \\
\text { number }\end{array}$ & Component & Function & Bailure Kode & Impact on Systen & Detection & Renarks \\
\hline 9 & $\begin{array}{l}\text { Diesel } \\
\text { Generators }\end{array}$ & $\begin{array}{l}\text { Provide electric power } \\
\text { to the protection system } \\
\text { equipwent when nomsal } \\
\text { power is lost. }\end{array}$ & $\begin{array}{l}\text { Loss of one diesel } \\
\text { generator or the ability } \\
\text { to initiate one } \\
\text { generator during LOCA. }\end{array}$ & Ho impact on systen. & By surveillance. & $\begin{array}{l}\text { GB documents shou the } \\
\text { diesel generators being } \\
\text { initiated automatically } \\
\text { from the protection } \\
\text { system during a loch, } \\
\text { but the logic on how } \\
\text { this occurs is } \\
\text { incomplete. }\end{array}$ \\
\hline
\end{tabular}




\section{PRELIMINARY HAZARD ANALYSIS \\ ABC Chemicals \\ VCM Plant Conceptual Design}

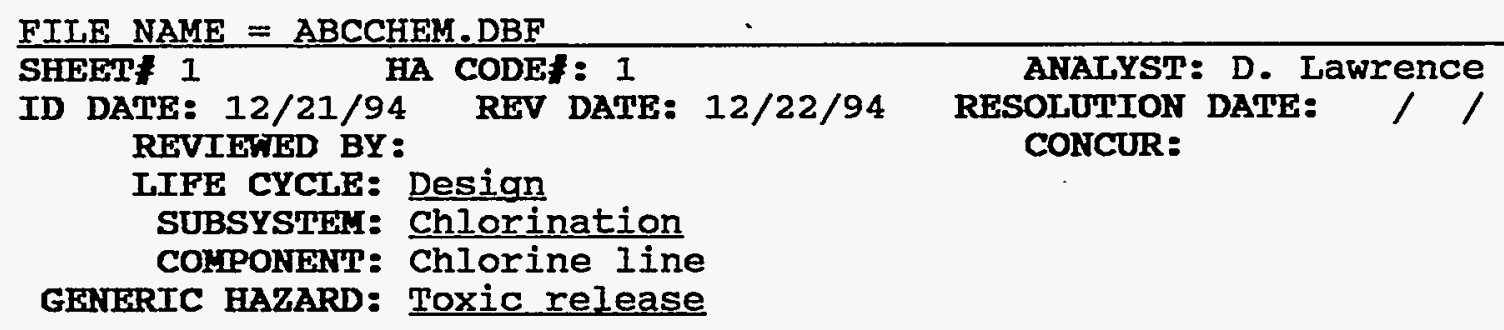

ANALYST: D. Lawrence

RESOLUTION DATE: / / CONCUR:

HAZARD SCENARIO:

Chlorine line gasket / packing leak

EFFECT:

Small chlorine release on site

RISR ASSESSHEHT:

SEVBRITY: IHITLAL RIHAL PROBABLLITY INITIAL FIHAL RAC CODRS:

I. CATASTROPHIC - - A. PREQUEMT - - - IHITIAL RAC 4

$\begin{array}{lllll}\text { II. CRITICAL - - } & \text { B. PROBABLE } & \overline{8} & - & \text { FIHAL RAC }\end{array}$

III. HARGIHAL
IV. HEGLIGIBLE
$\frac{1}{x}=$

REIMARKS OR COYAIENTS:

C. OCCASIOHAL

D. RRMOTB

B. ITPROBABLE 


\section{PRELIMINARY HAZARD ANALYSIS \\ ABC Chemicals \\ VCM Plant Conceptual Design}

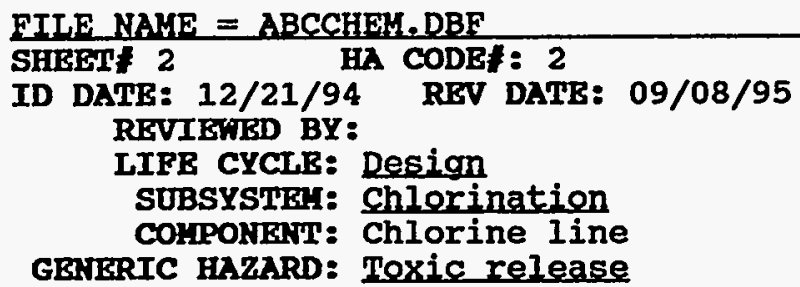

HAZARD SCEKARTO:

Chlorine line rupture (ie, vehicle accident, blocked-in line)

BRFBCT:

Large chlorine release, major on-site impact. Potential off-site

impacts.

RISK ASSESSI,IBIT:

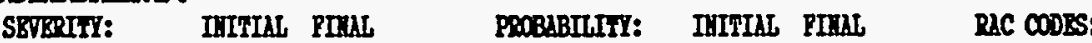

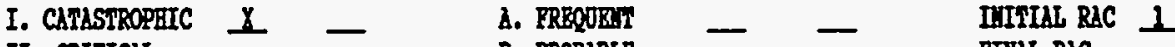

II. CRITICAL - - B. PROAABLB - - -

III. MARGIIAL - - - c. OCCASIOHAL $\bar{x}-$

$\begin{array}{ll}\text { IV. HEGLIGIBLE - }- \text { D. RR:OTB } & - \\ \text { B. INPROBABLE - - }\end{array}$

REMIRRRS OR COMATIITS:

RECONIFNDATIONS TO HINIIIZB RISK:

1) Verify chlorine line is evacuated whenever the var plant is dom for extended tire.

HA CODEF: 2

ACTION DUE: NOT REQ'D

POINT OF CONTACT:

RESPONSIBLE ORG:

REC CODE:

STATUS: DROPPED

Status History:

12/21/94 Recommendation identification date

01/14/95 Recommendation proved not feasible; dropped. 
2) Provide valyes and interlocks to positively isolate the line in the event of a rupture.

HA CODEF:- 2 ACTION DUE: / /

POINT OF CONTACT: RESPONSIBLE ORG:

REC CODE:

STATUS: OPEN

Status History:

12/21/94 Recommendation identification date

3) Train VGr plant personnel to respond to chlorine releases.

HA CODE\#: 2

ACTION DUE: / /

POINT OF CONTACT:

RESPONSIBLE ORG:

REC CODE:

STATUS: OPEN

Status History:

12/21/94 Recommendation identification date

4) Bquip VCr plant personnel with PPB for chlorine.

HA CODE\#: 2

POINT OF CONTACT:

ACTION DUE: / /

REC CODE: RESPONSIBLE ORG:

STATUS: OPEN

Status History:

$12 / 21 / 94$ Recommendation identification date

5) Do not bury chlorine pipeline.

HA CODE\#: 2

POINT OF CONTACT:

ACTION DUE:

REC CODE:

RESPONSIBLE ORG:

STATUS: OPEN

Status History:

12/21/94 Recommendation identification date 


\section{PRELIMINARY HAZARD ANALYSIS}

ABC Chemicals

VCM Plant Conceptual Design

FTIE NAYE = ABCCHEH.DBF

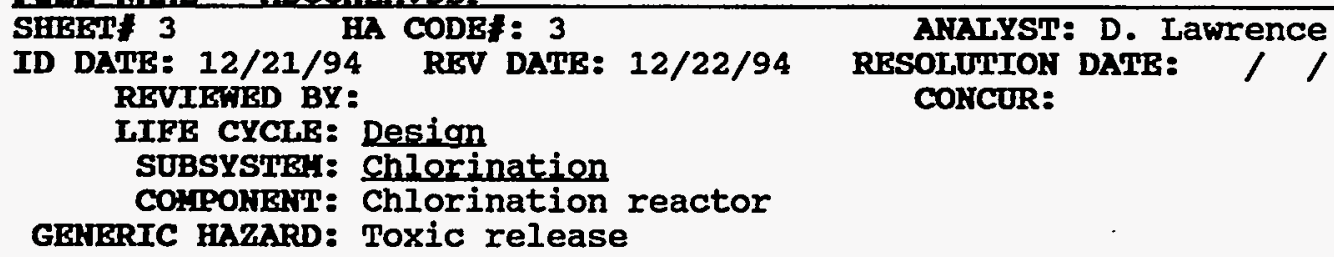

HAZARD SCEHARIO:

Direct clorination reactor exotherm

BFFBCT:

Large chlorine / EDC / ethylene release. Depending on reactor size and operating conditions, potential off-site impacts.

RISK ASSESSHIETT:

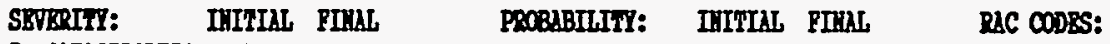

I. CATLSTROPHIC $\&$ a. PRBQURAT - - IITIAL RAC 2

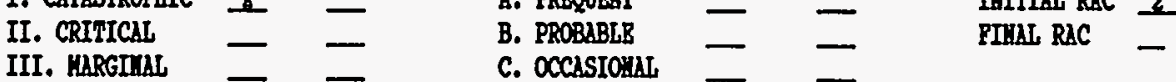

IV. IBGLIGIBLR

D. RR:OIR

B. IIPROBABLE

RKIIARKS OR COMTIEITS:

RECOMIRIDATIONS TO MIHIMIZB RISK:

1) Sonsider noving VCr plant rest of plant Road.

HA CODEF: 3

POINT OF CONTACT:

ACTION DUE: / /

REC CODE:

RESPONSIBLE ORG:

STATUS: OPEN

Status History:

12/21/94 Recommendation identification date

2) Perforn dispersion studies to assess off-site inpact of chlorine $/$ EDC release due to exothern.

HA CODEF: 3

POINT OF CONTACT:

ACTION DUE: / /

REC CODE:

RESPONSIBLE ORG:

STATUS: OPEN

status History: 
Appendix C. Figures: HazTrac

12/21/94 Recommendation identification date

3) Verify reactor pressure relief systen can handle this release.

HA CODEF: 3

POINT OF CONTACT:

ACTION DUE: / /

REC CODE:

STATUS: OPEN

Status History:

12/21/94 Recommendation identification date 
PRELIMINARY HAZARD ANALYSIS

ABC Chemicals

VCM Plant Conceptual Design

FTLE NAME = ABCCHEM,DBF

SHEET 4 HA CODE : 4

ID DATB: $12 / 21 / 94$ REV DATE: $12 / 22 / 94$

REVIETED BY:

LIFE CYCLB: Desion

SUBSYSTE: Chlorination

COYPONEKT: Chlorination reactor

GBHERIC HAZARD: TOXic release

HAZARD SCEIARIO:

Direct chlorination reactor rupture.

BFFECT:

Large chlorine / EDC / ethylene release. Depending on reactor size and operating conditions, potential off-site impacts.

RISK ASSESSIFEIT:

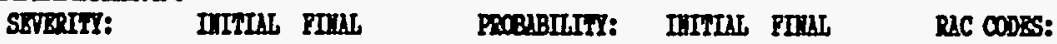

I. CATASTROPHIC $\perp-$ A. BRBQURAT - - IHITLAL RAC 2

II. CRITICAL - - B. PROAABLB - - C PCARIOLAL RAC -

III. MARGIUNI

IV. MEGLIGIBLB -

REITARRS OR COHIFITIS:

C. OCChSTOMLI

D. RERTORB

AKALYST: D. Lawrence

RBSOLUTION DATE: / / CONCUR:

B. IIPROBABLB — -

RBCOIIENDATIONS TO MIHIHIZB RISR:

1) Kininize inventory of chlorine / EDC in reactor.

HA CODE : 4

POINT OF CONTACT:

ACTION DUE:

REC CODE: RESPONSIBLE ORG

STATUS: OPEN

Status History:

12/21/94 Recommendation identification date 
PRELIMINARY HAZARD ANALYSIS

ABC Chemicals

VCM Plant Conceptual Design

FILE NAME = ABCCHEY .DBF

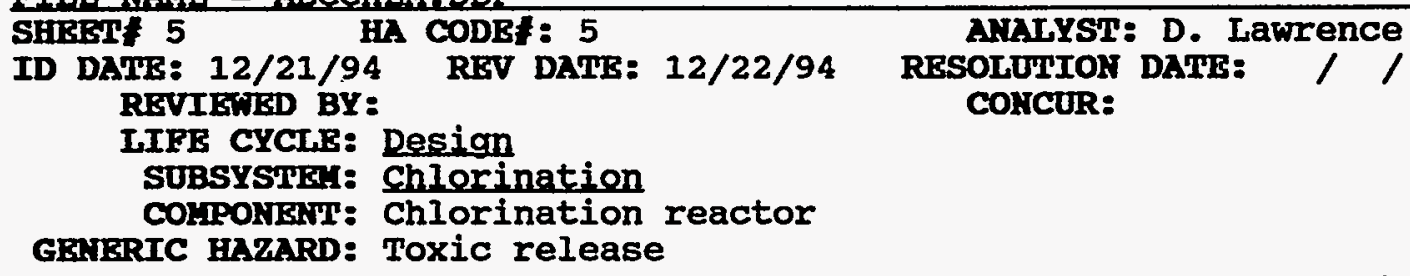

HAZARD SCEMARIO:

Direct chlorination reactor relief valve lift.

RFFBCT:

Potential large EDC / chlorine / ethylene release.

RISR ASSESSHEHT:

SETZPITI:

PROBABILITI: ITITLAL PIHLL

RAC CODRS:

I. CAPASTROPHII

II. CRITICAL

III. MARGIIAL

- -

A. IREQUESTI

B. PROBABLE

C. OCCASIOHALL

$\bar{x}-$

IHITLLL RAC 1

IV. HEGLIGIBLB

REIRRRS OR COMNHITS:

D. RRI:PTE

B. ITPROBABLR

PINAL RAC

\section{RECOHYIKIDATIONS TO KINIKIIZE RISK:}

1) Yerify the reactor pressure relief systen incinerator and scrubber are sired to handle this release.

HA CODE\#: 5

POINT OF CONTACT:

ACTION DUE:

REC CODE:

$$
\text { RESPONSIBLE ORG: }
$$

STATUS: OPEN

Status History:

12/21/94 Recommendation identification date 


\section{PRELIMINARY HAZARD ANALYSIS \\ ABC Chemicals \\ VCM Plant Conceptual Design}

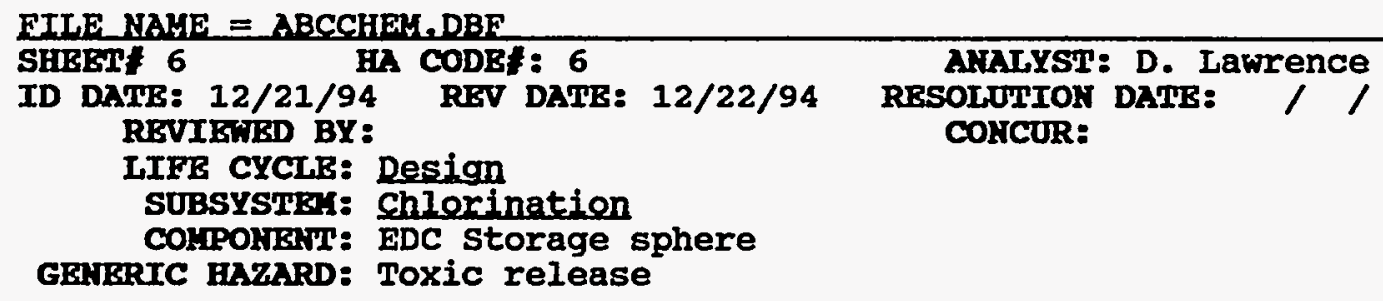

RISR ASSESSIIFIT:

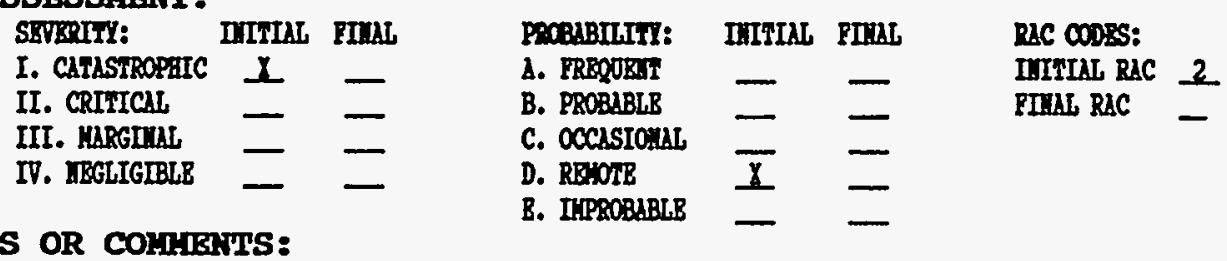

REHARRS OR COYRIBITS:

RECOMITENDATIONS TO MINIMIYE RISK:

1) Consider noving EDC sphere aray from river.

HA CODEF: 6

POINT OF CONTACT:

REC CODE:

STATUS: OPEN

Status History:

12/21/94 Recommendation identification date
ACTION DUE: / / RESPONSIBLE ORG: 


\section{PRELIMINARY HAZARD ANALYSIS \\ ABC Chemicals \\ vCM Plant Conceptual Design}

FILE NAYE $=$ ABCCHEM DBF

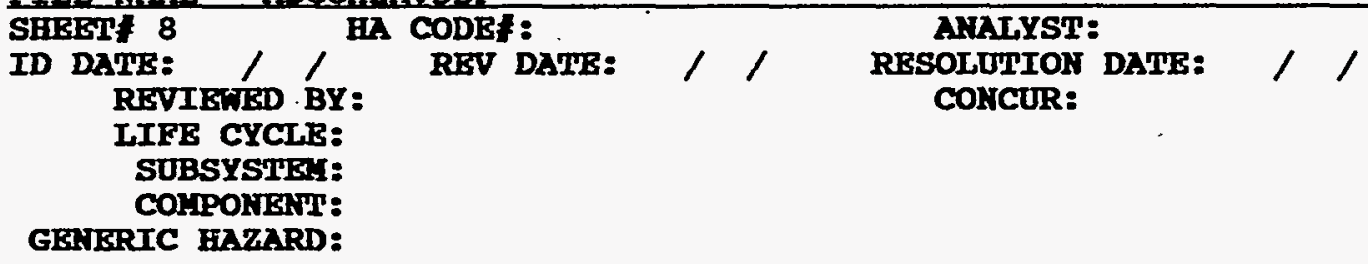

ANALYST:

RESOLITION DATE: / / COYCUR:

HAZARD SCEMARIO:

EFFECT:

RISR ASSBSSHIFIT:

SBTBRIT:

I. CATASTROPHIC

II. CRITICAL

III. HRRGIMLL

IV. MEGLIGIBLB

IIITIAL PIMAL

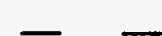

二

$=$

REFIARKS OR COYIEITS:

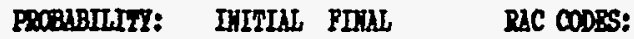

A. FREQUEMT - INITLLL RAC

B. PROBABLLE

c. occisionar - -

D. REMOIB

8. IIPROALBLIB 
Appendix C. Figures: CARMS

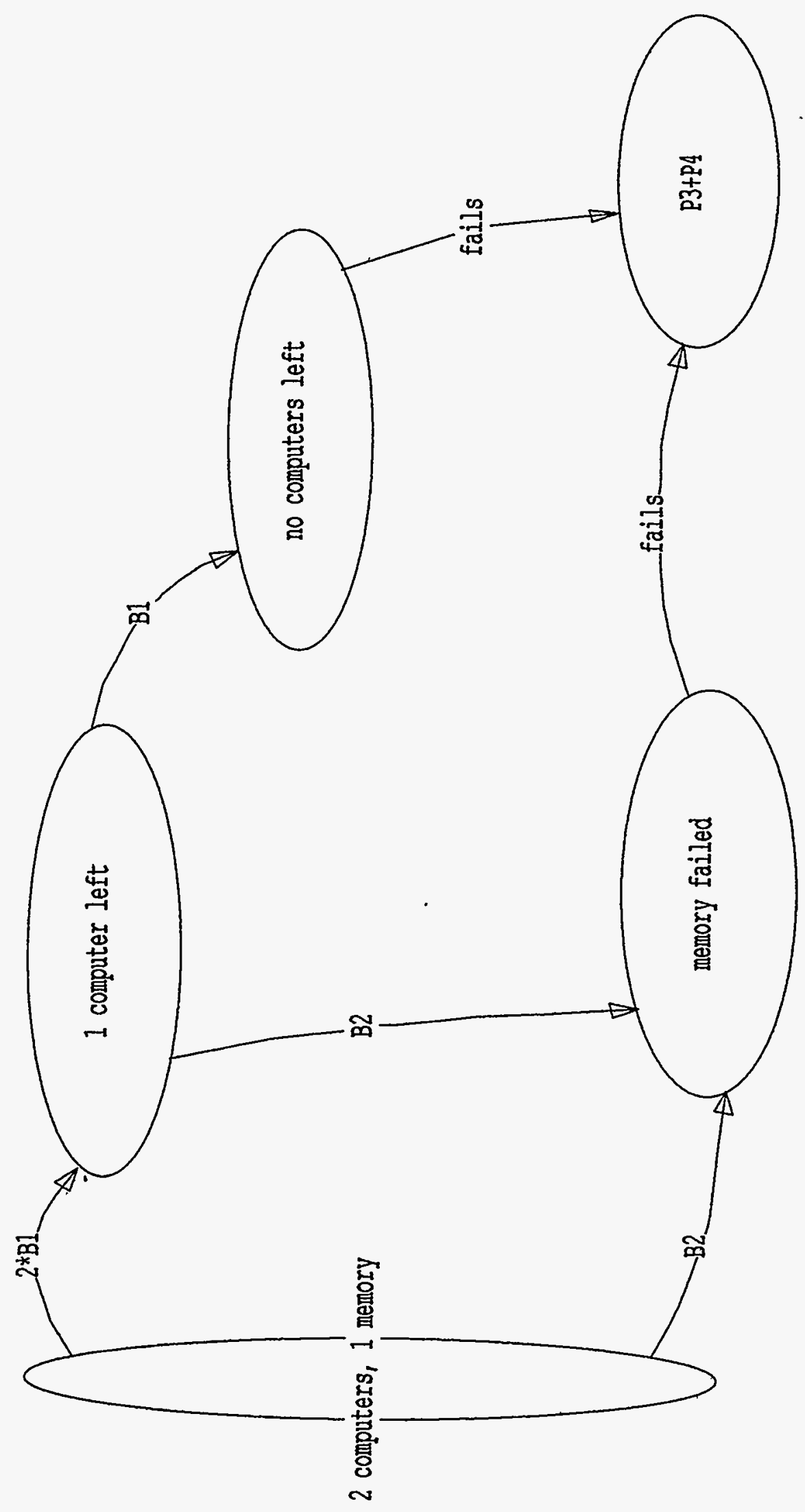




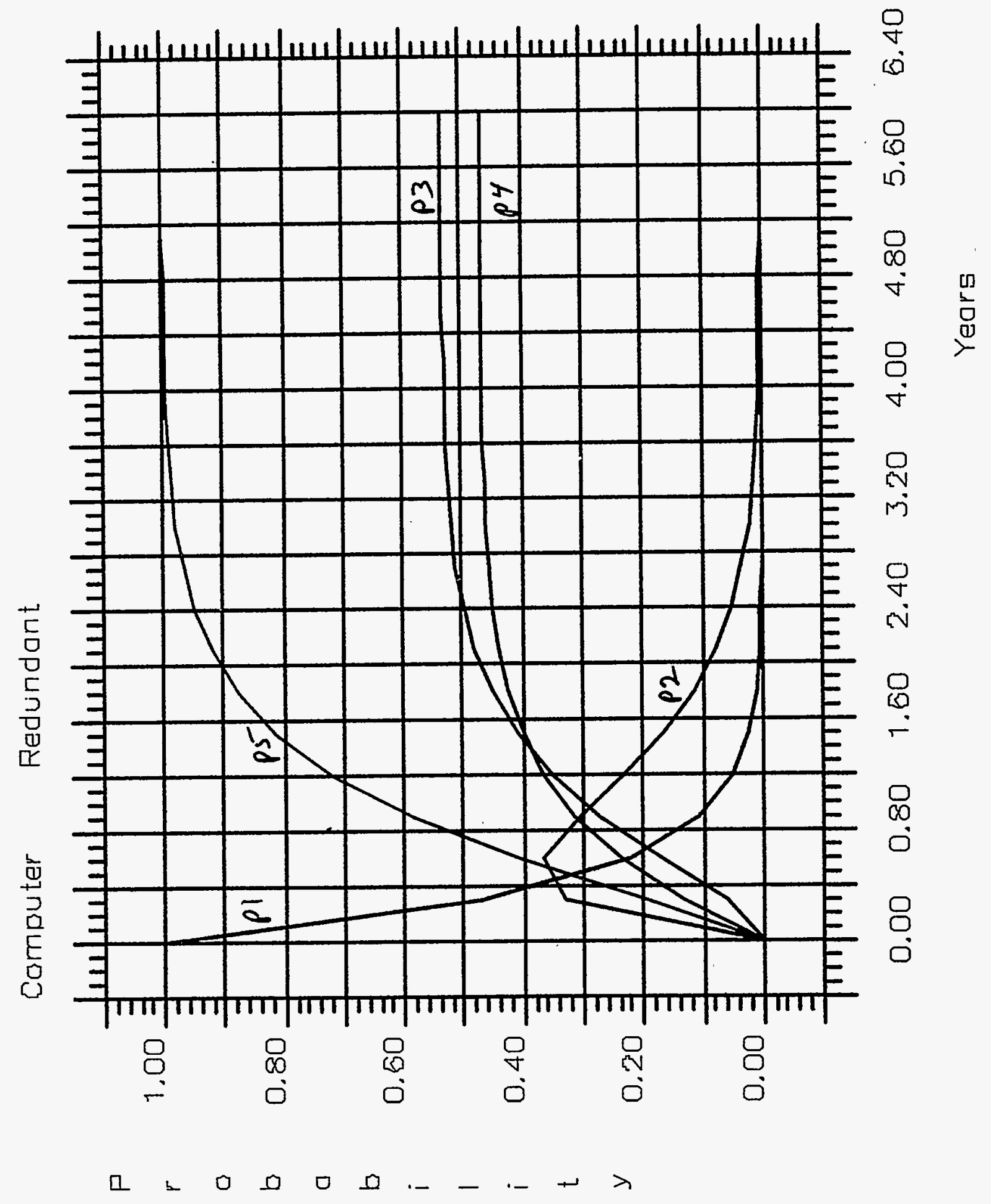


Appendix C. Figures: CARMS

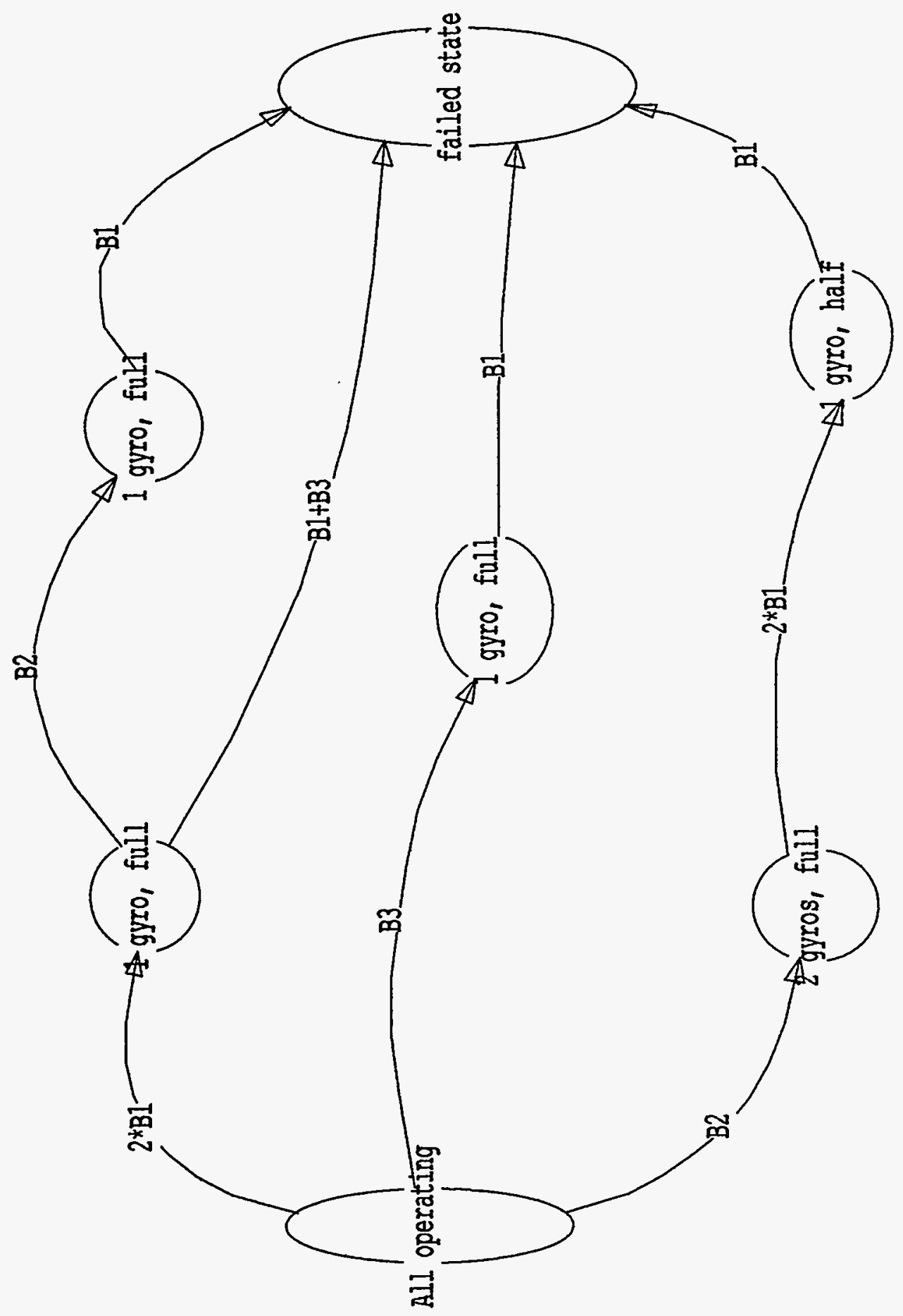




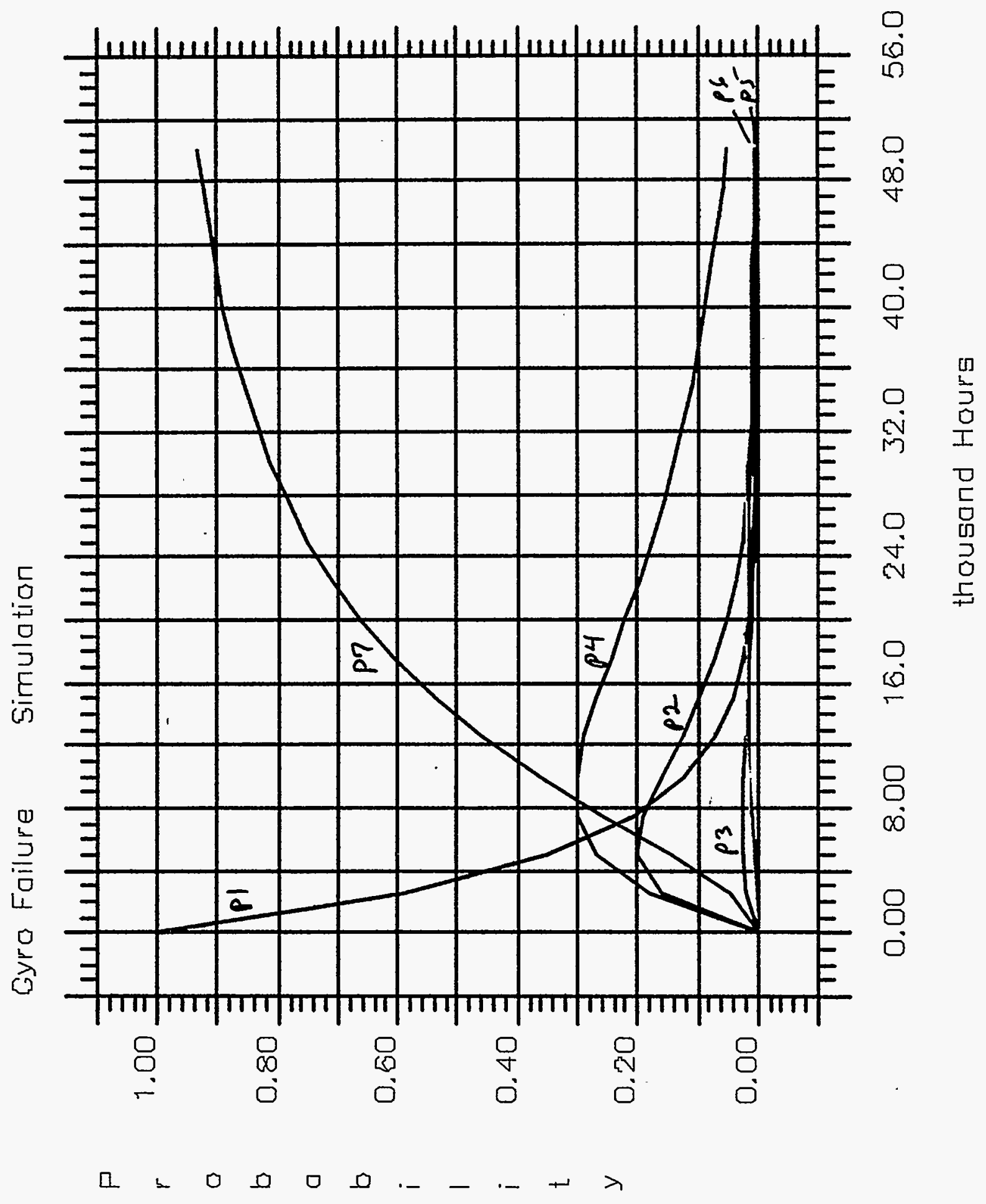




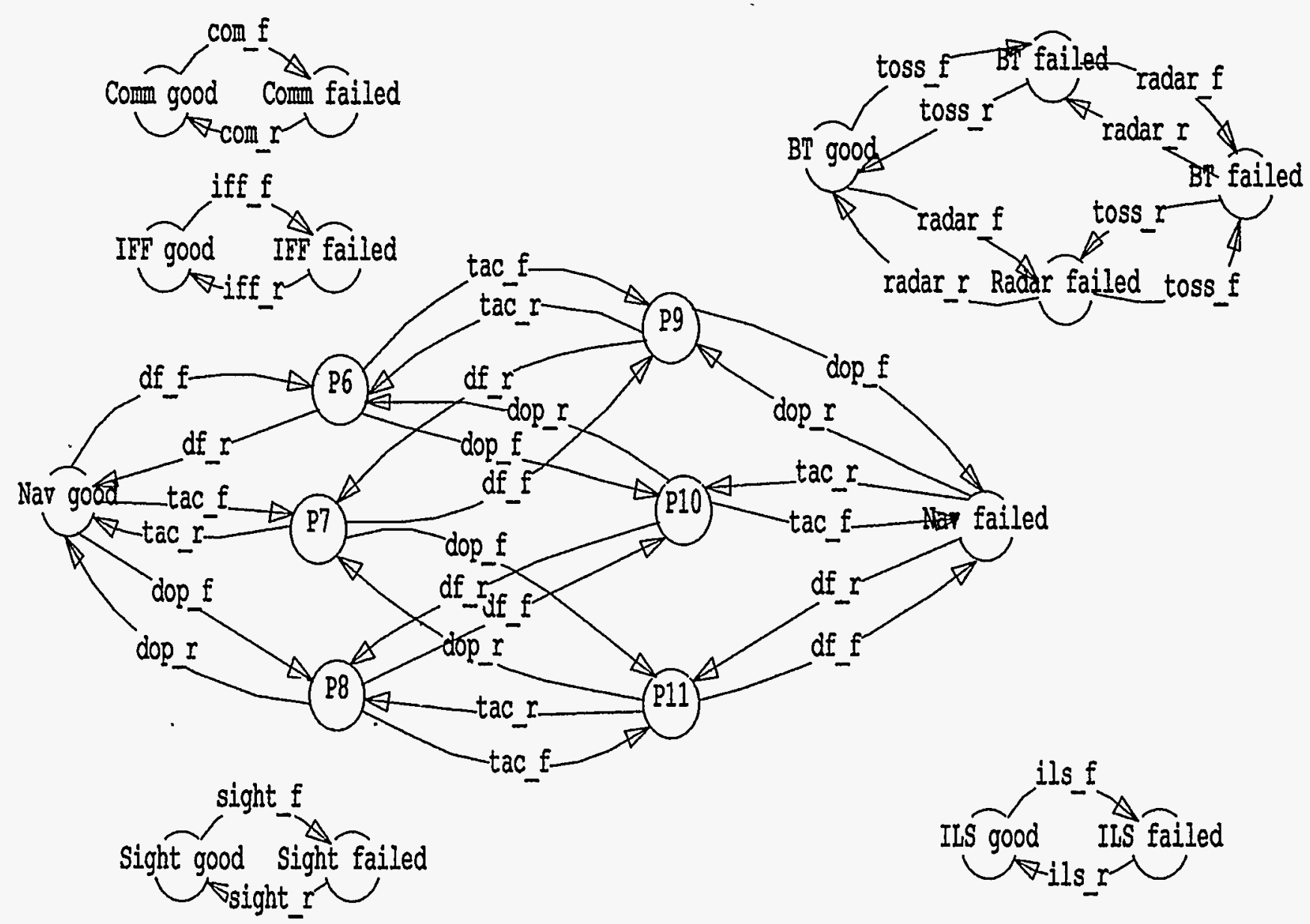

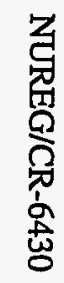
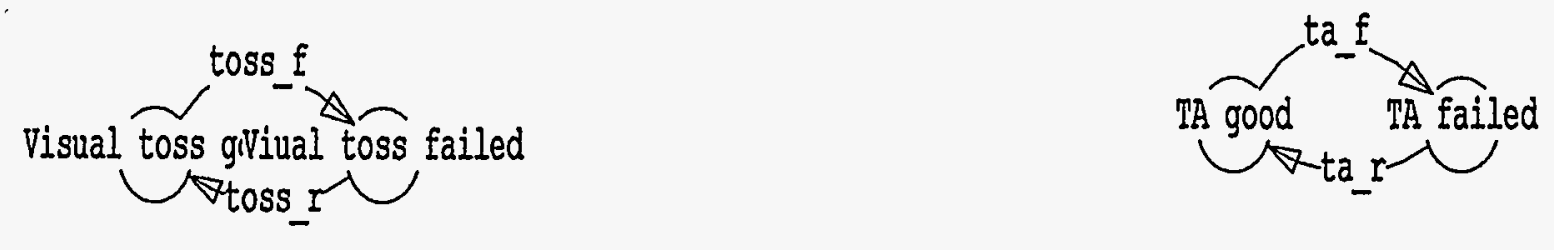


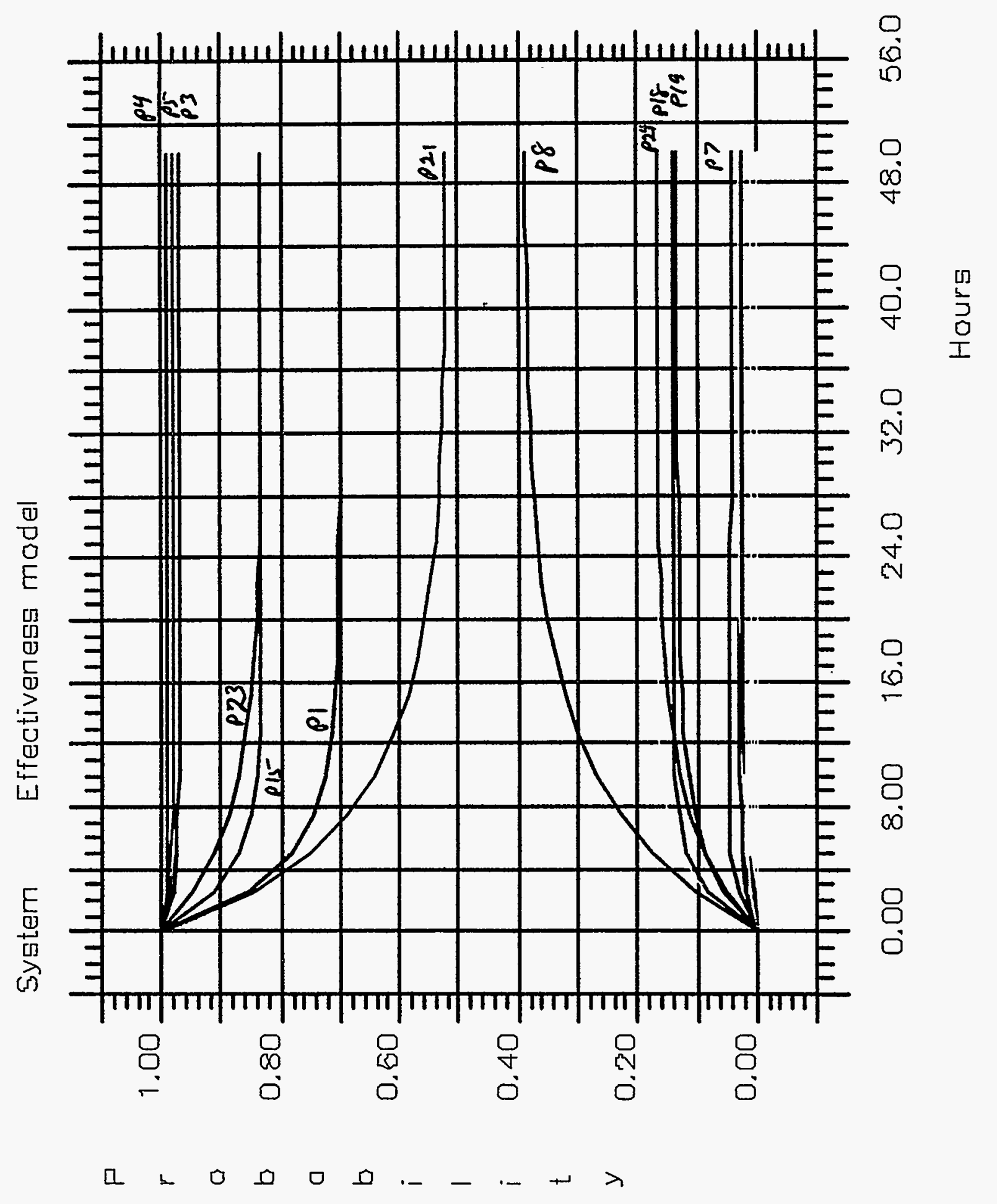




\section{Time between failures: fc_test.dat}

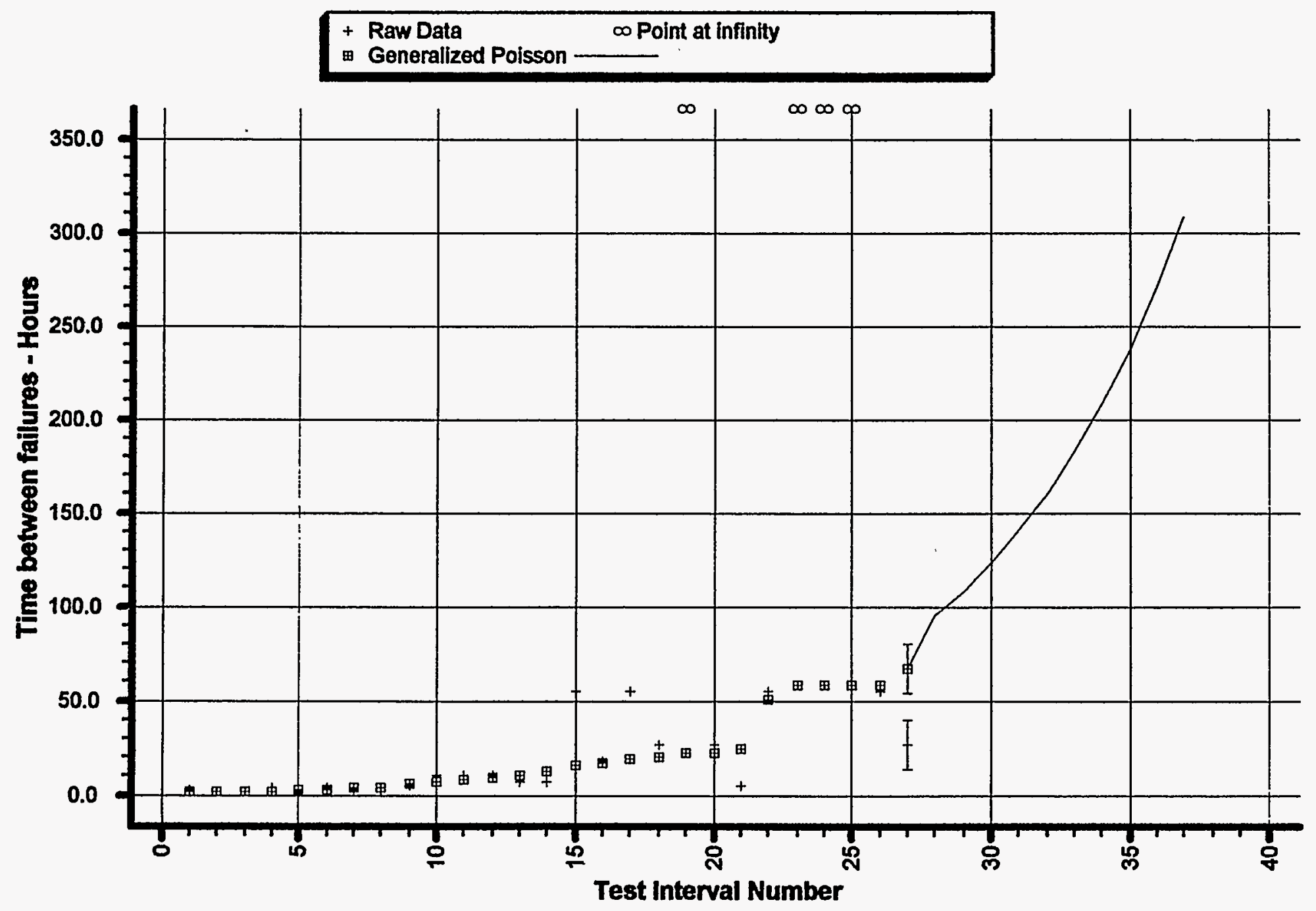




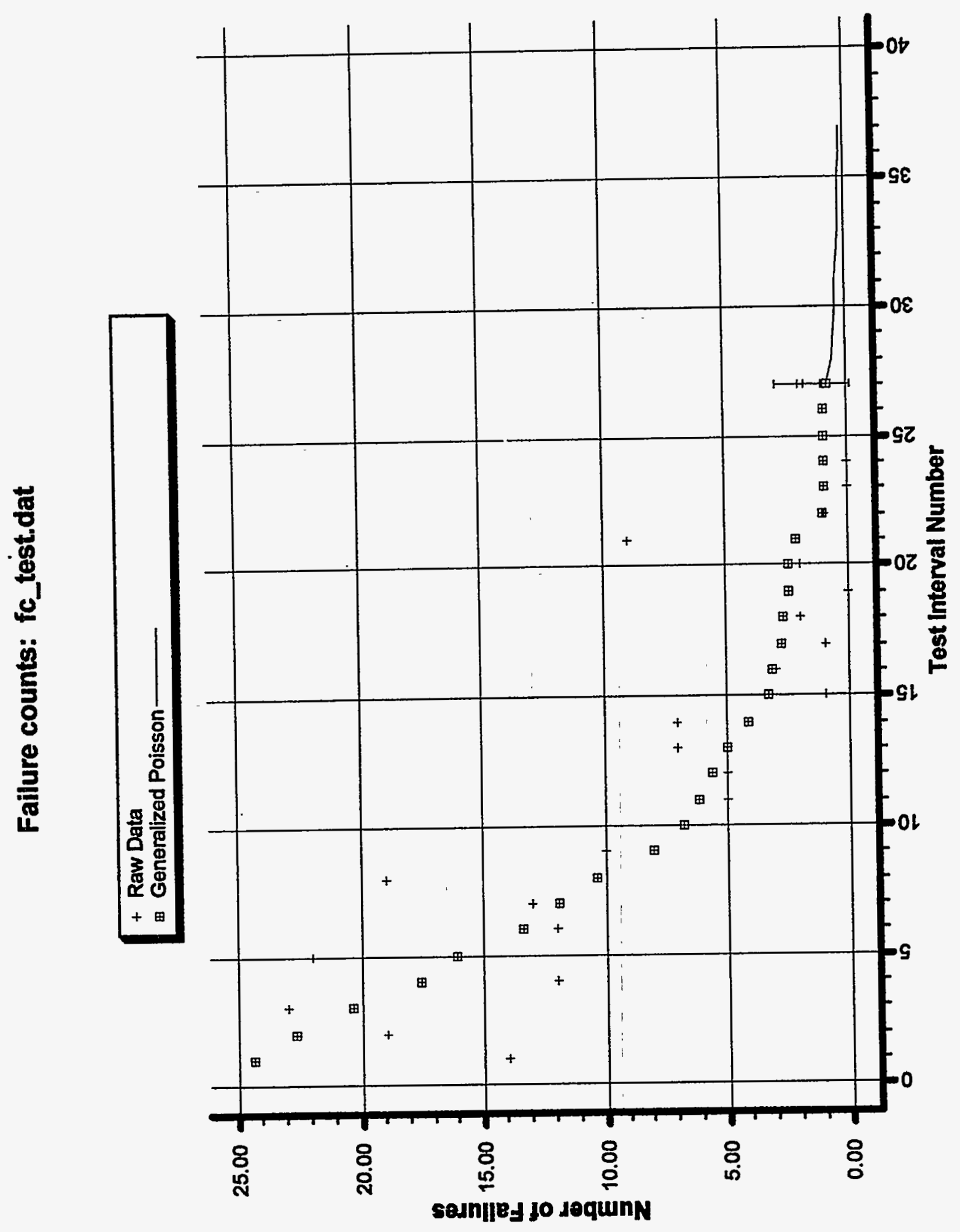




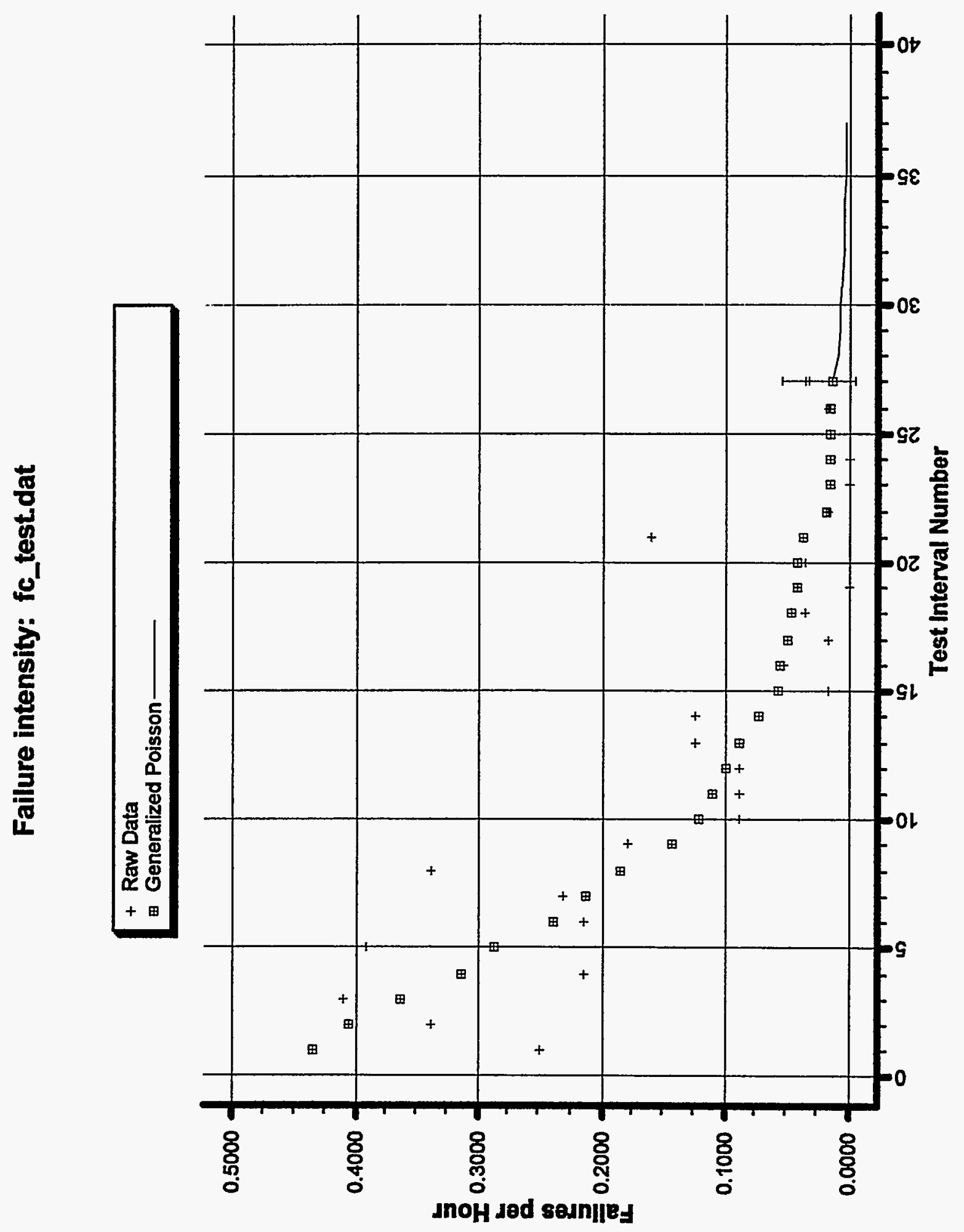




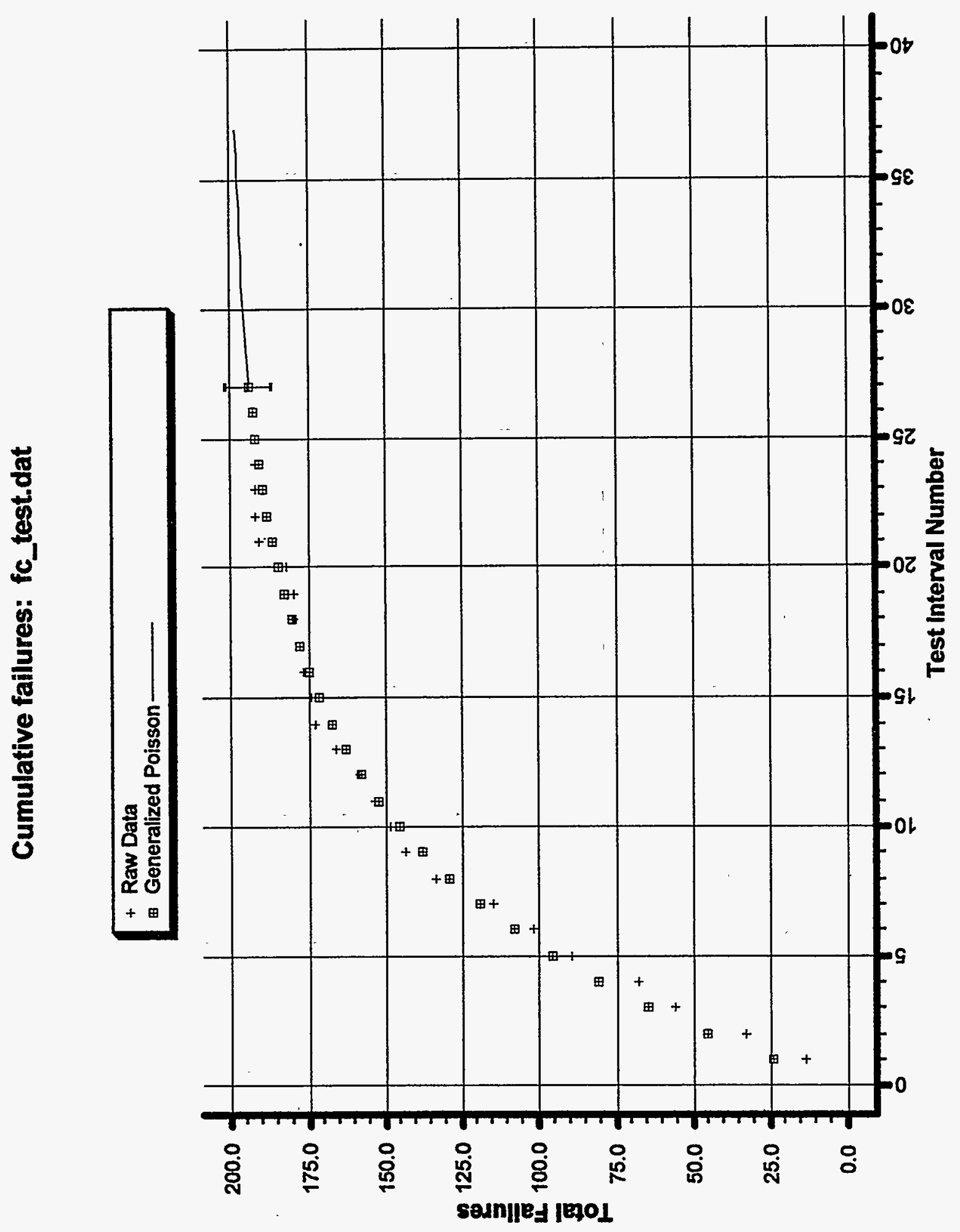




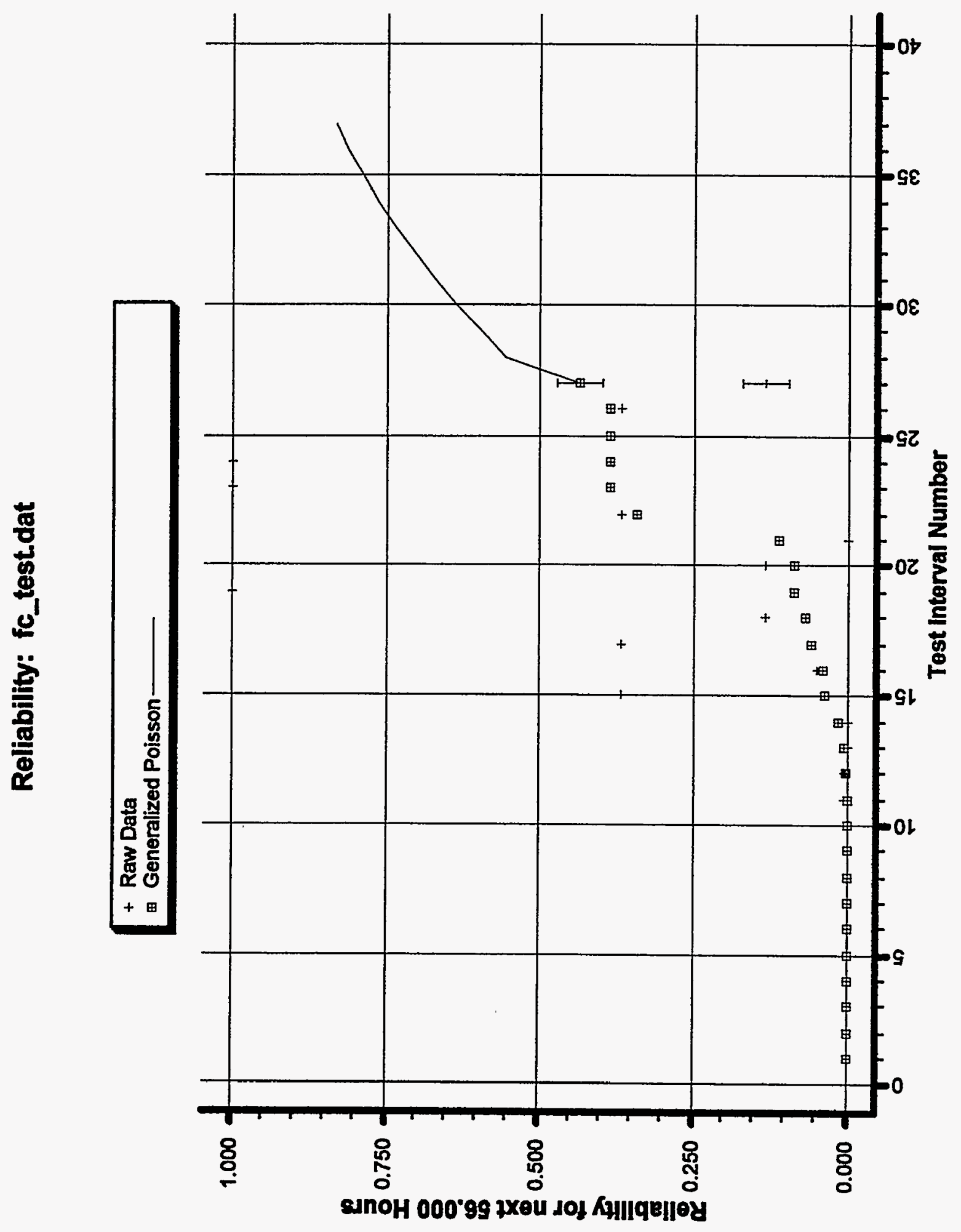




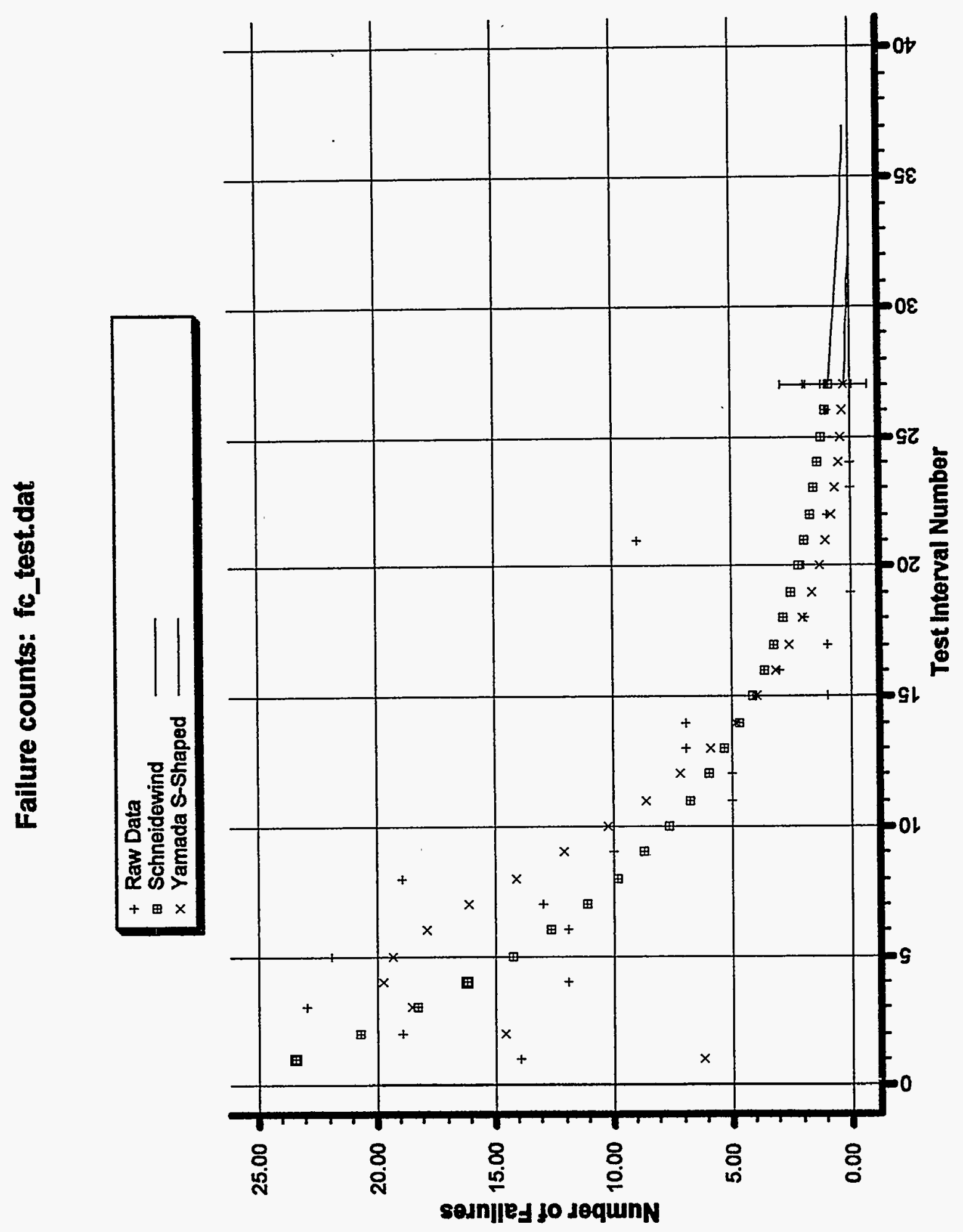




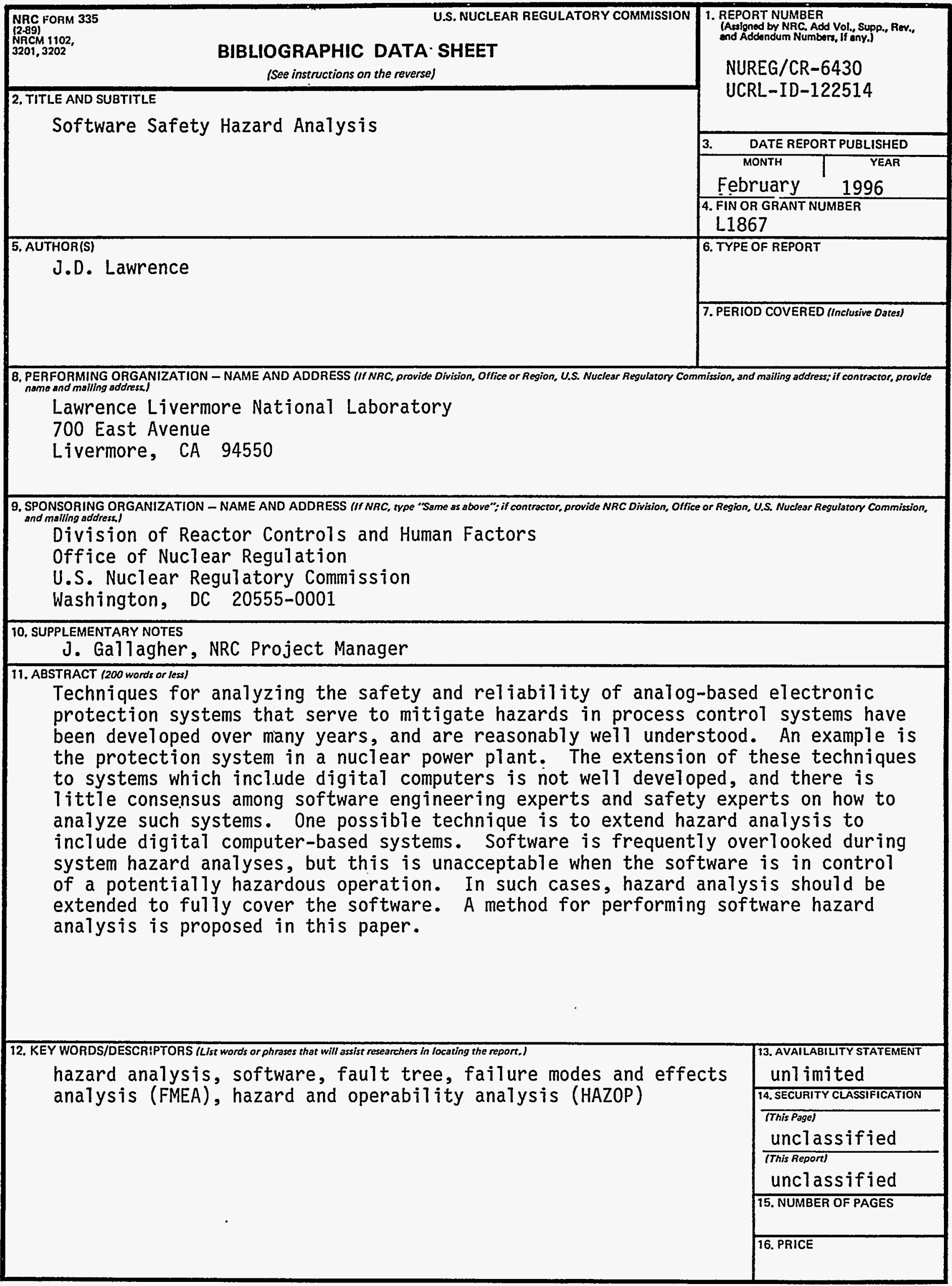






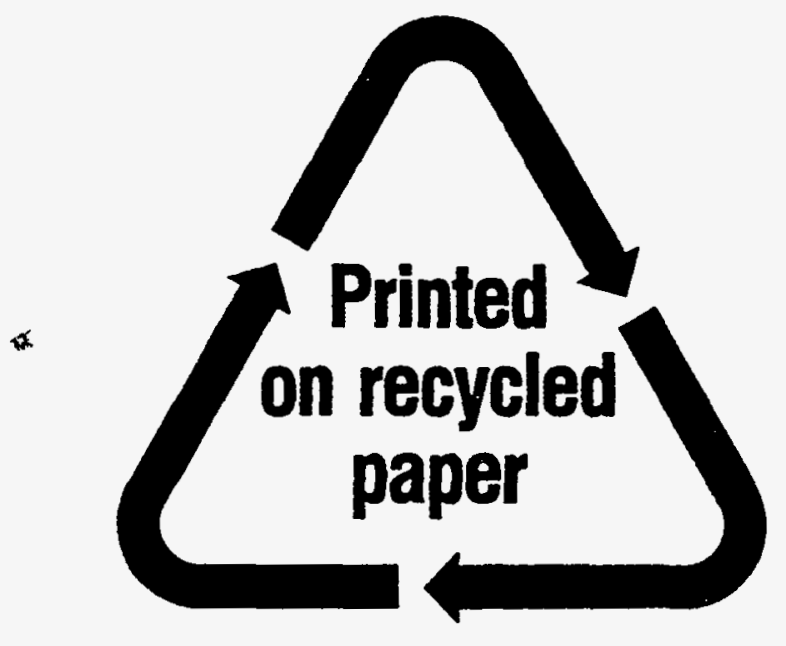

Federal Recycling Program 